\title{
Lessons for SUSY from the LHC after the first run
}

\author{
I. Melzer-Pellmann ${ }^{1, a}$, P. Pralavorio ${ }^{2, b}$ \\ ${ }^{1}$ DESY, Notkestr. 85, 22607 Hamburg, Germany \\ 2 CPPM, Univ. Aix-Marseille and CNRS/IN2P3, 163 Avenue de Luminy, Case 902, 13288 Marseille Cedex 09, France
}

Received: 10 February 2014 / Accepted: 17 February 2014 / Published online: 27 May 2014

(C) The Author(s) 2014. This article is published with open access at Springerlink.com

\begin{abstract}
A review of direct searches for new particles predicted by Supersymmetry (SUSY) after the first run of the LHC is proposed. This review is based on the results provided by the ATLAS and CMS experiments.
\end{abstract}

\section{Introduction}

Ernest Rutherford once said at the beginning of the twentieth century 'Theorists play games with their symbols while we discover truths about the Universe'. This was before the birth of the Bohr atom and quantum mechanics. At the beginning of the twenty-first century this sentence can probably be reverted, as the predictions of the theory are so successful that experimental discoveries of the last 40 years perfectly fit in the Standard Model (SM) theory framework. In this respect, the systematic exploration of the electroweak (EW) scale at the Large Hadron Collider (LHC) [1] could have represented an even greater confirmation of the theory predictions since most Beyond Standard Model (BSM) theories and, in particular, low-energy Supersymmetry (SUSY) expect new particles with masses close to the EW scale. As it will be discussed extensively in this article, this is clearly not the case. The direct searches conducted at the first LHC run by the general purpose experiments ATLAS [2] and CMS [3] did not reveal the presence of any new particle beyond that of the Standard Model. In contrary, the Standard Model is now fully established by the discovery of the Higgs boson $[4,5]$.

The article is based on currently published results obtained at a center-of-mass energy of $\sqrt{s}=8 \mathrm{TeV}$ for an integrated luminosity of $20 \mathrm{fb}^{-1}$ of LHC data. In case they are not available, previous results, obtained with $\sqrt{s}=7 \mathrm{TeV}$ and $5 \mathrm{fb}^{-1}$ of LHC data, are discussed. The most important analyses are based on $8 \mathrm{TeV}$ LHC data, either from ATLAS or CMS (or

\footnotetext{
a e-mail: isabell.melzer-pellmann@cern.ch

b e-mail: pascal.pralavorio@cern.ch
}

both). Therefore, even if the fully final result from Run 1 has not yet been published for all individual search channels, lessons for SUSY discussed here are not expected to change.

This article is organized as follows: First, a brief recap of the SUSY framework used for the LHC searches is proposed in Sect. 2. Then the experimental challenges faced by the ATLAS and CMS experiments in terms of object reconstruction and background modeling are explained in Sect. 3 . The limit setting procedure is also briefly summarized in this section. The discussion of the results is split in three different sections, representing the main avenues of the SUSY searches at LHC: gluino and squarks of first/second generation at the energy frontier in Sect. 4, third-generation squarks in Sect. 5, and electroweak SUSY in Sect. 6. All assume $R$ parity conservation and prompt decay of the SUSY particles. Section 7 is devoted to escape routes beyond $R$-parity conservation and prompt decays, as well as more exotic SUSY scenarios. Prospects with the coming runs of LHC and the conclusions are discussed in Sects. 8 and 9, respectively.

\section{SUSY framework for the search at LHC}

This section provides the reader with the minimum vocabulary and knowledge needed to understand the experimental results presented later in this article.

As discussed elsewhere in this review, SUSY [6,7] can be realized in many different ways. Even if the LHC cannot explore the full SUSY phase space, it can probe extensively the low-energy (or weak-scale) realization of $\mathrm{N}=1$ SUSY, called the Minimal Supersymmetric Standard Model (MSSM) [8]. This model predicts new particles, called sparticles, that are superpartners of each SM particle in the chiral multiplets, as shown in Fig. 1. A new quantum number, $R$ parity, is created and defined as $P_{R}=(-1)^{2 s+3 B+L}$, where $s$ is the spin, $B$ the baryon number and $L$ the lepton number. It is negative/positive for SUSY/SM particles. Therefore, the sparticles have the same quantum numbers as their SM 
Fig. 1 SUSY particles in MSSM [8]

\begin{tabular}{|c|c|c|c|c|}
\hline Names & Spin & $P_{R}$ & Gauge Eigenstates & Mass Eigenstates \\
\hline Higgs bosons & 0 & +1 & $H_{u}^{0} H_{d}^{0} H_{u}^{+} H_{d}^{-}$ & $h^{0} H^{0} A^{0} H^{ \pm}$ \\
\hline squarks & 0 & -1 & $\begin{array}{cccc}\tilde{u}_{L} & \widetilde{u}_{R} & \widetilde{d}_{L} & \tilde{d}_{R} \\
\tilde{s}_{L} & \widetilde{s}_{R} & \widetilde{c}_{L} & \widetilde{c}_{R} \\
\tilde{t}_{L} & \widetilde{t}_{R} & \widetilde{b}_{L} & \widetilde{b}_{R}\end{array}$ & $\begin{array}{c}\text { (same) } \\
(\text { same }) \\
\tilde{t}_{1} \tilde{t}_{2} \tilde{b}_{1} \tilde{b}_{2}\end{array}$ \\
\hline sleptons & 0 & -1 & $\begin{array}{ccc}\tilde{e}_{L} & \widetilde{e}_{R} & \widetilde{\nu}_{e} \\
\tilde{\mu}_{L} & \tilde{\mu}_{R} & \widetilde{\nu}_{\mu} \\
\tilde{\tau}_{L} & \widetilde{\tau}_{R} & \tilde{\nu}_{\tau} \\
\end{array}$ & $\begin{array}{c}\text { (same) } \\
\text { (same) } \\
\widetilde{\tau}_{1} \widetilde{\tau}_{2} \widetilde{\nu}_{\tau}\end{array}$ \\
\hline neutralinos & $1 / 2$ & -1 & $\begin{array}{llll}\widetilde{B}^{0} & \widetilde{W}^{0} & \tilde{H}_{u}^{0} & \tilde{H}_{d}^{0} \\
\end{array}$ & $\tilde{N}_{1} \tilde{N}_{2} \tilde{N}_{3} \tilde{N}_{4}$ \\
\hline charginos & $1 / 2$ & -1 & $\widetilde{W}^{ \pm} \widetilde{H}_{u}^{+} \widetilde{H}_{d}^{-}$ & $\widetilde{C}_{1}^{ \pm} \widetilde{C}_{2}^{ \pm}$ \\
\hline gluino & $1 / 2$ & -1 & $\widetilde{g}$ & (same) \\
\hline $\begin{array}{c}\text { goldstino } \\
\text { (gravitino) }\end{array}$ & $\begin{array}{c}1 / 2 \\
(3 / 2)\end{array}$ & -1 & $\widetilde{G}$ & (same) \\
\hline
\end{tabular}

partners, except for the spin which differs by half a unit, and $R$-parity. The spectrum is characterized by 25 elementary scalars and 10 elementary fermions without counting the SM particles.

For self-containment, we just recap the names and main characteristics of the new particles. To generate the masses of the up- and down-type fermions, the SM Higgs sector is extended by adding another $S U(2)_{L}$ complex doublet. Each doublet has a vacuum expectation value (vev) labeled $v_{u}$ and $v_{d}$, constrained by the SM Higgs vev, $v\left(v=\sqrt{v_{u}^{2}+v_{d}^{2}}\right)$. Their ratio is traditionally written $\tan \beta=v_{u} / v_{d}$. As a result, eight mass eigenstates exist after electroweak (EW) symmetry breaking: three neutral Higgs bosons $\left(\mathrm{h}^{0}\right.$, the one with the lightest mass, $\mathrm{H}^{0}$ and $\left.\mathrm{A}^{0}\right)$, two charged Higgs bosons $\left(\mathrm{H}^{ \pm}\right)$ and three Goldstone bosons $\left(\mathrm{G}^{0}, \mathrm{G}^{ \pm}\right)$, 'eaten' to give masses to the $\mathrm{Z}$ and $\mathrm{W}^{ \pm}$bosons. The Higgs boson discovered by ATLAS and CMS is assumed to be the lightest neutral Higgs of the MSSM $\left(\mathrm{h}^{0}\right)$, since it possesses similar properties to the $\mathrm{SM}$ one when $m_{\mathrm{A}^{0}}^{2} \gg m_{\mathrm{Z}}^{2}$ and $\tan \beta>1$ (also called the decoupling limit). ${ }^{1}$ The squarks $(\widetilde{\mathrm{q}})$ and sleptons $(\widetilde{1})$ are the spin-0 partners of the SM fermions. Similarly, winos $\left(\widetilde{\mathbf{W}}^{0}\right.$, $\left.\widetilde{\mathrm{W}}^{ \pm}\right)$, binos $\left(\widetilde{\mathrm{B}}^{0}\right)$, and higgsinos $\left(\widetilde{\mathrm{H}}_{u}^{0}, \widetilde{\mathrm{H}}_{d}^{0}, \widetilde{\mathrm{H}}_{u}^{+}, \widetilde{\mathrm{H}}_{d}^{-}\right)$are the spin-1/2 superpartners of the electroweak bosons. To complete the list, colored gluinos $(\widetilde{\mathrm{g}})$ and the gravitino $(\widetilde{\mathrm{G}})$ are the partners of the gluon and graviton. With this setup, the number of fermions and bosons is equalized and the Lightest SUSY Particle (LSP) is stable if $R$-parity is conserved.

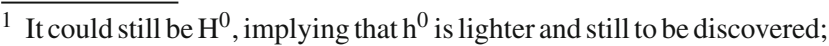
but this is presently disfavored by the data [9].
}

Note also that left- and right-handed fermions have two different SUSY partners $\widetilde{\mathrm{f}}_{L, R}$ that can mix in $\tilde{\mathrm{f}}_{1,2}$, provided the SM partner is heavy, like in the third generation. Similarly, the wino, bino, and higgsino, governed by the gauge eigenstate mass terms $M_{1}, M_{2}$, and $\mu$, mix to give four neutralinos $\left(\tilde{\chi}_{1,2,3,4}^{0}\right)$ and four charginos $\left(\tilde{\chi}_{1,2}^{ \pm}\right)$. To simplify the discussion of results in this sector, this article often considers one of the three typical scenarios, shown in Fig. 2. Each corresponds to a different $\widetilde{\chi}_{1}^{0}$ flavor: (a) bino-like, (b) wino-like or co-NLSP [10], and (c) higgsino-like. The last one is favored by naturalness arguments, as discussed in the next paragraph.

When choosing the parameters such that the hierarchy or naturalness problem is solved within the MSSM [11], stringent constraints appear on the masses of the new predicted particles, especially the ones which are most closely related to the Higgs, as can be seen in Fig. 3. Interestingly, there was no experimental sensitivity to this spectrum before the startup of the LHC. Moreover, by design, the LHC is perfectly suited to access this particular region of phase space, already with its first run at $\sqrt{s}=7-8 \mathrm{TeV}$. Searches for hints in this particular spectrum generally shape the analysis strategy at the LHC.

In the MSSM framework, three main theoretical unknowns influence the search direction: the LSP nature, the compression (or not) of the SUSY spectra, and the status of $R$-parity. For the first one, experimental constraints restrict the LSP to be the lightest neutralino $\left(\widetilde{\chi}_{1}^{0}\right)$ or the almost massless gravitino. In the latter case, the final states are increased compared to the former. The reason is that the Next-to-Lightest SUSY Particle (NLSP) which can be any of the SUSY particles (squark, gluino, slepton, chargino or neutralino) will 
(a)

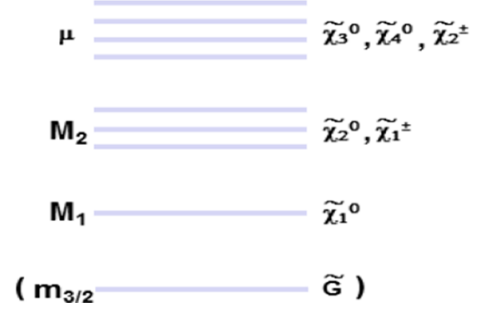

(b)

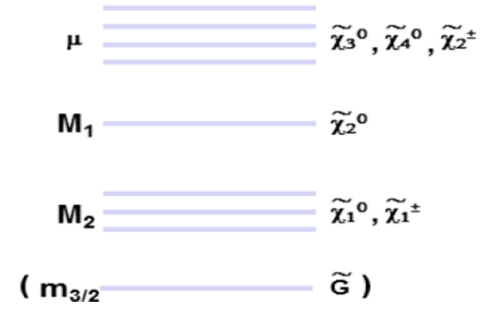

(c)

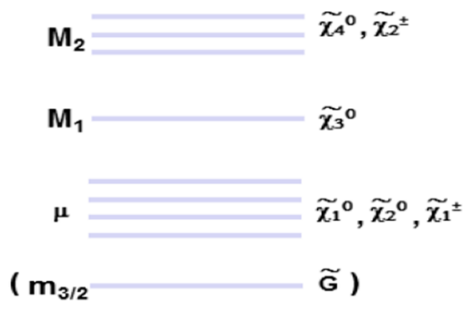

Fig. 2 Three possible EW SUSY mass spectra depending of the relative values of the $M_{1}, M_{2}$, and $\mu$ parameters

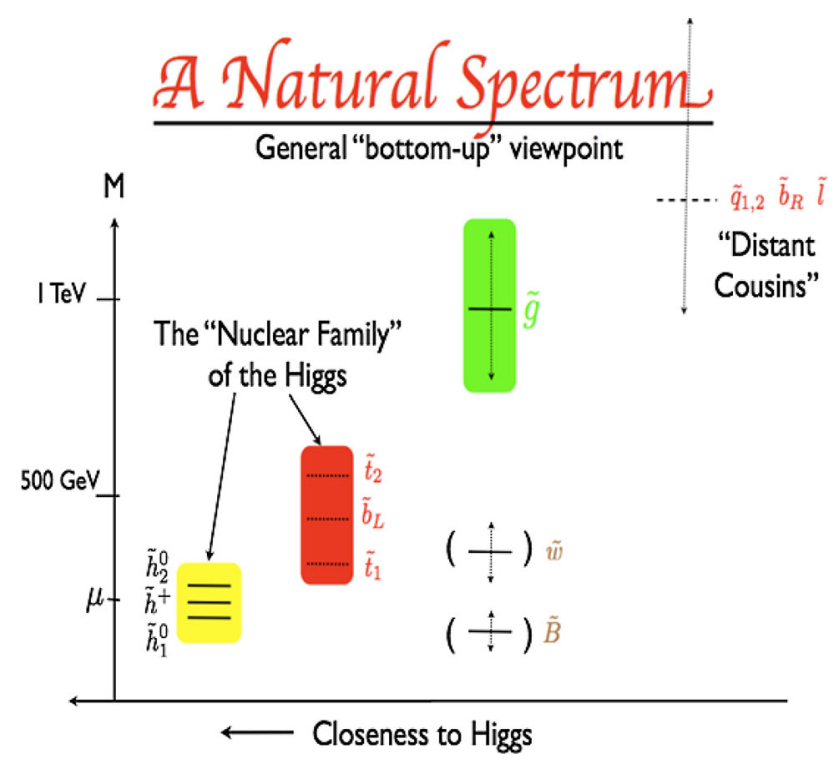

Fig. 3 Natural SUSY particle mass spectra giving less than $10 \%$ tuning [13]

decay to the gravitino and the SM partner of the NLSP [12]. ${ }^{2}$ The second MSSM theory unknown is the difference $(\Delta M)$ between the mass of the highest sparticle produced at the LHC $\left(M_{\text {SUSY }}\right)$ and the LSP $\left(M_{\text {LSP }}\right)$, resulting in compressed or open spectra, i.e. soft or hard objects in the final state. The third MSSM theory unknown is the status of $R$-parity. In a plain vanilla MSSM scenario $R$-parity is conserved (RPC), but it could well be violated (RPV) or even a continuous symmetry. It is important to mention that in the huge MSSM phase space long-lived particles decaying within the detector or even outside often exist. This situation could arise from the low mass difference between two sparticles in the spectrum, very weak coupling to the LSP, very small $R$-parity Yukawa couplings, etc.

\footnotetext{
2 The most 'natural' situation is that the NLSP is the $\widetilde{\chi}_{1}^{0}$ (Fig. 3), whose decay will depend on its flavor (bino-, wino- or higgsino-like of Fig. 2) and results in $\widetilde{\chi}_{1}^{0} \rightarrow \gamma \widetilde{\mathrm{G}}, \widetilde{\chi}_{1}^{0} \rightarrow \gamma / \mathrm{Z} \widetilde{\mathrm{G}}$ or $\widetilde{\chi}_{1}^{0} \rightarrow \mathrm{Z} / \mathrm{h}^{0} \widetilde{\mathrm{G}}$ decays, respectively. The relative proportion of $\gamma, \mathrm{Z}$ and $\mathrm{h}^{0}$ depends also on other parameters $\theta_{\mathrm{W}}$ or $\tan \beta$.
}

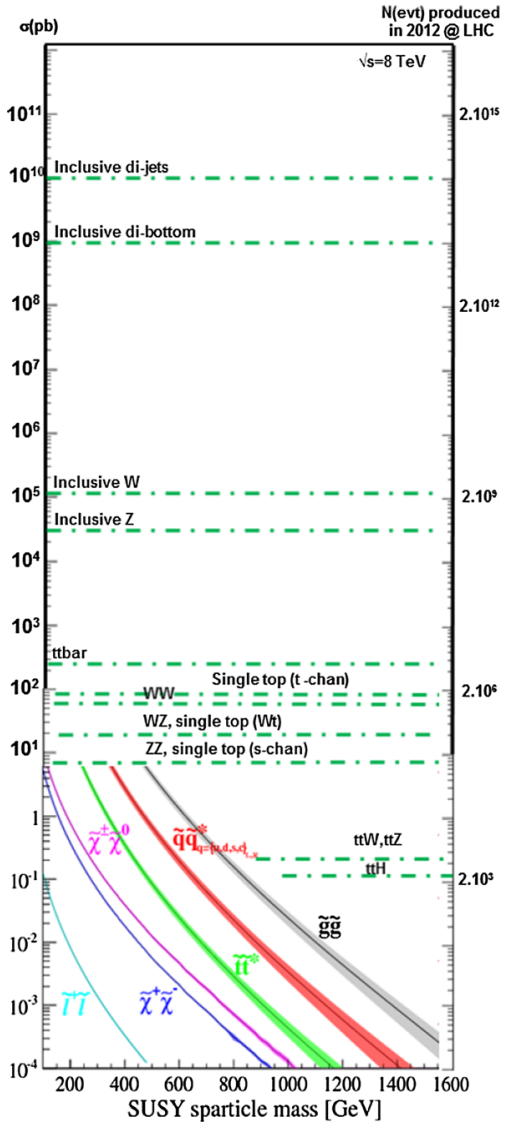

Fig. 4 Cross sections of several SUSY production channels [14], superimposed with Standard Model process at $\sqrt{s}=8 \mathrm{TeV}$. The righthanded axis indicates the number of events for $20 \mathrm{fb}^{-1}$

Typical SUSY cross sections of pair-produced sparticles at the LHC are given in Fig. 4-for two different sparticles mass degeneracy is assumed. ${ }^{3}$ Since each SM particle and its superpartner belong to the same multiplet, the sparticle decay generally involves the SM partner and the LSP. However, due to the high number of new particles many dif-

\footnotetext{
$\overline{3}$ Following an ATLAS-CMS agreement [14], all SUSY cross sections are calculated in the MSSM at NLO precision in the strong coupling constant, including the resummation of soft gluon emission at nextto-leading-logarithmic (NLO + NLL) accuracy, using PROSPINO and NLL-fast [15-19].
} 


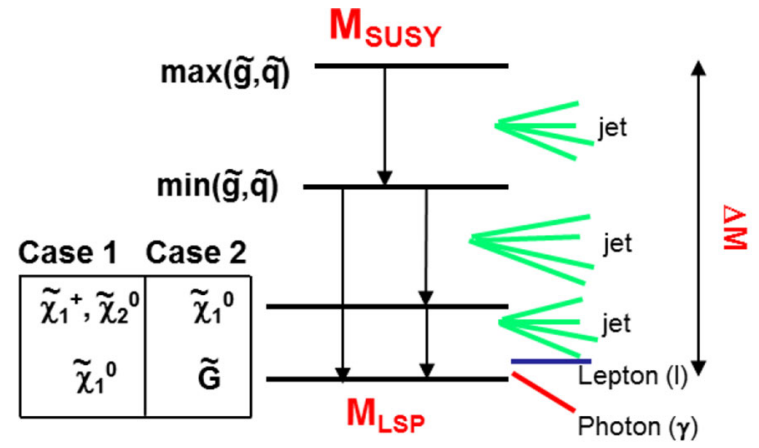

- Absolute value of vectorial sum: $\mathbf{E}_{\mathrm{T}}{ }^{\text {miss }} \sim \Delta \mathrm{M}$

- Scalar sum: $\mathbf{H}_{\mathrm{T}}=\Sigma \mathbf{p}_{\mathrm{T}}$ (jet) $\left[+\mathrm{p}_{\mathrm{T}}(\mathrm{l}, \gamma)\right] \sim \Delta \mathrm{M}$

$\cdot \mathbf{m}_{\mathrm{eff}}=\mathbf{E}_{\mathrm{T}}{ }^{\mathrm{miss}}+\mathrm{H}_{\mathrm{T}} \sim \mathbf{1 . 8}\left(\mathrm{M}_{\mathrm{SUSY}}{ }^{2}-\mathrm{M}_{\mathrm{LSP}}{ }^{2}\right) / \mathrm{M}_{\mathrm{SUSY}}[\mathrm{hep}-\mathrm{ph} / 0006276]$

Fig. 5 Typical decay of a colored SUSY particle at LHC. The two cases shown at the bottom of the SUSY spectrum correspond to the two considered LSP types

ferent decays are possible depending on the sparticle mass spectrum, generating long decay chains. Plain vanilla MSSM searches are therefore characterized by pair-produced particles, two LSPs escaping the detection and long decay chains involving jets and/or leptons and photons as depicted in Fig. 5. Note that for the gravitino LSP scenario, the mass difference with the NLSP is always sizeable in the natural spectrum, $\mathrm{O}(100) \mathrm{GeV}$, because the gravitino is almost massless. If the masses of all colored particles are too high to be produced at LHC, the production may be dominated by chargino-neutralino pair production, resulting in less complicated final states, generally containing several leptons. Finally, non-prompt sparticle decays can generate striking signatures as displayed in Fig. 6.

\section{Experimental challenges for SUSY searches at LHC}

This section is mainly addressed to non-experts in LHC analyses and analysers or theorists who want to understand better the many experimental facets of a SUSY analysis at LHC.

Discovering SUSY at the LHC is an extremely challenging task, even within the restricted framework of the MSSM. First, every corner of the parameter space needs to be cov- ered, including all possible decay channels which provide a high number of final states with different mixtures of reconstructed objects (photon, electron, muon, tau, jets, b-jets, missing transverse energy). Second, due to the presence of many scalars and weakly interacting particles, cross sections are generally extremely tiny with respect to the SM background (cf. Fig. 4). In the plain vanilla MSSM scenario, the few signal events are generally located in the tails of the kinematic distributions, requiring challenging trigger, powerful discriminating variables and accurate background modeling in a complicated region of the phase space. In other SUSY scenarios where $R$-parity is violated and/or non-prompt decays are possible, the experimental challenge generally shifts to taking the best performance of each subdetector to improve secondary vertex reconstruction, timing resolution, jet substructure reconstruction, lepton coverage, etc. Therefore, SUSY searches provide an excellent way to push the detector and analyser capabilities to their best.

This section is organized as follows. Experimental matters, i.e. LHC data, trigger and detector/object performance relevant for SUSY searches, are treated in Sect. 3.1. Commonly used discriminating variables for the design of the signal regions are then discussed in Sect. 3.2 and methods to estimate the remaining background in these signal regions are described in Sect. 3.3. Finally, the limit setting tools and SUSY models used for interpretations are briefly reviewed in Sects. 3.4 and 3.5, respectively.

\subsection{LHC data and detector performance}

After a brief reminder of the main characteristics of the LHC data (Sect. 3.1.1), ATLAS and CMS detectors (Sect. 3.1.2), the object and detector performance relevant to SUSY searches are discussed (Sects. 3.1.3 and 3.1.4).

\subsubsection{LHC data}

The LHC is a particle collider at CERN, 'probably the largest and the most complex machine ever constructed by humans' [20]. It is housed in a $27 \mathrm{~km}$ long tunnel $\sim 100 \mathrm{~m}$ underground and is ultimately designed to collide proton beams at a centerof-mass energy of up to $\sqrt{s}=14 \mathrm{TeV}$ at a rate of $40 \mathrm{MHz}$.
Fig. 6 Possible signatures from non-prompt sparticle decay

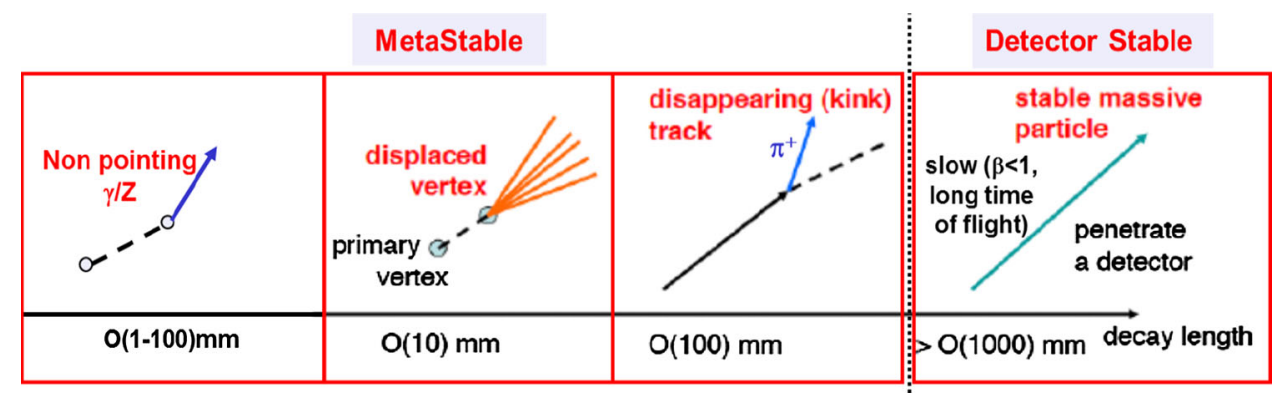


Fig. 7 LHC luminosity recorded by the CMS experiment

Fig. 8 Corresponding number of pile-up events as a function of time
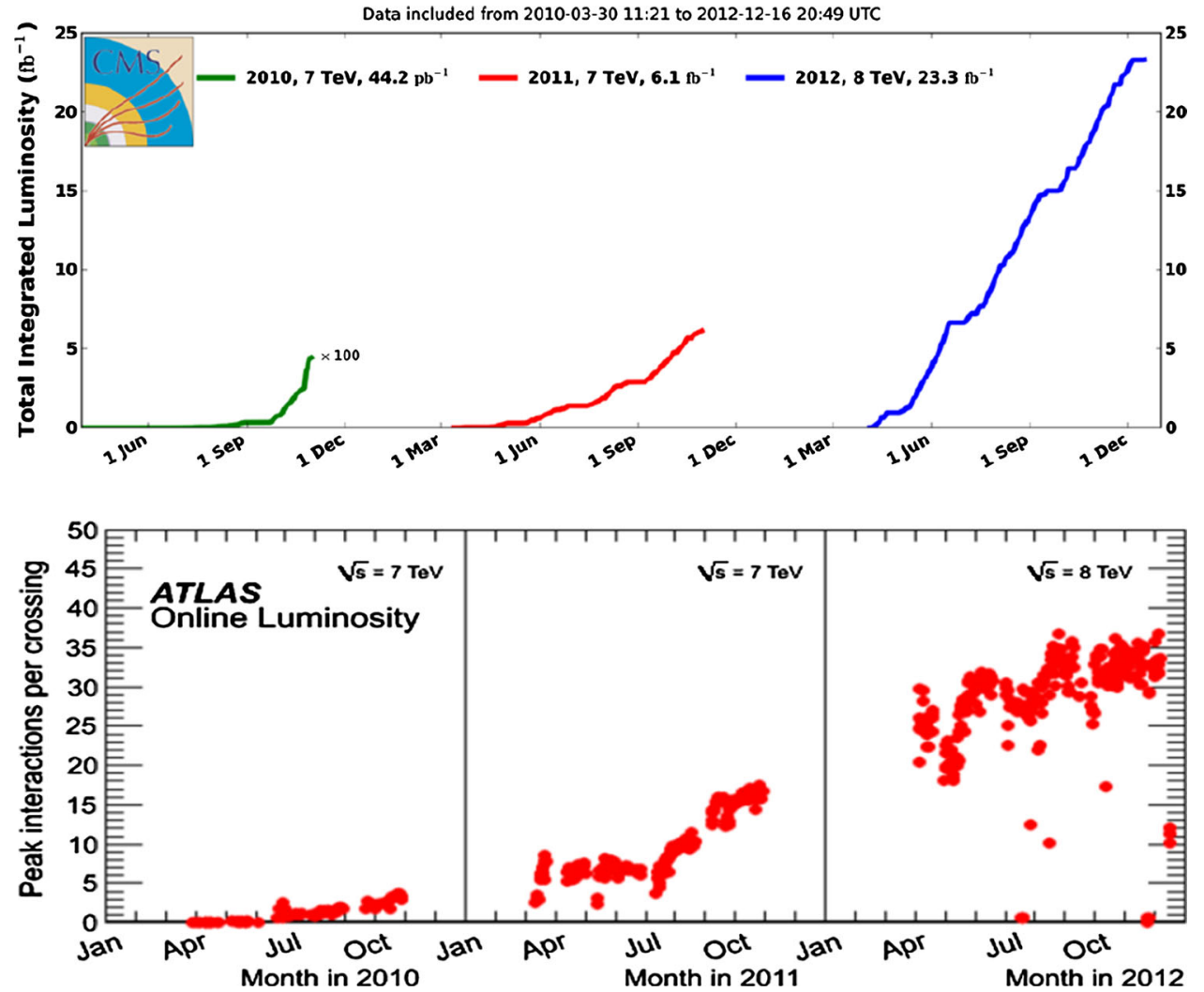

The first proton-proton run of the LHC (Run 1) lasted from March 2010 to December 2012 with $\sqrt{s}=7 \mathrm{TeV}$ and $8 \mathrm{TeV}$, and collisions every $50 \mathrm{~ns}$. It was extremely successful and provided more than $20 \mathrm{fb}^{-1}$ in 3 years, as shown in Fig. 7. Because of the increase of the proton density per bunch and the tuning of the beam optics, the number of interactions per beam crossing (pile-up) increased regularly during Run 1 to reach more than 30 at the end of 2012; see Fig. 8. This increases the complexity of the event reconstruction, as discussed later. For practical considerations, unless mentioned otherwise, searches presented in this article make use of the full $\sqrt{s}=8 \mathrm{TeV}$ dataset.

\subsubsection{ATLAS and CMS detectors}

Four large detectors have been built at the collision points. Among these, ATLAS and CMS are the two general-purpose experiments. Because of the huge complexity of the detectors that can cope with very high collision rate and high pile-up conditions, world-wide collaborations of a few thousands of physicists and engineers were set up, giving these projects the flavor of a modern cathedral, dedicated to science.

The interesting particles are produced over the full solid angle down to small polar angles $(\theta)$ with respect to the incoming beams (a fraction of a degree corresponding to pseudorapidities of $|\eta|$ up to 5 , where $\eta=-\ln [\tan (\theta / 2)]$ ) and in the full azimuth $0 \leq \phi \leq 2 \pi$. The transverse plane plays a special role for LHC analyses: the vectorial sum of all particle momenta produced by the collision is null and it is the bending plane in the central part. Therefore, selections generally rely on transverse momenta, labeled $p_{\mathrm{T}}$.

The two detectors are based on two different technologies for the central magnets that are used to bend the charged particle trajectories: CMS uses a 4-T superconducting solenoid magnet of $3 \mathrm{~m}$ radius, fully containing the trackers and the calorimeters, while ATLAS chose a smaller central solenoid ( $2 \mathrm{~T}$ and $1.2 \mathrm{~m}$ radius), complemented by outer toroids. These choices influence the design of all detector technologies [21].

Inner tracking systems measure the momentum of charged particles, which are bent by the magnetic field. To achieve this, ATLAS and CMS have designed tracker systems providing a similar geometrical coverage (over $|\eta|<2.4-2.5$ ). They are based on the same silicon detector technology near the interaction vertex, i.e. below $50 \mathrm{~cm}$, with a silicon pixel and strip tracker, providing around 10 precision points per track. However, they differ considerably at larger radii: ATLAS uses straw-tube detectors (TRT), allowing 35 extra measurements per track in the bending plane for $|\eta|<2.0$, with drift-time information for momentum measurements and pattern recognition, while CMS extends the siliconstrip technology up to a radius of $\sim 110 \mathrm{~cm}$. Stand-alone tracker performance is generally better for CMS because of the higher magnetic field. 
The electromagnetic (EM) calorimeter is the key system for measuring the kinematics of electrons and photons. CMS chose $\sim 75000$ scintillating $\mathrm{PbWO}_{4}$ crystals with an excellent energy resolution but extremely low light yield, while ATLAS built a granular lead/liquid argon sampling calorimeter with $\sim 200 \mathrm{k}$ channels. This last technology is robust and well known, with poorer energy resolution at low energy, but comparable to CMS in the $0.1-1 \mathrm{TeV}$ energy range.

Key parameters for the hadronic calorimeters are the coverage up to $|\eta|<5$ for both ATLAS and CMS, the depth in interaction length $(\lambda \sim 10)$ and the sampling fraction, three times better in ATLAS than in CMS [21].

Finally, the muon spectrometers are also quite different: in ATLAS they can provide an independent and high-accuracy measurement of muons over $|\eta|<2.7$ coverage, whereas CMS relies on a combined measurement of muon chambers and inner tracker up to $|\eta|<2.4$.

The ability of ATLAS and CMS to use more than $90 \%$ of the high-quality data delivered by the LHC for physics analyses demonstrates the excellent functioning of both experiments.

\subsubsection{Object reconstruction performance relevant to SUSY searches}

Because of different detector concepts, the final-state reconstruction differs quite a lot between ATLAS and CMS.

In ATLAS, electrons, photons, and jets are seeded by calorimeter clusters. Electrons and photons are eventually combined with the tracker information. In CMS, all finalstate particles are reconstructed with the particle-flow method [22], generally seeded in the inner detector and further combined with the information from all sub-detectors.

Jets are reconstructed using the anti-kt jet clustering algorithm [23,24] with a distance parameter of 0.4 and 0.5 for ATLAS and CMS, respectively. After calibration, pile-up corrections and cleaning, jets are generally considered only above $p_{\mathrm{T}}>20 \mathrm{GeV}$. The jet energy scale uncertainty (and the jet energy resolution to a lesser extent) is generally the dominant systematic uncertainty for $R$-parity conserving strong production. It is lower than $2 \%$ for $p_{\mathrm{T}}>100 \mathrm{GeV}$, degrading to $4 \%$ for jets with $p_{\mathrm{T}}=20 \mathrm{GeV}[25,26]$.

Identifying b-jets from light-quark and gluon jets is crucial for all third-generation searches and is possible thanks to secondary vertex information provided by the tracker. Dedicated algorithms are used by ATLAS [27] and CMS [28]. Typically with a 60-70 \% b-jet identification efficiency, a light-quark jet rejection between 100 and 1000 is obtained depending on $p_{\mathrm{T}}$ and $\eta$. Similarly, hadronically decaying taus can be separated from light-quark jets with $\mathrm{O}(10)$ less rejection power than for a b-jet assuming a $60 \%$ tau identification efficiency [29].
Leptons are key ingredients for SUSY searches targeting compressed spectra, EW production, and/or RPV final states. Due to trigger requirements, the leading lepton has to be generally above $20-25 \mathrm{GeV}$ in $p_{\mathrm{T}}$, but it is possible to lower the $p_{\mathrm{T}}$ down to 6-7 GeV in analyses considering multi-leptons. Further separation from jets is obtained by requiring the leptons to be isolated in the calorimeters and/or the tracker.

A crucial variable for SUSY searches at the LHC is the magnitude of the missing transverse momentum vector $\left(E_{\mathrm{T}}^{\mathrm{miss}}\right)$. In ATLAS, it is based on the vector sum of transverse momenta of jets, leptons and all calorimeter clusters not associated to such objects (within $|\eta|<5$ ). In CMS, it is based on all particles reconstructed by the particleflow method which compensate for the lower calorimeter jet energy resolution. Because of the hadronic environment, fake $E_{\mathrm{T}}^{\mathrm{miss}}$ can arise from jet mismeasurements which can be efficiently removed by rejecting events where a high-energetic jet (or lepton) and $\mathbf{E}_{\mathrm{T}}^{\text {miss }}$ are close-by (with a relative angle of $\left.\Delta \phi\left(j, E_{\mathrm{T}}^{\text {miss }}\right)<0.4\right)$. Detector malfunctions and poorly instrumented regions can cause high $E_{\mathrm{T}}^{\text {miss }}$ as well. In some lepton-veto analyses, $H_{\mathrm{T}}^{\text {miss }}$ is considered, i.e. the vectorial sum of jets above few tens of $\mathrm{GeV}$ in $p_{\mathrm{T}}$, to decrease the sensitivity to low-energy jets coming from pile-up.

Obviously the very first experimental challenge at the LHC is the trigger. In Run 1, ATLAS and CMS concentrated their efforts on single triggers $\left(E_{\mathrm{T}}^{\mathrm{miss}}\right.$, multi-jets, electrons, and muons), but they also allocated a part of the bandwidth to combined triggers (di- and multi-leptons, jets $+E_{\mathrm{T}}^{\text {miss }}$, several central jets, leptons in combination with large hadronic energy, etc.) which are more analysis-specific.

\subsubsection{Detector performance relevant to SUSY searches}

Performance of specific sub-detectors are crucial to detect non-prompt sparticle decays or heavy stable charged particles which are slowly moving $(\beta=v / c<0.9)$.

In this respect, the tracker provides a lot of relevant information: (i) the ionization energy loss $(\mathrm{d} E / \mathrm{d} x)$ measured in the silicon detectors, significantly higher for low $\beta$ than for minimum ionizing particles, (ii) characteristics of displaced vertices via dedicated algorithms, and (iii) for ATLAS a continuous outer tracker, the TRT, to identify late decays. Note that RPC searches generally veto long-lived particles decaying in the tracker by imposing impact parameter requirements to reject cosmic muons.

Low- $\beta$ particles or particles coming from long-lived particle decays in the tracker will arrive late in the calorimeter. The excellent timing resolution of the ATLAS and CMS EM calorimeters, around $0.3-0.4$ ns per cell, is a very powerful tool to discriminate against SM particles. In the case of ATLAS, stand-alone pointing capability can also be used. Similarly, the excellent control of the calorimeter noise yields sensitivity to late-decaying particles trapped in the detector. 
Finally, the time-of-flight measured in the muon spectrometer can be exploited as well. Stand-alone resolution of the order of $5 \%$ or better can be achieved over the whole $\eta$ range of the spectrometer. It can be ultimately improved by combining information with the calorimeter; see for example [30].

\subsection{Discriminating variables}

In RPC searches, the main background for SUSY signals is generally caused by processes involving particles with masses close to the weak scale (W, Z, H, top), or QCD multijet production with real or fake $E_{\mathrm{T}}^{\text {miss }}$; see Fig. 4. These processes can have cross sections that are up to ten orders of magnitude higher than the SUSY signal. Therefore, finding SUSY at the LHC requires to design signal regions (SR) by exploiting at best the main characteristics of the decay cascade: the presence of two stable LSPs, whose momentum is directly proportional to $E_{\mathrm{T}}^{\mathrm{miss}}$, and/or long decay chains involving jets, i.e. large calorimeter activity in the event. The latter is efficiently measured by the scalar sum, $H_{\mathrm{T}}$, of the transverse energy of reconstructed objects (for some analyses, only a part of the objects can also be considered). Rectangular cuts can then be applied on $H_{\mathrm{T}}$ and $E_{\mathrm{T}}^{\text {miss }}$ (or $H_{\mathrm{T}}^{\text {miss }}$ ).

Sensitivity can be improved by taking advantage of the correlation between $H_{\mathrm{T}}$ and $E_{\mathrm{T}}^{\text {miss }}$ and by computing $m_{\mathrm{eff}}=$ $H_{\mathrm{T}}+E_{\mathrm{T}}^{\text {miss }}$, called the effective mass [31]. This is illustrated in Fig. 9. The other advantage is that $m_{\text {eff }}$ can be linked to characteristic SUSY parameters like $M_{\text {SUSY, the }}$ mass of the highest colored object, $M_{\mathrm{LSP}}$, the LSP mass, and their mass difference $\Delta M$; see Fig. 5. Typically $m_{\mathrm{eff}}$ will

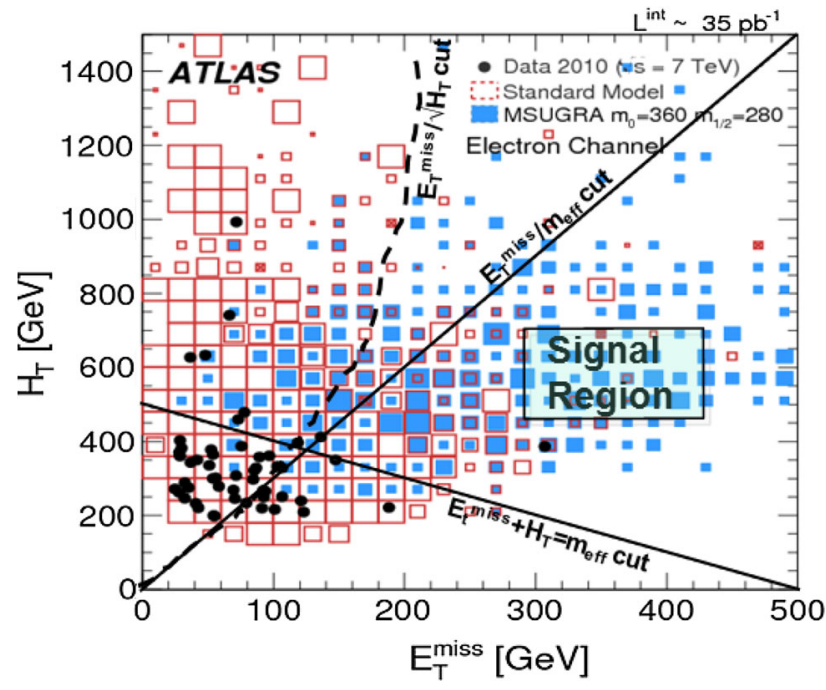

Fig. 9 Illustration of the variables used in a standard SUSY search for strong production at $\mathrm{LHC}$ with one-lepton, three jets and $E_{\mathrm{T}}^{\mathrm{miss}}$ final state [33]. For completeness the variable $E_{\mathrm{T}}^{\text {miss }} / \sqrt{H_{\mathrm{T}}}$ is also represented in this plane peak at $1.8\left(M_{\mathrm{SUSY}}^{2}-M_{\mathrm{LSP}}^{2}\right) / M_{\mathrm{SUSY}}$ [32]. For open spectra $\left(\Delta M=M_{\mathrm{SUSY}}-M_{\mathrm{LSP}}>\mathrm{O}(500) \mathrm{GeV}\right)$, this value is well above the SM background which has no correlation between $E_{\mathrm{T}}^{\mathrm{miss}}$ and $H_{\mathrm{T}}$, and therefore peaks at lower values. However, for compressed spectra $(\Delta M<500 \mathrm{GeV}), m_{\text {eff }}$ loses its separation power and selection requirements have to be relaxed, or other discriminant variables have to be used.

An alternative approach, which can also served as a crosscheck, is to take advantage of the kinematic distribution of the decay of two heavy sparticles (typically gluinos or squarks around $1 \mathrm{TeV}$ ). By grouping the reconstructed objects in two hemispheres, two mega-jets can be formed and kinematic properties used to distinguish signal from SM background. Two common variables are $\alpha_{\mathrm{T}}$ [34], the ratio of the $p_{\mathrm{T}}$ of the second hardest jet and the invariant mass formed from the two hardest jets, and Razor [35]. In the former case, SM dijet events are back-to-back and trail off at $\alpha_{\mathrm{T}}=0.5$, whereas a SUSY signal can be asymmetric (causing $\alpha_{\mathrm{T}}>0.5$ ) because of the presence of the LSP in the decay. For Razor, the idea is to use the transverse and longitudinal information to reconstruct the mass $M_{R}$ of the two mega-jets in the rest frame of the two-jet system ( $R$-frame). For signal with open spectra $M_{R}$ will peak at $M_{\mathrm{SUSY}}$ and at $m_{\mathrm{t}}$ or $m_{\mathrm{W}}$ for $\mathrm{t}_{\mathrm{t}}$ and $\mathrm{WW}$ events. Other quantities related to the $R$-frame (transverse mass, Lorentz boost) can be used to increase the discrimination against the background.

If the presence of lepton(s) is required, several types of invariant transverse masses could be considered as discriminant variables. The simplest case corresponds to the single lepton channel. There, the leptonic W-boson background, either coming from $\mathrm{W}$ (+jets) or $\overline{\mathrm{t}}$ production, is efficiently removed by requiring that the transverse mass $m_{\mathrm{T}}$ is above the $\mathrm{W}$ mass. This variable is defined as $m_{\mathrm{T}} \equiv \sqrt{2 E_{\mathrm{T}}^{\mathrm{miss}} p_{\mathrm{T}}^{l}(1-\cos (\Delta \phi))}$, where $p_{\mathrm{T}}^{l}$ is the transverse momentum of the lepton and $\Delta \phi$ the difference in the azimuthal angle between the lepton and the $E_{\mathrm{T}}^{\text {miss }}$ direction. The $m_{\mathrm{T}}$ variable has an end-point for backgrounds containing a single leptonically decaying $\mathrm{W}$ boson, while signal events contain additional $E_{\mathrm{T}}^{\text {miss }}$ due to the LSPs, leading to an excess at large $m_{\mathrm{T}}$. When two leptons are considered the best discriminant variables are the generalized transverse masses $m_{\mathrm{T} 2}$ [36-38] and $m_{\mathrm{CT}}[39,40]$. It can be applied for example to search for direct slepton production $\tilde{1}^{+} \tilde{1}^{-} \rightarrow 1_{1} 1_{2} \tilde{\chi}_{1}^{0}\left(\mathrm{p}_{1}\right) \tilde{\chi}_{1}^{0}\left(\mathrm{p}_{2}\right) \cdot m_{\mathrm{T} 2}$ minimises the larger of the two transverse masses and is defined as

$m_{\mathrm{T} 2}^{2}=\min _{\mathbf{p}_{\mathbf{1} \mathrm{T}}+\mathbf{p}_{\mathbf{2}_{\mathrm{T}}}=\mathbf{E}_{\mathrm{T}}^{\mathrm{miss}}}\left\{\max \left(m_{\mathrm{T}}^{2}\left(1_{1}, \mathrm{p}_{1}\right), m_{\mathrm{T}}^{2}\left(1_{2}, \mathrm{p}_{2}\right)\right)\right\}$

while $m_{\mathrm{CT}}$ is a simple combination of the visible decay products and is defined as

$m_{\mathrm{CT}}^{2}=\left[E_{\mathrm{T}}\left(\mathrm{l}_{1}\right)+E_{\mathrm{T}}\left(\mathrm{l}_{2}\right)\right]^{2}-\left[\mathbf{p}_{\mathrm{T}}\left(\mathrm{l}_{1}\right)-\mathbf{p}_{\mathrm{T}}\left(\mathrm{l}_{2}\right)\right]^{2}$, 
with $E_{\mathrm{T}}=\sqrt{p_{\mathrm{T}}^{2}+m^{2}}$. In both cases-assuming that leptons are massless- a signal end-point is defined by $\left(m_{\widetilde{1}^{ \pm}}^{2}-\right.$ $\left.m_{\widetilde{\chi}_{1}^{0}}^{2}\right) / m_{\widetilde{1}^{ \pm}}$while the $\overline{t t}^{\prime}$ s end-point is $\left(m_{\mathrm{t}}^{2}-m_{\mathrm{W}}^{2}\right) / m_{\mathrm{t}} \sim$ $135 \mathrm{GeV}$, providing a very powerful discriminating variable for open spectra, i.e. $m_{\tilde{1}^{ \pm}}-M_{\widetilde{\chi}_{1}^{0}}>\mathrm{O}(100 \mathrm{GeV})$. This approach can be applied to all sparticle direct production (where the sparticle decays as SM partner and the LSP). As for $m_{\mathrm{eff}}$, if SUSY is discovered this will provide a way to measure sparticle masses. Other possibilities exist for mass constraining variables and are described in Ref. [41].

\subsection{Background modeling}

As just discussed, the signal regions are generally located in extreme regions of the phase space which are not necessarily well understood and described in simulation. As a consequence, the number of remaining background events in the signal region needs to be estimated as precisely as possible (to increase the sensitivity to the SUSY signal), preferably without relying only on simulation. Several methods using data in the background determination are possible, e.g. a semi data-driven approach using background enriched 'control' regions (CR) in data, or even a fully data-driven approach.

The fully data-driven approach is particularly suited for background processes with very large cross sections and fake $E_{\mathrm{T}}^{\text {miss }}$-like dijet production, fake lepton/photon in leptonic/photonic channels and long-lived particle searches. The first type of background is estimated with the jet smearing method, where the jet response for well-measured and badly measured jets is estimated in dedicated samples (see more details in [42]). The second type of background is estimated by counting the number of leptons/photons passing a loose lepton/photon selection but failing a tight one. With the measure of the true and fake lepton/photon efficiency to pass or fail the tight criteria from dedicated orthogonal samples, it is possible to estimate the number of fake leptons/photons in the tight selection. Finally, background to long-lived particles is estimated with a template method.

The semi data-driven approach is particular suited for SM processes with large cross section, as top-quark, W-, or Zboson production. The definition of the control region is a trade-off between kinematic requirements as similar as possible to the signal region to minimize systematics, the highest achievable purity and minimization of the contamination from potential signal. This is illustrated in Fig. 10. The simulation is normalized to the event yield in the control region (scale factor), and the background in the signal region is estimated by extrapolating the background level via simulation from the control region to the signal region (transfer factor).

Determination from Monte Carlo simulations only is generally adequate for backgrounds that are expected to be very small in the signal region (e.g. diboson production for strong

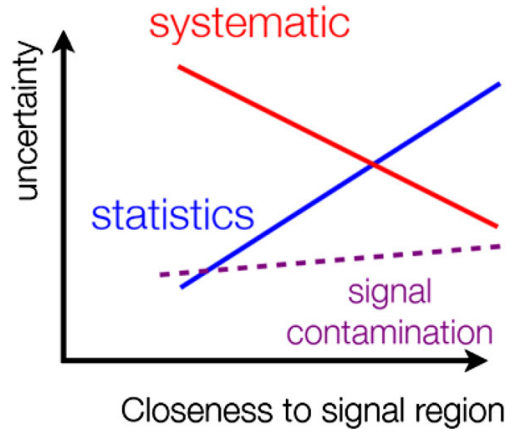

Fig. 10 Parameters entering in the design of a control region

SUSY searches), or for rare processes with very small cross sections (e.g. t t $\mathrm{t} H$ or triboson production).

Monte Carlo (MC) simulated event samples therefore play a central role in SUSY analyses. They allow not only to develop and validate the analysis procedure but also, in many cases, to evaluate the SM backgrounds, and to calculate the acceptance and efficiency of the studied signal samples. SUSY analyses rely heavily on the progress made in the last 20 years in the calculations and simulation of high $Q^{2}$ processes in hadronic collisions. Simulated samples of

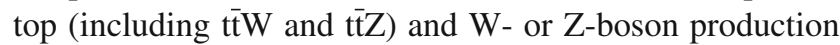
with multiple jets are produced with multi-parton generators such as ALPGEN [43], SHERPA [44] or MADGRAPH [45], with (in some cases) up to six additional partons in the matrix element. The next-to-leading-order (NLO) generators MC@NLO [46] and POWHEG [47-49] are generally used for top or diboson production. Parton shower and fragmentation processes are simulated with either HERWIG [50] or PYTHIA [51].

All SM backgrounds are then fed into a GEANT4-based [52] model of the CMS or ATLAS detector. Due to the large amount of signal points, the signal events are usually processed with a fast detector simulation [53,54]. The effect of multiple proton-proton collisions from the same or different bunch crossings is included in all simulations by overlaying minimum bias events onto hard-scattering events. The distribution of the number of interactions per bunch crossing is reweighted in the simulation to match the data.

Systematics are often estimated by comparing two generators of the same type, by using different parton distribution functions, and by changing the factorization, renormalization, and matching scales. The choice of the generator is made case-by-case and is analysis dependent: RPC strong production searches with a large number of jets generally use multi-parton generators, whereas EW production searches preferably use next-to-leading order generators.

\subsection{Limit extraction}

After all cuts have been applied, the number of data events $n$ is counted in the signal region ('cut-and-count' method). It is 
compared to the expected number of SM events to enter the signal region. For that, a likelihood function for observing $n_{\mathrm{B}}$ background events in the signal region is constructed as the product of Poisson probability distributions for event counts in the signal region and each of the main control regions, and of constraints for systematic uncertainties on the expected yields, called nuisance parameters. ${ }^{4}$ The Poisson probability density functions also include free parameters, for example to scale the expected contributions from the major backgrounds. The free parameters and nuisance parameters are adjusted to maximize the likelihood function. The result of the likelihood fit includes therefore a set of estimates and uncertainties for the background in the $\mathrm{SR}^{5}$. The significance of an excess is the probability that a background-only experiment is more signal-like than observed and is computed following the CLs prescription [55].

If no excess is observed, limits at $95 \%$ confidence level (CL) are set. Note that to obtain more constraining limits, some analyses make use of the discriminant variable shape. For each public result, efficiency maps are provided to allow theorists to test their own models. Following an ATLASCMS agreement, the numbers quoted for exclusion refer to the observed limit minus one standard deviation.

\subsection{SUSY models for interpretation}

The sensitivity of the SUSY searches are estimated by three complementary approaches, given that it is not possible to cover the entire parameter phase space.

First, constrained SUSY models, where boundary conditions at a high energy scale reduce the number of parameters to a few making it realistic to scan systematically, are tested. Benchmark models are MSUGRA/CMSSM [5660], minimal GMSB [61] and AMSB [62,63] models. Each model corresponds to a particular SUSY breaking messenger: gravity for the former and the latter and gauge bosons for GMSB. Similarly the LSP is generally the bino-like $\tilde{\chi}_{1}^{0}$ for MSUGRA/CMSSM, the wino-like $\tilde{\chi}_{1}^{0}$ for AMSB, and always the gravitino for GMSB.

Second, we have topological or simplified models [6466] where only a few SUSY particles are involved, while the masses of all other SUSY particles are set to multi$\mathrm{TeV}$ values, out of range at the LHC. The cascade decay of the remaining particles to the LSP, typically with zero or one intermediate step, is only characterized by the particle masses. These models are particularly suited for direct sparticle production.

\footnotetext{
${ }^{4}$ Nuisance parameters are modeled by a Gaussian probability density function with a width given by the size of the uncertainty.

5 The procedure is checked by building 'validation regions' in between the control and the signal and comparing with data.
}

Finally, results can also be interpreted in phenomenological MSSM (pMSSM) [67] models where the number of MSSM parameters is reduced to 19 by assuming the absence of new sources of flavor changing neutral currents and CP violation and universality of the first and second generation. By sampling a limited number of pMSSM parameters, the sensitivity of the searches to more 'realistic' configurations of SUSY particle masses and branching ratios can be assessed.

\section{Gluino and first/second generation of squarks}

In the MSSM, TeV-scale squarks and gluinos produced in pp collisions will decay promptly in long decay chains containing mainly quark and gluon jets and the LSP. SUSY events are therefore characterized by multiple energetic jets as well as transverse missing energy $\left(E_{\mathrm{T}}^{\text {miss }}\right.$ ) originating from the undetected LSP energies; see Fig. 5. Depending on the sparticle present (or not) in between the squarks/gluinos and the LSP, charged lepton(s) and/or photons could also appear in the cascade. This section summarizes the present status of searches for gluinos and first/second-generation squarks when the $\tilde{\chi}_{1}^{0}$ is the LSP (Sect. 4.1) and when the gravitino is the LSP (Sect. 4.2).

\subsection{SUSY models with $\tilde{\chi}_{1}^{0}$ as LSP}

To improve the sensitivity to these models, searches are usually divided in lepton-veto (Sect. 4.1.1) and leptonic (Sect. 4.1.2) searches. The former target more inclusive or generic scenarios, while the latter are generally more optimal for specific models. In both cases, requiring that some jets are originating from a $b$ quark can increase the sensitivity (Sect. 4.1.3). A summary is given in Sect. 4.1.4.

\subsubsection{Lepton-veto searches}

The preferred gluino (squark) decay modes are $\widetilde{\mathrm{g}} \rightarrow \mathrm{q} \widetilde{\mathrm{q}}$ $\left(\widetilde{\mathrm{q}} \rightarrow \mathrm{q} \widetilde{\chi}_{1}^{0}, \mathrm{q} \widetilde{\mathrm{g}}\right)$, which generate signatures with two to $\geq 10$ jets. Low jet multiplicities probe squark-squark (two jets or more), squark-gluino (three jets or more) or gluino-gluino (four jets or more) production. Additional jets compared to the tree level processes originate from initial- and final-state radiation jets (ISR/FSR) or from the presence of a top quark in the decay chain. In the last case, an increase of sensitivity is possible by requiring the presence of one or several b-tagged jets (see Sect. 4.1.3 for more details).

Several optimizations are possible, depending on the discriminating variables chosen, and this section only discusses the already published results based on rectangular cuts on $E_{\mathrm{T}}^{\text {miss }}$ and $H_{\mathrm{T}}$ [68], $H_{\mathrm{T}}^{\text {miss }}$ and $H_{\mathrm{T}}$ [69] on one side and 


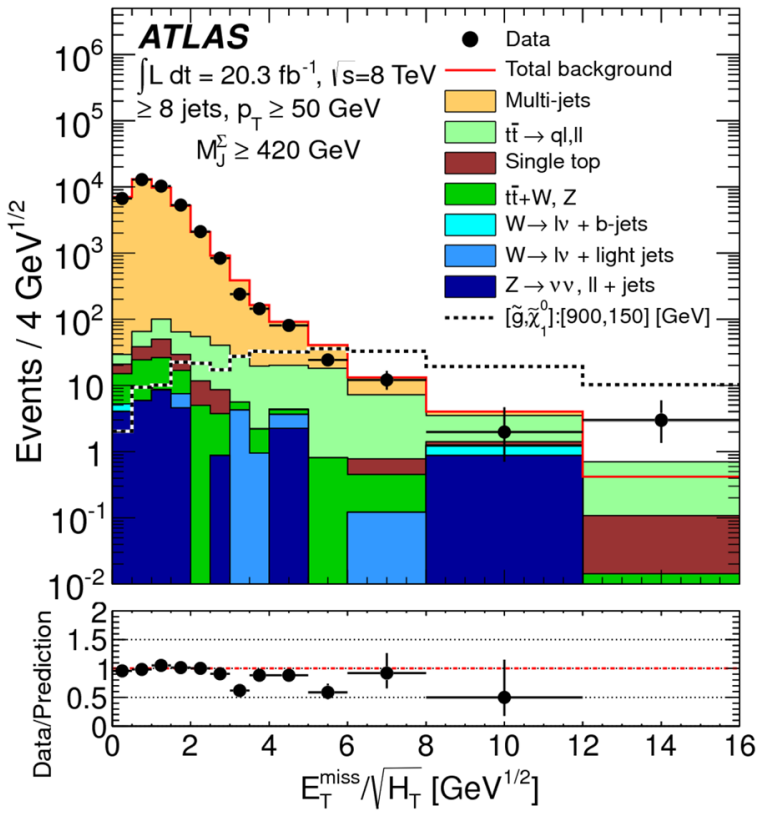

Fig. 11 Example of a discriminating variable: the $E_{\mathrm{T}}^{\text {miss }}$ significance from [70]

$E_{\mathrm{T}}^{\text {miss }}$ significance [70] on the other side. As an example Fig. 11 shows the $E_{\mathrm{T}}^{\text {miss }}$ significance distribution. The SM background, composed of $\mathrm{W}+$ jets, $\mathrm{Z}(\rightarrow v v)+$ jets, $\mathrm{t} \overline{\mathrm{t}}$ and QCD multi-jets, peaks at low $E_{\mathrm{T}}^{\text {miss }}$ significance value. A typical SUSY signal, where gluinos of mass $900 \mathrm{GeV}$ are pair produced and decay each to a tt pair and a LSP of mass of $150 \mathrm{GeV}$, leads to much higher values. The signal region is defined as $E_{\mathrm{T}}^{\text {miss }} / \sqrt{H_{\mathrm{T}}}>4 \mathrm{GeV}^{1 / 2}$.

In all lepton-veto analyses, the challenge is to properly estimate backgrounds that are poorly modeled by Monte Carlo simulations: the QCD multi-jet background is obtained with a jet smearing method or a template method for the $E_{\mathrm{T}}^{\text {miss }}$ significance search. $\mathrm{Z}(\rightarrow v v)+$ jets estimate relies on a close-by Standard Model process like $\gamma+$ jets or $\mathrm{Z}(\rightarrow \mu \mu)+$ jets samples. $\mathrm{W}+$ jets and $\mathrm{t} \overline{\mathrm{t}}$ are estimated by designing control regions close to the signal regionsrequiring one lepton for example. Note that other searches with $m_{\text {eff }}$ or Razor as discriminating variables will become available soon. Searches with $\alpha_{\mathrm{T}}$ are only available for half the luminosity of the 2012 data [71]. All these analyses are generally more powerful than [68-70] but will not change the overall picture given in the rest of this section.

All these searches are particularly efficient for open spectra where the mass difference between the LSP and the gluino/squark is large $(\Delta M>\mathrm{O}(500) \mathrm{GeV})$, providing highenergetic jets. This is for example the case in the constrained SUSY model MSUGRA/CMSSM where the two most relevant parameters, the universal scalar and fermion masses at Grand Unified Theory (GUT) scale, $m_{0}$ and $m_{1 / 2}$, are var-

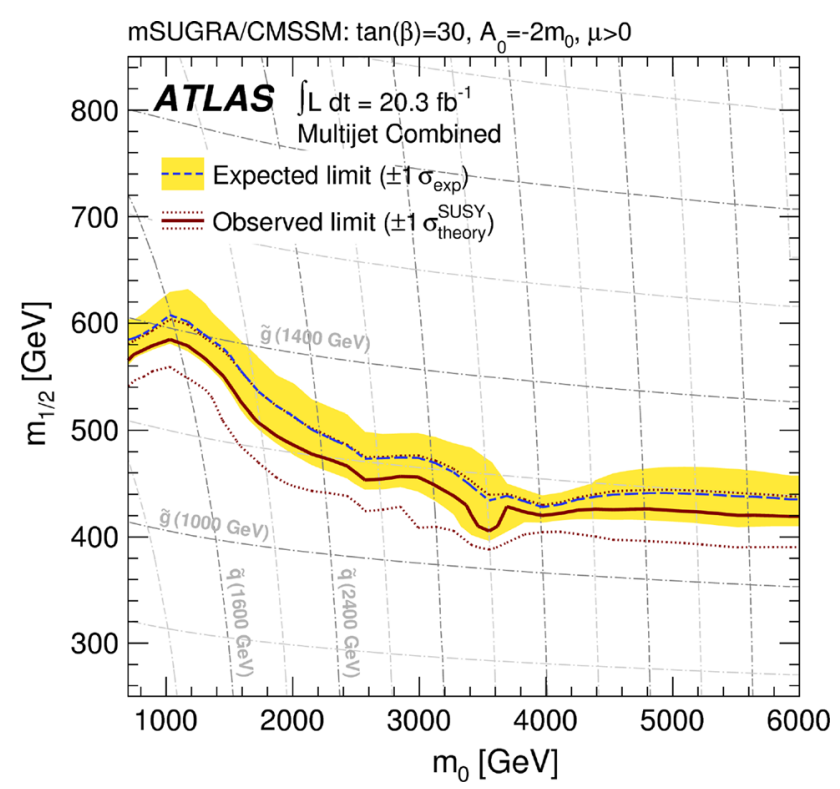

Fig. 12 Typical exclusion limit at 95\% CL from the lepton-veto inclusive search in the MSUGRA/CMSSM scenario [70]

ied to construct a grid of points. ${ }^{6}$ Figure 12 shows the limits obtained with the $E_{\mathrm{T}}^{\text {miss }}$ significance search. Squark and gluino masses at the EW scale are proportional to the $m_{0}$ and $m_{1 / 2}$ parameters and shown by the isolines, which indicate that gluino and degenerate squark masses below $1.2 \mathrm{TeV}$ are excluded. For equal degenerate squark and gluino masses a limit of $1.3 \mathrm{TeV}$ is reached.

Another way to represent the power of the lepton-veto searches is to use simplified models where $\widetilde{\mathrm{g}} \rightarrow \mathrm{q} \overline{\mathrm{q}} \widetilde{\chi}_{1}^{0}$ decays are enforced. Here again a limit of $1.2 \mathrm{TeV}$ on the gluino mass is obtained (for massless LSPs); see Fig. 13. However, for more compressed spectra the limits degrade and LSP masses cannot be excluded beyond $500 \mathrm{GeV}$. A similar situation occurs when considering mass-degenerate light flavor squarks forced to decay as $\widetilde{\mathrm{q}} \rightarrow \mathrm{q} \widetilde{\chi}_{1}^{0}$; see Fig. 14 . Compared to the gluino situation, the limits are degraded to $800 \mathrm{GeV}$ for squark masses (again in the case of massless LSPs), and LSP masses cannot be excluded beyond $300 \mathrm{GeV}$. These limits are reduced to 400 and $100 \mathrm{GeV}$, respectively, when only one light squark is considered.

Overall these results represent an increase of about one order of magnitude compared to the previous limits from Tevatron and LEP. From the lepton-veto analyses, the strongest limits are obtained for the gluino in open SUSY spectra, and they exclude a large part of the favored region from naturalness (cf. Fig. 3).

\footnotetext{
6 The other fixed parameters $\left(\tan \beta, A_{0}\right.$, and the sign of $\mu$ ) are chosen to accommodate a $126 \mathrm{GeV}$ Higgs mass.
} 


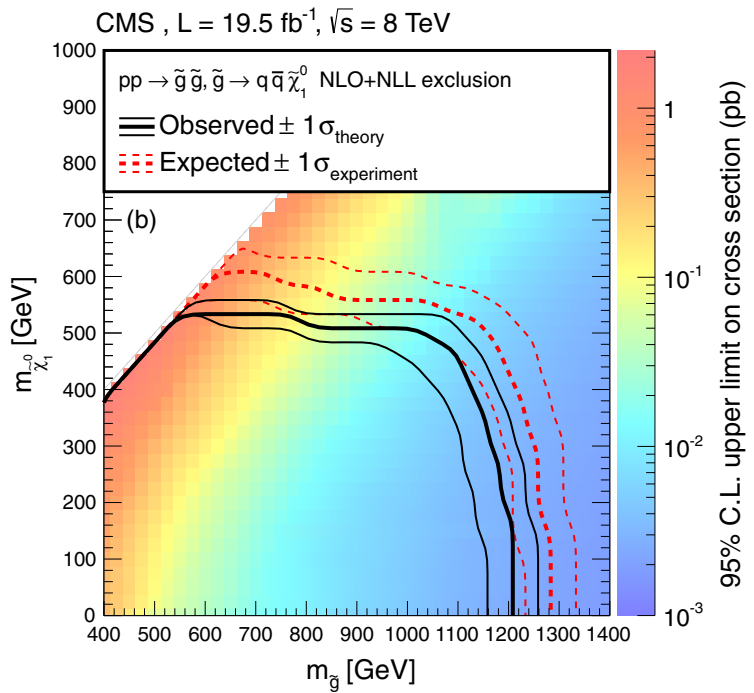

Fig. 13 Exclusion limits at $95 \% \mathrm{CL}$ on the gluino-gluino production in the gluino-LSP mass plane [68]. The gluino always decays as $\widetilde{\mathrm{g}} \rightarrow$ $\mathrm{q} \overline{\mathrm{q}} \widetilde{\chi}_{1}^{0}$ and all other SUSY particles are decoupled

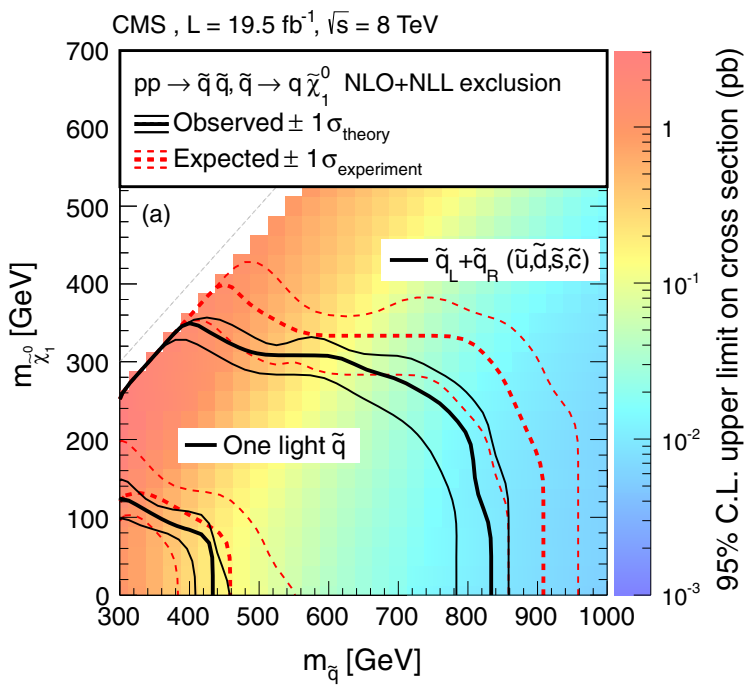

Fig. 14 Exclusion limits at $95 \% \mathrm{CL}$ on the squark-squark production in the squark-LSP mass plane [68]. The squark always decays as $\widetilde{\mathrm{q}} \rightarrow$ $\mathrm{q} \widetilde{\chi}_{1}^{0}$ and all other SUSY particles are decoupled

\subsubsection{Leptonic searches}

Requiring one or two isolated leptons (electron or $\mu$ ) on top of multi-jets and $E_{\mathrm{T}}^{\text {miss }}$ allows to probe other regions of parameter space, and especially more compressed mass spectra. The lepton generally comes from $\mathrm{W}$ leptonic decays originating from chargino, top or from slepton decay. Experimental challenges drastically change: lepton triggers can be exploited and requirements on jet kinematics can be reduced. Lowering cuts on $E_{\mathrm{T}}^{\mathrm{miss}}$ and $H_{\mathrm{T}}$ is possible since the multi-jet QCD background is naturally suppressed by the presence of iso- lated lepton(s). Very soft leptons (in the 6-25 GeV range) are also considered to probe the compressed gluino-charginoLSP case [72]. Finally, other variables exist like the transverse mass $m_{\mathrm{T}}$, which efficiently reduces $\mathrm{t} \overline{\mathrm{t}}$ and $\mathrm{W}+$ jets backgrounds by requiring $m_{\mathrm{T}}>m_{\mathrm{W}}$, as discussed in Sect. 3.2. This allows one to compensate the loss due to the leptonic branching ratio(s) when comparing with lepton-veto analyses. While this works well for single-lepton analyses to be competitive with lepton-veto analyses, this is generally not sufficient when two leptons are considered. ${ }^{7}$ In that case, it is more advantageous to consider two leptons of same-sign since this signature is almost not produced by SM processes and appears naturally in many SUSY decays. The two reasons are: (i) gluinos are Majorana particles and produced in pairs, therefore if leptons are present in each leg they have $50 \%$ probability to be of same-sign, (ii) multi-W final states occur frequently through top and chargino decays and leptonic $\mathrm{W}$ decay will ensure the presence of two same-sign leptons in most cases. For a same-sign dilepton analysis, the main background is caused by the $\operatorname{rare} \operatorname{tt} X\left(X=\mathrm{h}^{0}, \mathrm{Z}, \mathrm{W}\right)$ SM processes, fake leptons and mis-measured lepton charge because of the process $1 \rightarrow 1 \gamma \rightarrow 11 \bar{l}$ where $\overline{1}$ inherits most of the energy of the original lepton.

\subsubsection{Multi b-tagged jet searches}

As for leptons, identifying b-tagged jets in the multi-jet final states can be a precious help, especially together with leptons. This is particularly true for the decay $\widetilde{\mathrm{g}} \rightarrow \widetilde{\mathrm{t}} \rightarrow \mathrm{t} \widetilde{\chi}_{1}^{0}$, favored by the natural mass spectrum. This will provide four tops $+E_{\mathrm{T}}^{\text {miss }}$ final states. Several dedicated analyses have been designed to reach this striking final state and obtain extra sensitivity compared to lepton-veto analyses described in Sect. 4.1. Reducing the dominant $\mathrm{t} \overline{\mathrm{t}} \rightarrow \mathrm{W}^{+} \mathrm{W}^{-} \mathrm{b} \overline{\mathrm{b}}$ background is possible when considering (i) a single isolated lepton and at least five jets, two or three of which are identified as b-tagged jets [73], (ii) two same-sign leptons with one, two or three b-tagged jets [74]. ${ }^{8}$ The best sensitivity is obtained by the former, which can exclude gluino masses up to $1.3 \mathrm{TeV}$ for LSP masses below $600 \mathrm{GeV}$, assuming a $100 \%$ branching ratio for the decay $\widetilde{\mathrm{g}} \rightarrow \widetilde{\mathrm{tt}} \rightarrow \mathrm{tt} \widetilde{\chi}_{1}^{0} \widetilde{\chi}_{1}^{0}$. The same-sign dilepton analysis allows one to probe the compressed spectra part when one top is off-shell. The relative strengths of the different analyses for this model can be judged from Fig. 15 . Similar results are obtained for $\widetilde{\mathrm{g}} \rightarrow \mathrm{b} \widetilde{b}_{\mathrm{L}} \rightarrow \mathrm{b} \overline{\mathrm{b}} \widetilde{\chi}_{1}^{0} \widetilde{\chi}_{1}^{0}$.

\footnotetext{
7 This final state could be very useful to determine SUSY parameters but is generally not for discovery.

8 The case of three b-tagged jets without a lepton is also considered but no public results exist yet at $\sqrt{s}=8 \mathrm{TeV}$.
} 


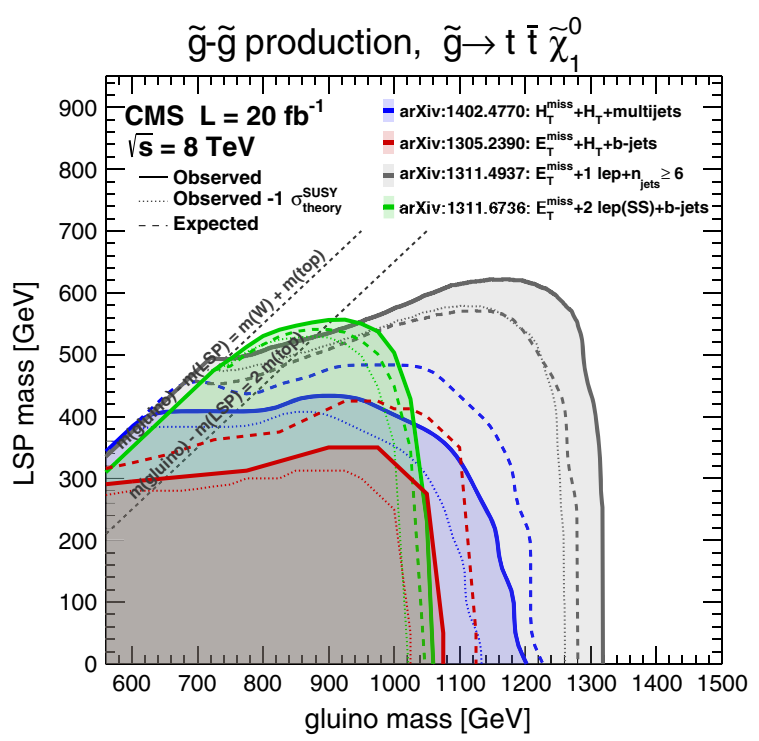

Fig. 15 Exclusion limits at $95 \% \mathrm{CL}$ on the gluino-gluino production in the gluino-LSP mass plane for a simplified model where gluino decays promptly via off-shell top squark and a LSP. The other sparticle masses are assumed to be decoupled. Four different analyses are shown

\subsubsection{Summary}

Ultimate limits from the first LHC run on gluino and squark masses when the $\widetilde{\chi}_{1}^{0}$ is the LSP will be obtained by combining all lepton-veto and leptonic analyses-many results are still to come. However, it is already fair to say that the most important constraint from the LHC experiments is the exclusion of the gluino mass below $1 \mathrm{TeV}$ for open spectra. This fairly generic limit excludes a great part of the favored region from naturalness (cf. Fig. 3). Since the gluino mass is governed by only one parameter $\left(M_{3}\right)$ and enters in the top squark and EWKino masses through loop corrections, this has the general effect to pull up the whole natural spectrum [75]. Compressed spectra are still poorly explored, but more work is still going on to exploit monojet-like final states provided by ISR/FSR. Extra sensitivity will also be brought by analyses based on reduced lepton and jet thresholds - this is possible when the peak luminosity decreases at the end of a LHC run. Another important information is coming from the LSP mass constraint which can reach up to $600 \mathrm{GeV}$ in leptonic analyses, though it is less generic than the gluino limit. Finally, squark-mass constraints are more model-dependent and single squark-mass limits are at most of the order of $500 \mathrm{GeV}$.

\subsection{SUSY models with $\widetilde{\mathrm{G}}$ as LSP}

SUSY scenarios where the gravitino is the LSP generate a variety of final states driven by the NLSP-gravitino coupling.
While some of these final states are common with Sect. 4.1, when the NLSP is the gluino or a squark, some need the development of new dedicated analyses, when NLSP is a slepton, chargino or neutralino. The most natural solution is $\tilde{\chi}_{1}^{0}$ as the NLSP, leading to final states with extra $\gamma, \mathrm{Z}$ or $\mathrm{h}^{0}$, depending on the SUSY parameters.

Among all possible final states, the ones containing photons, jets and $E_{\mathrm{T}}^{\text {miss }}$ from the gluino/squark cascade could have escaped searches described in Sect. 4.1. In this case, the most dangerous background is caused by multi-jet or $\gamma+$ jets events when the jet is mimicking a photon. This background type can be drastically reduced with a good photon/jet rejection-measured to be $O\left(10^{4}\right)$ in the $H \rightarrow \gamma \gamma$ channel—and by requiring a high value for $E_{\mathrm{T}}^{\text {miss }}$. The other type of background are SM electroweak processes, especially $\mathrm{W}\left(\rightarrow \mathrm{e} v_{\mathrm{e}}\right) \gamma$, where the electron is reconstructed as a photon and true $E_{\mathrm{T}}^{\text {miss }}$ is caused by the neutrino. In both cases, datadriven methods are used for the background estimate. As an example, the distribution of $E_{\mathrm{T}}^{\text {miss }}$, the main discriminating variable for the $\gamma \gamma$ searches is shown in Fig. 16. A high sensitivity is observed for the SUSY signal at high $E_{\mathrm{T}}^{\text {miss }}$. Assuming a bino-like $\widetilde{\chi}_{1}^{0}$ NLSP, Fig. 17 shows that it is possible to exclude gluino masses below $1 \mathrm{TeV}$ regardless of the NLSP mass [76].

When the $\widetilde{\chi}_{1}^{0}$ is higgsino-like, the preferred solution from naturalness arguments, $\widetilde{\chi}_{1}^{0} \rightarrow \mathrm{h}^{0}(\rightarrow \mathrm{b} \overline{\mathrm{b}}) \widetilde{\mathrm{G}}$ final states is expected. No analysis presently attempts to search for a $4 \mathrm{~b}+$ $E_{\mathrm{T}}^{\text {miss }}$ final state. However, assuming that $\tilde{\chi}_{1}^{0}$ is also partly bino-like, the signature will be $\gamma+\mathrm{b}+E_{\mathrm{T}}^{\text {miss }}$ and this has been searched for. A second photon and a lepton veto are applied to remain orthogonal with other searches and remove final states with leptonic W decays [78]. In this case also the gluino mass limit reaches values around $1 \mathrm{TeV}$, as shown in Fig. 18.

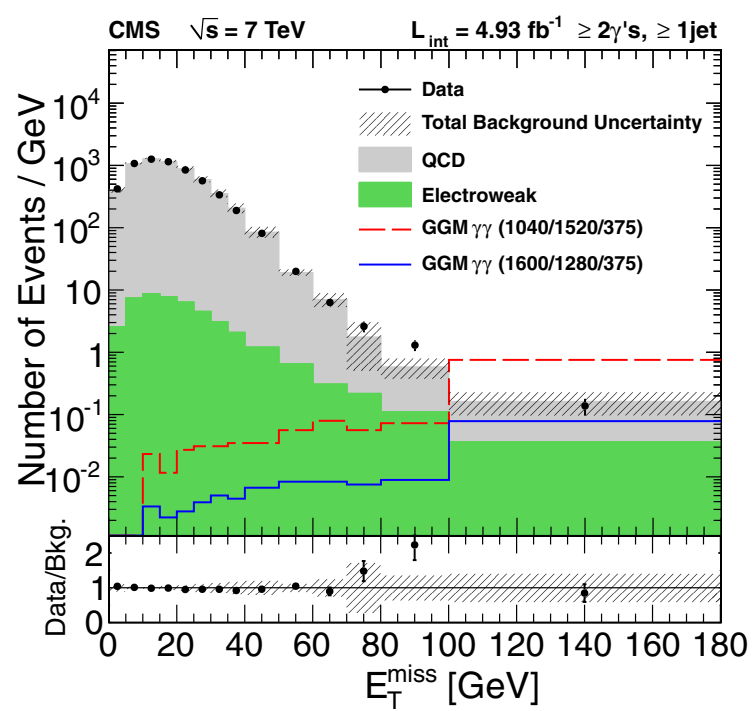

Fig. $16 E_{\mathrm{T}}^{\mathrm{miss}}$ distribution in the diphoton analysis [77] 


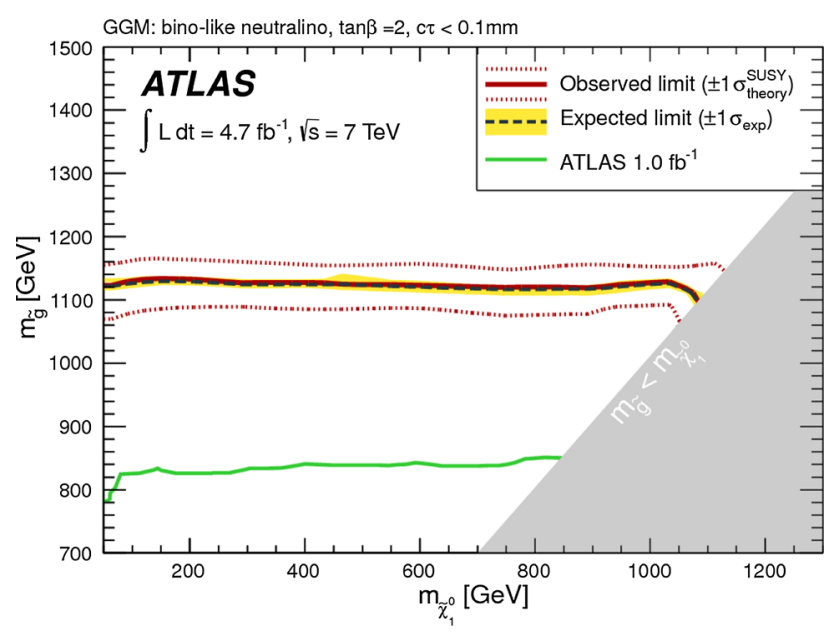

Fig. 17 Exclusion limits at 95\% CL on the gluino-gluino production in the gluino-LSP mass plane from the diphoton $+E_{\mathrm{T}}^{\text {miss }}$ analysis [76]. The simplified model assumes a bino-like NLSP and a gravitino LSP. The other sparticle masses are assumed to be decoupled

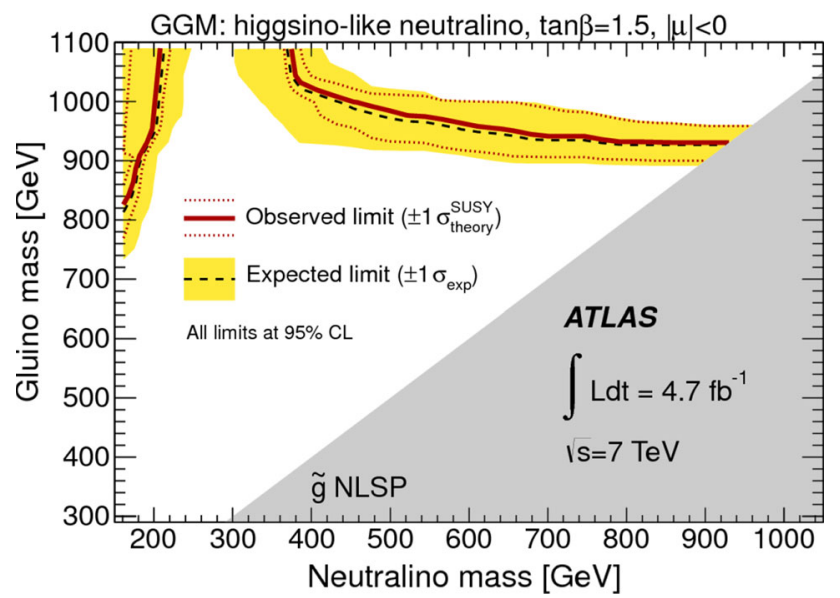

Fig. 18 Exclusion limits at 95\% CL on the gluino-gluino production in the gluino-LSP mass plane from the $\gamma+\mathrm{b}+E_{\mathrm{T}}^{\mathrm{miss}}$ analysis [78]. The simplified model assumes a higgsino-like NLSP. The other sparticle masses are assumed to be decoupled

All other NLSP cases are generally already well covered by the searches discussed in Sect. 4.1 [12]. However, it is possible to gain a bit in sensitivity by searching for one or two $\tau+$ jets $+E_{\mathrm{T}}^{\text {miss }}$ final states $[79,80]$, especially when $\tilde{\tau}$ is the NLSP or co-NLSP with other sleptons. In this case, it is possible to combine with a 2-lepton + jets $+E_{\mathrm{T}}^{\text {miss }}$ search [72] and even a 3-lepton + jets $+E_{\mathrm{T}}^{\text {miss }}$ search [81]. In all cases, the gluino mass limit is always above $1 \mathrm{TeV}$.

As for models with the $\widetilde{\chi}_{1}^{0}$ as LSP, SUSY scenarios where the $\widetilde{\mathrm{G}}$ is the LSP provide strong constraints on sparticle masses and particularly on the gluino mass which is generally excluded below $1 \mathrm{TeV}$ whatever the NLSP nature and mass are. Less stringent limits are obtained for squarks. It is worth to note that these conclusions are based on $7 \mathrm{TeV}$ results-presently only one $8 \mathrm{TeV}$ result is available.

\section{Third generation of squarks}

As already discussed in Sect. 2, naturalness predicts light third-generations squarks. Another motivation for the thirdgeneration squarks to be the lightest colored sparticles is that the squark mass eigenstates $\left(\widetilde{\mathrm{q}}_{1}, \widetilde{\mathrm{q}}_{2}\right)$ depend on orthogonal combinations of the gauge eigenstates $\left(\widetilde{\mathrm{q}}_{R}, \widetilde{\mathrm{q}}_{L}\right)$, e.g. for the lighter squark given by $\widetilde{\mathrm{q}}_{1}=\widetilde{\mathrm{q}}_{\mathrm{L}} \cos \left(\Theta_{\widetilde{\mathrm{q}}}\right)+\widetilde{\mathrm{q}}_{\mathrm{R}} \sin \left(\Theta_{\widetilde{\mathrm{q}}}\right)$. The off-diagonal elements of the mass matrix $\Theta_{\widetilde{q}}$ are proportional to the mass of the SM partner particle, the Higgs-related parameters $\mu$ and $\tan \beta$. Therefore, the mass of the $\widetilde{t}_{1}$, predominantly $\widetilde{t}_{R}$, can be small due to the large top quark mass, and the $\widetilde{\mathrm{b}}_{1}$ mass is expected to be light for large $\tan \beta$. For small $\tan \beta$ the $\widetilde{b}_{\mathrm{L}}$ is still expected to be small due to the effects of the large top Yukawa coupling, as it is part of the doublet containing $\widetilde{t}_{\mathrm{L}}$.

The following two sections review the status of the searches for models with $\widetilde{\chi}_{1}^{0}$ being the LSP (Sect. 5.1), and the $\widetilde{\mathrm{G}}$ being the LSP (Sect. 5.2).

\subsection{SUSY models with $\tilde{\chi}_{1}^{0}$ as LSP}

If the third-generation squarks are lighter than gluinos, they are likely to appear in gluino decay chains. But if the gluino masses are too heavy to be produced at the LHC energy, searches for direct third-generation squark pair production might be the only way to observe them, even though these branching ratios are more than one order of magnitude lower than those for gluino-gluino production (see Fig. 4).

For top squarks, the possible decays and therewith connected search strategies depend on the masses of the accessible particles. Assuming that the $\tilde{\mathfrak{t}}_{1}$, the $\tilde{\chi}_{1}^{ \pm}$and the $\tilde{\chi}_{1}^{0}$ are the only accessible SUSY particles, the possible decays in the $m_{\tilde{\mathrm{t}}_{1}}-m_{\widetilde{\chi}_{1}^{0}}$ parameter plane are displayed in Fig. 19.

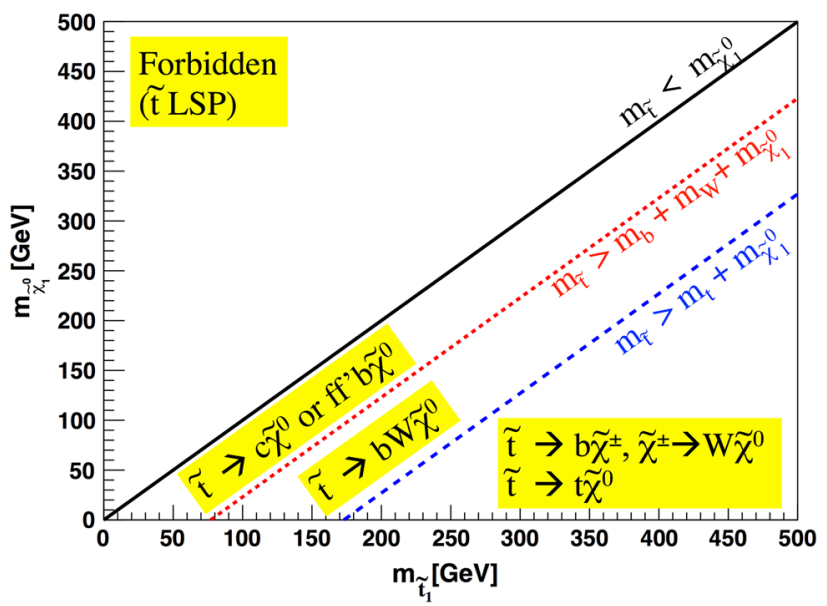

Fig. 19 Overview of the possible top squark decays depending on the mass of the $\widetilde{t}_{1}$ and the $\widetilde{\chi}_{1}^{0}$ 
The most obvious decay chain is given for $m_{\tilde{\mathrm{t}}_{1}}>m_{\mathrm{t}}+$ $m_{\widetilde{\chi}_{1}^{0}}$ : here the top squark will decay either like $\tilde{\mathrm{t}} \rightarrow \mathrm{t} \widetilde{\chi}_{1}^{0}$ or $\widetilde{\mathrm{t}} \rightarrow$ $\mathrm{b} \widetilde{\chi}_{1}^{ \pm}$, where $\tilde{\chi}_{1}^{ \pm} \rightarrow \mathrm{W}^{ \pm} \widetilde{\chi}_{1}^{0}$, with cross sections depending on the $\tilde{\chi}_{1}^{ \pm}$mass. In both cases this gives two $\mathrm{W}$ bosons, resulting in a large probability to have (at least) one electron or muon in the final state. Hence, the searches requiring zero or one lepton in the final state are the strongest. Zero-lepton searches are expected to have a slightly higher reach in the $\tilde{\mathrm{t}}_{1}$ mass for low $\tilde{\chi}_{1}^{0}$ masses, while one-lepton analyses tend to reach closer to the diagonal line at $m_{\widetilde{t}_{1}}-m_{\widetilde{\chi}_{1}^{0}}=m_{\mathrm{t}}$. The region around this line is very difficult to cover with current searches, as the kinematics of the decay are similar to the SM top decay kinematics. An option to test this parameter space exists for cases where the $\tilde{t}_{2}$ is not too heavy either. A decay chain to search for would be $\widetilde{\mathrm{t}}_{2} \rightarrow \widetilde{\mathrm{Zt}}_{1}$. Requiring same-flavor dileptons from the $\mathrm{Z}$ boson decay in addition to a lepton from the $\tilde{\mathrm{t}}_{1} \rightarrow \mathrm{t} \widetilde{\chi}_{1}^{0}$ decay provide a powerful background rejection [82].

When the $\widetilde{t}_{1}$ is lighter than the top, in the region defined by $m_{\mathrm{b}}+m_{\mathrm{W}}+m_{\widetilde{\chi}_{1}^{0}}<m_{\widetilde{t}_{1}}<m_{\mathrm{t}}+m_{\widetilde{\chi}_{1}^{0}}$ the top squark will decay as $\tilde{\mathrm{t}} \rightarrow \mathrm{b} \tilde{\chi}_{1}^{ \pm}$, with the subsequent decay $\tilde{\chi}_{1}^{ \pm} \rightarrow \mathrm{W}^{ \pm} \tilde{\chi}_{1}^{0}$. This can best be tested with an analysis requiring two leptons and two b-tagged jets, which also has sensitivity to the three-body decay $\widetilde{\mathrm{t}} \rightarrow \mathrm{bW}^{ \pm} \widetilde{\chi}_{1}^{0}$, which becomes important for high $\widetilde{\chi}_{1}^{ \pm}$ masses. Also a one-lepton search has sensitivity in this area, as shown below.

For $m_{\tilde{t}_{1}}<m_{\mathrm{b}}+m_{\mathrm{W}}+m_{\widetilde{\chi}_{1}^{0}}$, the $\widetilde{\mathrm{t}}_{1}$ is expected to decay to $c \widetilde{\chi}_{1}^{0}$. This case is best tested with a monojet analysis, which can contain a charm-tag as well, which is not yet published. The results of top squark searches are discussed in Sect. 5.1.1.

Searches for the decay of the bottom squark via $\widetilde{b}_{1} \rightarrow$ $\mathrm{b} \widetilde{\chi}_{1}^{0}$, are usually performed in zero-lepton analyses requiring two b-tagged jets, as no prompt leptons are expected from $\mathrm{b}$ decays. If $m_{\tilde{\mathrm{b}}_{1}}>m_{\mathrm{t}}+m_{\widetilde{\chi}_{1}^{0}}$, the decay $\widetilde{\mathrm{b}}_{1} \rightarrow \mathrm{t} \tilde{\chi}_{1}^{-}$, with $\tilde{\chi}_{1}^{-} \rightarrow \mathrm{W}^{-} \tilde{\chi}_{1}^{0}$, is open as well, and searches with lepton signatures are again advisable, e.g. a same-sign dilepton search is well suited due to the low SM background in this channel. For $\widetilde{b}$ decays to a bottom quark and $\widetilde{\chi}_{2}^{0}$, the $\widetilde{\chi}_{2}^{0}$ can decay with a certain probability to a $\mathrm{Z}$ or Higgs boson and $\widetilde{\chi}_{1}^{0}$. The additional boson could be tagged to further reduce the background. The results of bottom squark searches are discussed in Sect. 5.1.2.

\subsubsection{Search for direct top squark production}

The first search [83] discussed here is focused on the direct production of two top squarks, with two possible decay modes of the top squark: $\widetilde{\mathrm{t}} \rightarrow \mathrm{t} \widetilde{\chi}_{1}^{0}$ and $\widetilde{\mathrm{t}} \rightarrow \mathrm{b} \widetilde{\chi}_{1}^{ \pm}$, with $\tilde{\chi}_{1}^{ \pm} \rightarrow \mathrm{W}^{ \pm} \tilde{\chi}_{1}^{0}$, for which a one-lepton final state is a favorable final state.

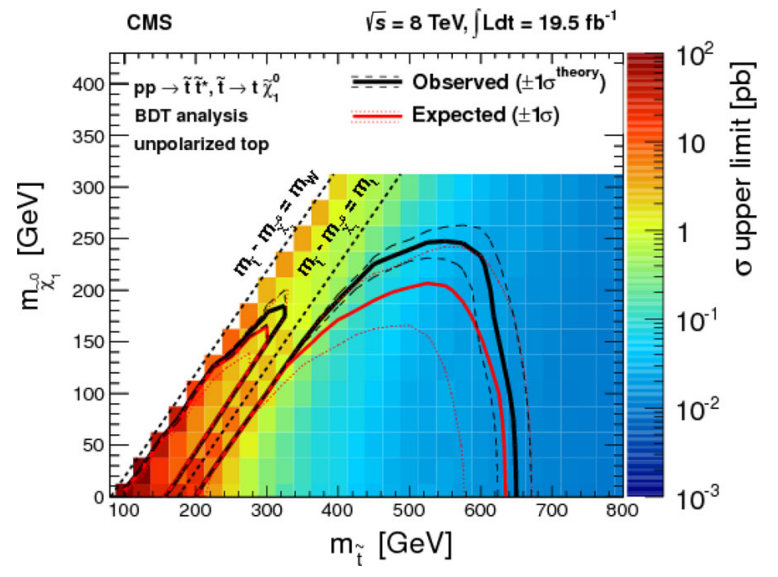

Fig. 20 Exclusion limits at $95 \% \mathrm{CL}$ on the top squark pair production in the top squark-LSP mass plane for a simplified model assuming $\widetilde{\mathrm{t}} \rightarrow \mathrm{t} \tilde{\chi}_{1}^{0}[83]$. All other sparticles are decoupled

The dominant tt $\mathrm{t}$ background, where both $\mathrm{W}$ bosons decay leptonically, is reduced by the quantity $m_{\mathrm{T} 2}^{\mathrm{W}}$, which is defined as the minimum mother particle mass compatible with all transverse momenta and mass-shell constraints [84]. This variable is similar to the $m_{\mathrm{T} 2}$ variable (cf. Sect. 3.2). $m_{\mathrm{T} 2}^{\mathrm{W}}$ has by construction an end-point at the top-quark mass in the case of a dileptonic top-quark decay, where one lepton is not identified or lies outside the acceptance of the analysis. In the search for the decay $\widetilde{t} \rightarrow \mathrm{t} \widetilde{\chi}_{1}^{0}$, the dilepton $\mathrm{t} \bar{t}$ background is further suppressed by requiring that three of the jets in the event are consistent with the $\mathrm{t} \rightarrow \mathrm{bW} \rightarrow \mathrm{bq} \overline{\mathrm{q}}$ decay chain.

The exclusion limits are shown in Fig. 20 for the simplified model describing $\widetilde{\mathrm{t}} \rightarrow \mathrm{t} \widetilde{\chi}_{1}^{0}$. Here the top is unpolarized, and a maximum limit of $620 \mathrm{GeV}$ for the top squark mass and of $225 \mathrm{GeV}$ for the $\tilde{\chi}_{1}^{0}$ mass can be set. In the case of $100 \%$ right-handed tops one would expect leptons with larger $p_{\mathrm{T}}$ leading to a larger acceptance, and hence to an extension of the limit at high masses by $25-50 \mathrm{GeV}$. Accordingly, a $100 \%$ left-handed top would reduce the limit by the same amount. Also, one has to take into account that the simplified model assumes a probably too optimistic branching fraction of $100 \%$. If the branching fraction would be reduced to $60 \%$ with no possibility to detect other decay chains, the excluded limit would drop to $m_{\mathfrak{t}}<500 \mathrm{GeV}$ and $m_{\widetilde{\chi}_{1}^{0}}<125 \mathrm{GeV}$.

Effects of similar size are also observed for the simplified model describing $\widetilde{\mathrm{t}} \rightarrow \mathrm{b} \widetilde{\chi}_{1}^{ \pm}$, with $\widetilde{\chi}_{1}^{ \pm} \rightarrow \mathrm{W}^{ \pm} \widetilde{\chi}_{1}^{0}$. Figure 21 shows this model for the unpolarized chargino, left-right symmetric $\mathrm{W} \tilde{\chi}_{1}^{0} \widetilde{\chi}_{1}^{ \pm}$coupling, and the mass parameter of the $\tilde{\chi}_{1}^{ \pm}$set to $m_{\widetilde{\chi}_{1}^{ \pm}}=x m_{\mathfrak{t}}+(1-x) m_{\widetilde{\chi}_{1}^{0}}$ with $x=0.5$. For a larger mass parameter (with $x=0.75$ ) the excluded top squark and $\widetilde{\chi}_{1}^{0}$ mass is shifted up by about $25-50 \mathrm{GeV}$, while for a lower mass parameter $(x=0.25)$ the limit becomes slightly weaker. For right-handed charginos and right-handed $\mathrm{W} \widetilde{\chi}_{1}^{0} \widetilde{\chi}_{1}^{ \pm}$couplings the limit is up to $50 \mathrm{GeV}$ stronger, while it is weaker for other combinations of polarizations of chargino 


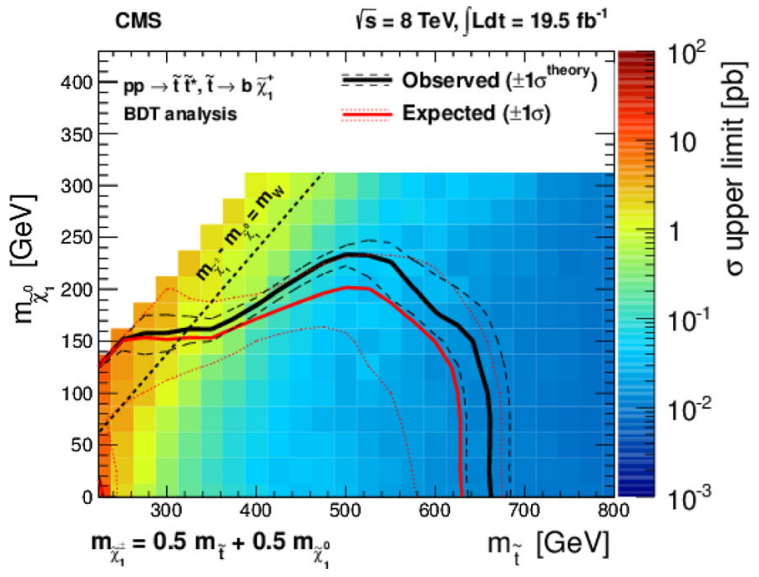

Fig. 21 Exclusion limits at $95 \% \mathrm{CL}$ on the top squark pair production in the top squark-LSP mass plane for a simplified model assuming $\widetilde{\mathrm{t}} \rightarrow \mathrm{b} \tilde{\chi}_{1}^{ \pm}$, with $\tilde{\chi}_{1}^{ \pm} \rightarrow \mathrm{W}^{ \pm} \tilde{\chi}_{1}^{0}$. The $\tilde{\chi}_{1}^{ \pm}$mass parameter is $x=0.5$ (see text) [83]. All other sparticles are decoupled

and $\mathrm{W} \widetilde{\chi}_{1}^{0} \widetilde{\chi}_{1}^{ \pm}$couplings. Searches for top squarks can also be performed in 2-lepton final states [85], and lead to slightly less stringent results, which are compatible with the 1-lepton search results.

In summary, top squarks around $600 \mathrm{GeV}$ can be excluded for $\widetilde{\chi}_{1}^{0}$ masses lower than $200 \mathrm{GeV}$, assuming that $\widetilde{\chi}_{1}^{ \pm}$and $\widetilde{\chi}_{1}^{0}$ are the only lighter SUSY particles, in which case the branching fractions for the investigated decays are large. This result excludes for the first time a large part of the phase space allowed by naturalness. Here it should be mentioned that the limit is not so strong for models with rich EWKino and slepton spectra with masses below the top squark mass. In such models many more decay chains may open up, leading partly to very soft objects in the final state and therefore deteriorating the acceptance and hence the achievable limit.

\subsubsection{Search for direct bottom squark production}

Assuming $\widetilde{b}_{1} \rightarrow \mathrm{b} \tilde{\chi}_{1}^{0}$, direct bottom squark production are searched for by requiring a lepton-veto, two b-tagged jets and a moderate amount of missing energy due to the LSPs $\left(E_{\mathrm{T}}^{\text {miss }}>150 \mathrm{GeV}\right)$ [86]. Further discriminating variables are the minimum angle $\Delta \phi$ between the $E_{\mathrm{T}}^{\text {miss }}$ vector and either of the three highest- $p_{\mathrm{T}}$ jets, which is expected to be larger for signal than for background from multi-jet events, and a requirement on the contransverse mass $m_{\mathrm{CT}}$ (cf. Sect. 3.2), which is displayed in Fig. 22.

Figure 23 shows the exclusion limit for direct bottom squark pair production. Bottom squark masses up to $620 \mathrm{GeV}$ and $\widetilde{\chi}_{1}^{0}$ masses up to $260 \mathrm{GeV}$ are excluded at $95 \% \mathrm{CL}$. Up to bottom squark masses of $300 \mathrm{GeV}$, mass differences of at least $50 \mathrm{GeV}$ between $\widetilde{b}$ and $\widetilde{\chi}_{1}^{0}$ can be excluded. Again, these limits correspond to a branching fraction of $100 \%$. If a lower

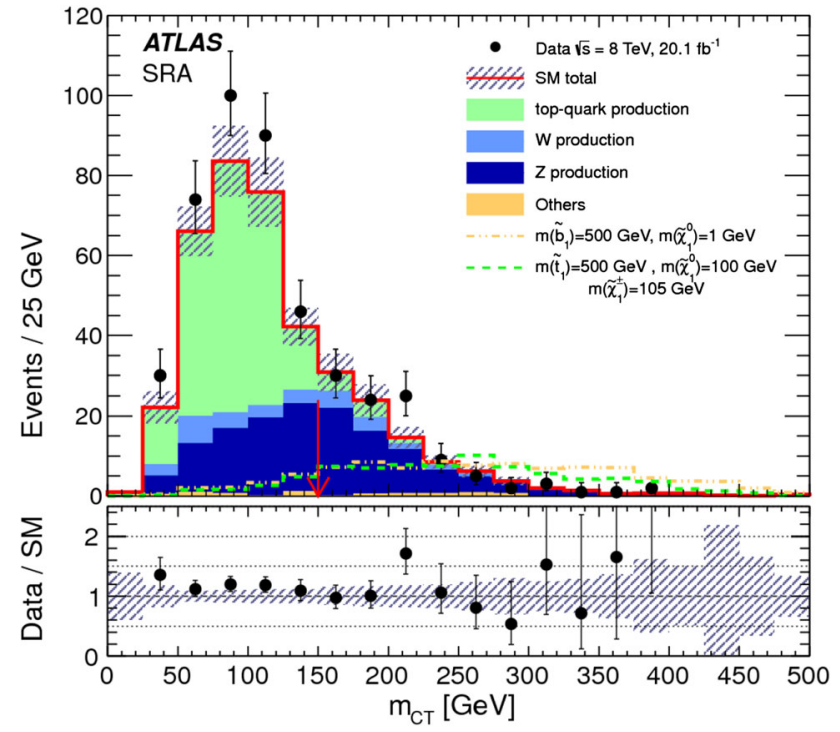

Fig. 22 The $m_{\mathrm{CT}}$ distribution with all selection criteria applied except for the $m_{\mathrm{CT}}$ thresholds [86]

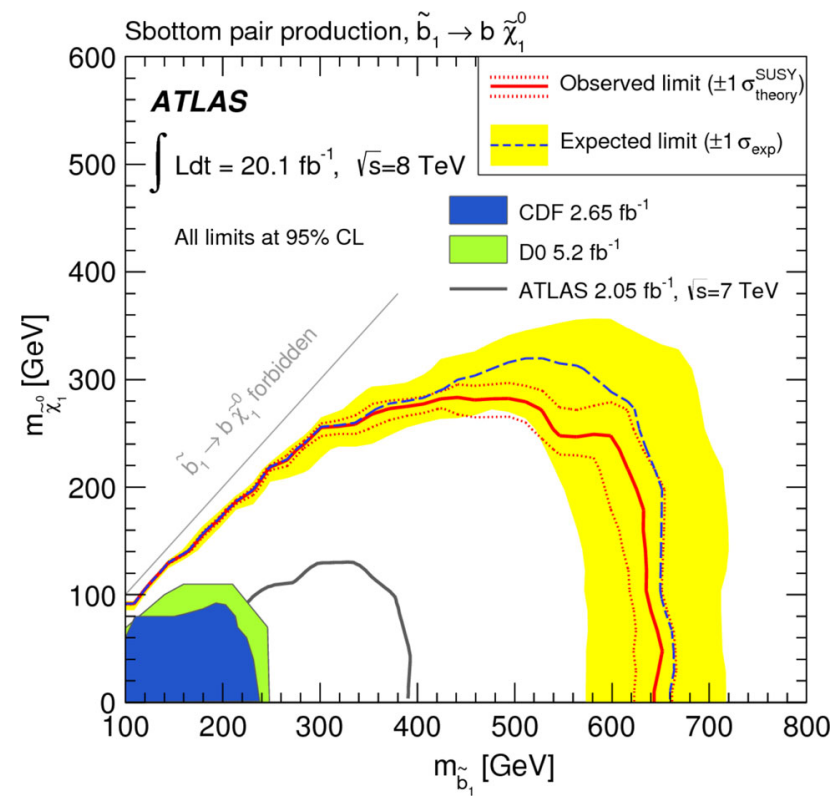

Fig. 23 Exclusion limits at $95 \% \mathrm{CL}$ on the bottom squark pair production in the bottom squark-LSP mass plane for a simplified model assuming $\widetilde{b} \rightarrow b \widetilde{\chi}_{1}^{0}[86]$. All other sparticles are decoupled

branching fraction of $60 \%$ and no possibility to detect other decay chains are assumed, the limit drops to $m_{\widetilde{\mathrm{b}}}<520 \mathrm{GeV}$ and $m_{\widetilde{\chi}_{1}^{0}}<150 \mathrm{GeV}$. Note that this search is also sensitive to the direct production of top squarks with subsequent decay to $\tilde{\mathrm{t}} \rightarrow \mathrm{b} \tilde{\chi}_{1}^{ \pm}$, with $\tilde{\chi}_{1}^{ \pm} \rightarrow \mathrm{W}^{ \pm} \widetilde{\chi}_{1}^{0}$. The sensitivity of this search is comparable to the one-lepton search discussed above for small chargino-neutralino mass differences of a few $\mathrm{GeV}$, where the leptons are too soft to be detected.

The results of the same-sign dilepton search [74], discussed in Sect. 4.1.2, can also be interpreted in terms of 
a direct bottom squark search, where the bottom squarks are pair-produced and then each decay as $\widetilde{\mathrm{b}} \rightarrow \mathrm{t} \tilde{\chi}_{1}^{-}$, with $\tilde{\chi}_{1}^{-} \rightarrow \mathrm{W}^{-} \tilde{\chi}_{1}^{0}$ (and charge conjugate, respectively). Here, same-sign dileptons can originate from leptonic top-quark and $\mathrm{W}$-boson decays. Bottom squark masses below $500 \mathrm{GeV}$ can be excluded for chargino masses up to $350 \mathrm{GeV}$ and neutralino masses up to $150 \mathrm{GeV}$, if $m_{\widetilde{\chi}_{1}^{0}} / m_{\widetilde{\chi}_{1}^{ \pm}}=0.5$. Slightly higher neutralino masses, up to $180 \mathrm{GeV}$ can be excluded for $m_{\widetilde{\chi}_{1}^{0}} / m_{\widetilde{\chi}_{1}^{ \pm}}=0.8$.

In summary, directly produced bottom squark have been searched for the first time in the region predicted by naturalness. Bottom squark masses below $600 \mathrm{GeV}$ up to $\widetilde{\chi}_{1}^{0}$ masses of $250 \mathrm{GeV}$ can be excluded assuming large branching fractions to the examined final states.

\subsection{SUSY models with $\widetilde{\mathrm{G}}$ as LSP}

GMSB models where only the $\widetilde{t}_{R}$ and the higgsinos are accessible [87] provide a perfect example of a natural scenario. The lightest chargino and the two lighter neutralinos are almost pure higgsinos and therefore nearly mass degenerate, which corresponds to scenario (c) of Fig. 2. The following decay is therefore considered: $\widetilde{\mathrm{t}}_{\mathrm{R}} \rightarrow \widetilde{\chi}_{1}^{+} \mathrm{b} \rightarrow f f^{\prime} \tilde{\chi}_{1}^{0} \mathrm{~b} \rightarrow f f^{\prime} \mathrm{h}^{0}(\rightarrow$ $\gamma \gamma, \mathrm{b} \overline{\mathrm{b}}) \widetilde{\mathrm{G}} \mathrm{b}$ where $\mathrm{f}$ and $\mathrm{f}^{\prime}$ are low-energetic quarks or leptons. SM background events are suppressed by requiring the invariant mass of two photons to be within the Higgs mass window, exploiting the sidebands for the background estimation. In addition, two b-tagged jets are required, originating from the top squark decay. As shown in Fig. 24, top squark masses below 360 to $410 \mathrm{GeV}$ are excluded, depending on the higgsino mass. Note that the direct $\tilde{\chi}_{1}^{+} \tilde{\chi}_{1}^{-}$production channel can have a similar final state when b-tagged jets are coming from one of the $\mathrm{h}^{0}$. Considering this decay therefore increases the sensitivity for low $\widetilde{\chi}_{1}^{0}$ mass and top squark mass above $300 \mathrm{GeV}$, where the $\tilde{\chi}_{1}^{+} \widetilde{\chi}_{1}^{-}$production cross section dominates over direct top squark production.

For the top squark decay considered, other final states can occur as well. First the two Higgs bosons could decay as $\mathrm{h}^{0} \rightarrow \mathrm{ZZ} \rightarrow 1111$ instead of $\gamma \gamma / \mathrm{b} \overline{\mathrm{b}}$. Second the lightest neutralino could also decay as $\widetilde{\chi}_{1}^{0} \rightarrow \mathrm{Z}(\rightarrow 11) \widetilde{\mathrm{G}}$ giving $\mathrm{Zh}^{0}$ or even ZZ final states. In all of these cases, the multi-lepton analysis [81], described in Sect. 4.2, is particularly sensitive. Models with a branching ratio of $100 \%$ for $\widetilde{\chi}_{1}^{0} \rightarrow Z \widetilde{\mathrm{G}}$ and a branching ratio of $50 \%$ for each $\widetilde{\chi}_{1}^{0}$ decay are considered. As shown in Fig. 25, for a branching ratio of $100 \%$ to $\mathrm{Z}$ bosons and $\widetilde{\mathrm{G}}$, top squark masses below $510 \mathrm{GeV}$ can be excluded for $\tilde{\chi}_{1}^{ \pm}$masses of up to $450 \mathrm{GeV}$. The limits for the other two cases are weaker.

In summary, the mass reach of GMSB top squark searches is about $200 \mathrm{GeV}$ weaker than for models with $\tilde{\chi}_{1}^{0}$ as LSP. No dedicated bottom squark search in the GMSB model is published yet, but a similar limit should be obtained since the

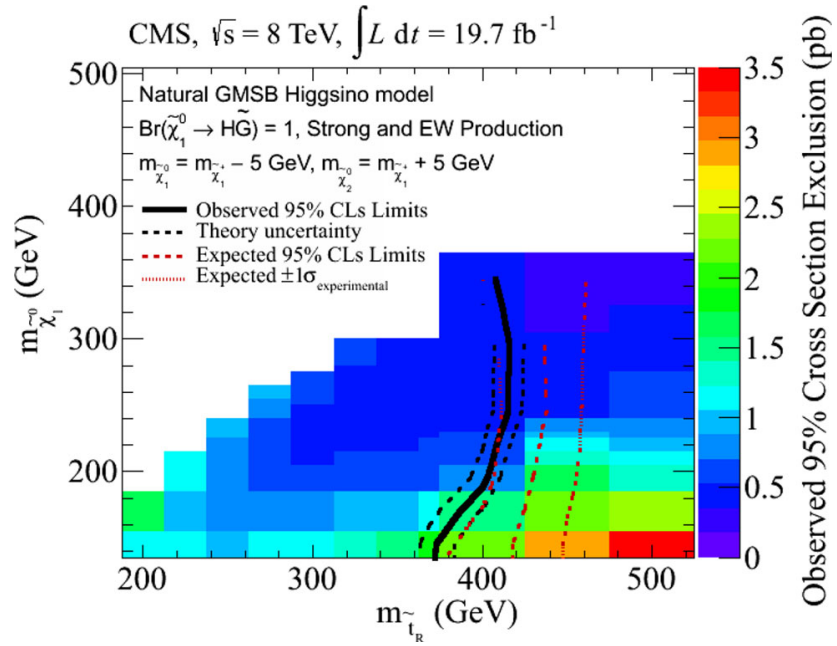

Fig. 24 Exclusion limits at $95 \% \mathrm{CL}$ on the top squark pair production in the top squark-LSP mass plane for a simplified model assuming $\widetilde{\mathfrak{t}}_{\mathrm{R}} \rightarrow \tilde{\chi}_{1}^{+} \mathrm{b}$. Further assumptions are that $\widetilde{\chi}_{1}^{+}$is higgsino-like, $\widetilde{\chi}_{1}^{0}$ is the NLSP decaying as $\widetilde{\chi}_{1}^{0} \rightarrow \mathrm{h}^{0} \widetilde{\mathrm{G}}$ and $\widetilde{\mathrm{G}}$ is the LSP [87]

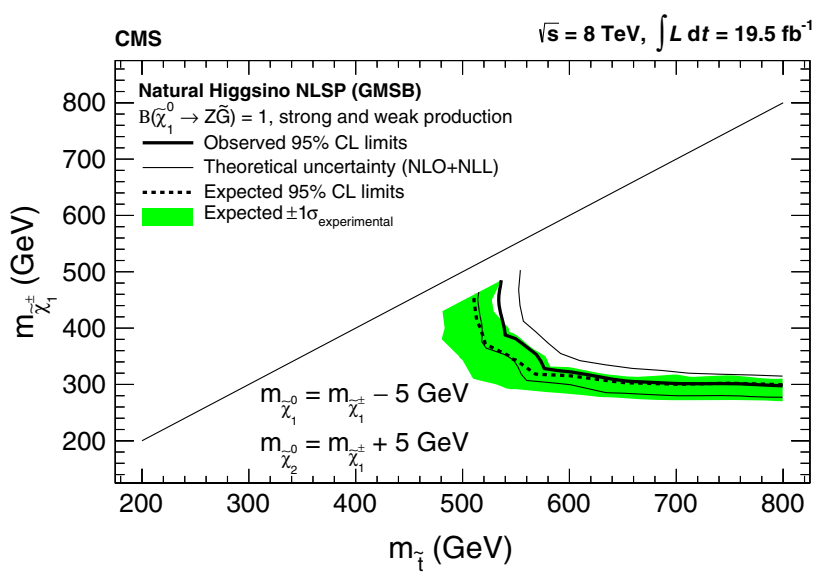

Fig. 25 Exclusion limits at $95 \% \mathrm{CL}$ on the top squark pair production in the top squark-LSP mass plane for a simplified model assuming $\tilde{\mathrm{t}} \rightarrow \widetilde{\chi}_{1}^{+}$b. Further assumptions are that $\widetilde{\chi}_{1}^{+}$is higgsino-like, $\widetilde{\chi}_{1}^{0}$ is the NLSP decaying as $\widetilde{\chi}_{1}^{0} \rightarrow Z \widetilde{G}$ and $\widetilde{\mathrm{G}}$ is the LSP [81]

final-state $\widetilde{\mathrm{b}}_{1} \rightarrow \widetilde{\chi}_{1}^{0} \mathrm{~b}$ is very similar to that of the top squark $\left.\tilde{\mathrm{t}}_{1} \rightarrow \tilde{\chi}_{1}^{+} \mathrm{b} \rightarrow f f^{\prime} \tilde{\chi}_{1}^{0}\right)$ when the low-energetic fermions $\mathrm{f}$ and $\mathrm{f}^{\prime}$ are not reconstructed.

\section{Electroweak SUSY sector}

In natural SUSY, many weakly interacting particles are expected to be close to the EW scale. Searches for neutral and charged Higgses with positive $R$-parity are presented in Sect. 6.1. Searches for partners of the Higgses and electroweak gauge bosons, called electroweakinos (EWKinos), are discussed in Sect. 6.2. Finally, the limits on the sleptons are discussed in Sect. 6.3. 


\subsection{SUSY Higgses}

As already mentioned in Sect. 2, the Higgs boson discovered is assumed to be the lightest neutral Higgs of the $\operatorname{MSSM}\left(\mathrm{h}^{0}\right)$. It is worth to note that a value of $126 \mathrm{GeV}$ is close to the upper mass bound possible for $\mathrm{h}^{0}$ in MSSM and requires $\mathrm{O}(1 \mathrm{TeV})$ top squark mass or a fine-tuned value of top squark mixing. This creates a tension with the natural SUSY spectrum where the top squark mass should be $600 \mathrm{GeV}$ maximum. Results of searches are extensively discussed in [9] of this review. This section therefore proposes only a short summary.

Extra neutral and charged Higgses, which preferentially couple to the most massive down-type fermions, are actively searched. At tree level, their masses only depend on $\tan \beta$ and $m_{\mathrm{A}^{0}} .{ }^{9}$ At LHC, neutral Higgses are produced singly or accompanied by b-jet(s) and decay via $\tau^{+} \tau^{-}, \mathrm{b} \overline{\mathrm{b}}$ and more marginally $\mu^{+} \mu^{-}$final states. Charged Higgses with lower masses than the top quark will predominantly appear in the top decay via $\mathrm{t} \rightarrow \mathrm{bH}^{ \pm}$. When charged Higgses have higher masses than the top quark, they will be produced in association with top and bottom quarks. In both cases, they mainly decay via $\mathrm{H}^{ \pm} \rightarrow \tau^{ \pm} \nu$. Results from searches favor neutral and charged SUSY Higgses with masses higher than $\mathrm{h}^{0}$, even if no model-independent limits exist yet.

It is worth to mention that more intricate searches are also investigated. For example, searches for a topology in which a $\mathrm{H}^{0}$ decays via a cascade of lighter charged and neutral Higgs bosons ${ }^{10}$ have been performed by ATLAS [88].

\subsection{EWKinos}

As discussed in Sect. 2, neutralino and chargino masses are obtained by mixing gauge eigenstates. The sensitivity to the three typical scenarios shown in Fig. 2 and corresponding to (a) bino-like, (b) wino-like, and (c) higgsino-like $\widetilde{\chi}_{1}^{0}$ are now reviewed both for $\tilde{\chi}_{1}^{0}$ (Sect. 6.2.1) and the gravitino (Sect. 6.2.2) being the LSP.

\subsubsection{SUSY models with $\tilde{\chi}_{1}^{0}$ as LSP}

At LHC, most efforts concentrate on processes involving the two lightest neutralinos $\left(\widetilde{\chi}_{1}^{0}, \widetilde{\chi}_{2}^{0}\right)$ and the lightest chargino $\left(\widetilde{\chi}_{1}^{ \pm}\right)$. Assuming that the EWKinos are the lightest sparticles of the spectrum (Fig. 3), the main production occurs via the s-channel exchange of a virtual gauge boson. EWKinos then naturally decay as $\widetilde{\chi}_{2}^{0} \rightarrow \mathrm{Z} / \mathrm{h}^{0(*)} \widetilde{\chi}_{1}^{0}$ and $\tilde{\chi}_{1}^{ \pm} \rightarrow \mathrm{W}^{(*)} \widetilde{\chi}_{1}^{0}$. Given the low values of cross sections compared to SM backgrounds, searches are conducted most of the times for lep-

\footnotetext{
${ }^{9}$ For charged Higgses $m_{\mathrm{H}^{ \pm}}^{2}=m_{\mathrm{A}}^{2}+m_{\mathrm{W}}^{2}$ at tree level. Other SUSY parameters enter via radiative corrections and are fixed to particular benchmark values, chosen to exhibit certain MSSM features.

${ }^{10} \mathrm{H}^{0} \rightarrow \mathrm{W}^{\mp} \mathrm{H}^{ \pm} \rightarrow \mathrm{W}^{\mp} \mathrm{W}^{ \pm} \mathrm{h}^{0} \rightarrow \mathrm{W}^{\mp} \mathrm{W}^{ \pm} \mathrm{b} \overline{\mathrm{b}}$
}

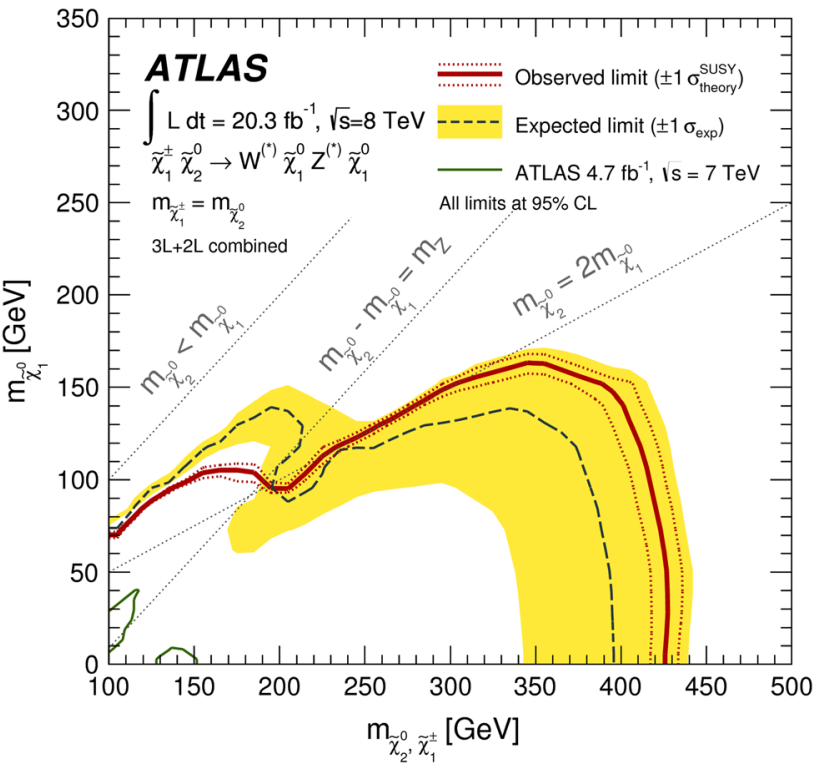

Fig. 26 Exclusion limits at $95 \% \mathrm{CL}$ on the $\tilde{\chi}_{1}^{ \pm} \tilde{\chi}_{2}^{0}$ production in the $\tilde{\chi}_{1}^{ \pm}$-LSP mass plane [76] for a simplified model assuming $\tilde{\chi}_{1}^{ \pm}$and $\tilde{\chi}_{2}^{0}$ are mass degenerate and decay via $\tilde{\chi}_{1}^{ \pm} \rightarrow W^{ \pm(*)} \tilde{\chi}_{1}^{0}$ and $\tilde{\chi}_{2}^{0} \rightarrow Z^{0(*)} \tilde{\chi}_{1}^{0}$. All other SUSY particles are decoupled

tonic decays of $\mathrm{Z}$ and $\mathrm{W}$, giving $1-4$ leptons $+E_{\mathrm{T}}^{\text {miss }}$ final states. Note that an excess in these channels could well be the only SUSY signal at LHC if colored sparticles are too heavy or decay through intricate chains.

At LHC, scenario (a) with bino-like $\tilde{\chi}_{1}^{0}$ and winodominated $\widetilde{\chi}_{2}^{0}$ and $\widetilde{\chi}_{1}^{ \pm}$is the most favorable scenario, especially when the mass difference between $\tilde{\chi}_{2}^{0}$ and $\tilde{\chi}_{1}^{ \pm}$and the LSP allows for on-shell $\mathrm{Z}, \mathrm{h}^{0}$, and $\mathrm{W}$. The highest cross section is coming from $\tilde{\chi}_{1}^{ \pm} \widetilde{\chi}_{2}^{0}$ production, covered by a 3lepton $+E_{\mathrm{T}}^{\text {miss }}$ search. Most sensitive signal regions require the three leptons to be electrons or muons (the leading one should have $p_{\mathrm{T}}>25 \mathrm{GeV}$ ) and the invariant mass of the two same-flavor opposite-sign leptons $\left(m_{\mathrm{SFOS}}\right)$ to be close to the Z-boson mass. Further discrimination is obtained by selecting ranges of $E_{\mathrm{T}}^{\text {miss }}$ and $m_{\mathrm{T}}$ (formed with $E_{\mathrm{T}}^{\text {miss }}$ and the lepton not forming the SFOS lepton pair). The search sensitivity is driven by the ability to reduce and control the WZ background. Assuming mass degeneracy between $\tilde{\chi}_{1}^{ \pm}$and $\tilde{\chi}_{2}^{0}$, stringent limits are obtained on EWKinos: $m_{\widetilde{\chi}_{2}^{0}, \widetilde{\chi}_{1}^{ \pm}<}<$ $340 \mathrm{GeV}$ for LSP masses lower than $70 \mathrm{GeV}$ are excluded [89]. ${ }^{11}$ Recent efforts were made to cover the case where $\mathrm{Z}$ and/or $\mathrm{W}$ are not on-shell and where $\mathrm{h}^{0}$ is present in the decay. This is done by considering bins of $m_{\text {SFOS }}$ outside the $\mathrm{Z}$-mass and requiring the presence of tau-lepton(s). In this case the reducible background coming from jets or photons faking leptons is also of importance and could dominate over WZ, altering the sensitivity to this more compressed EWKino

\footnotetext{
$\overline{11}$ Note that with no mass degeneracy these upper bounds can be significantly lower.
} 
mass spectrum. However, together with the increase in luminosity and $\sqrt{s}$, this provides considerable improvement over $7 \mathrm{TeV}$ results, even if the compressed scenario case still has poor sensitivity.

The 3-lepton $+E_{\mathrm{T}}^{\text {miss }}$ final state is less favorable for scenario (a) where $M_{1} \ll \mu<M_{2}$, since the $\tilde{\chi}_{1}^{ \pm} \widetilde{\chi}_{2}^{0}$ production cross section is divided by 3 due to the lower coupling of the higgsino to SM gauge bosons. Similarly scenario (b) suffers from the too large $\tilde{\chi}_{1}^{ \pm}-\widetilde{\chi}_{2}^{0}$ mass difference. Finally, no sensitivity is expected from scenario (c) because of the closeness of $\widetilde{\chi}_{1}^{ \pm}, \widetilde{\chi}_{2}^{0}$, and $\widetilde{\chi}_{1}^{0}$, resulting in too soft objects in the final state. To partially recover the sensitivity, other channels with 2-lepton $+E_{\mathrm{T}}^{\text {miss }}$ final state targeting the search for $\tilde{\chi}_{1}^{+} \tilde{\chi}_{1}^{-} \rightarrow \mathrm{W}^{+}\left(\rightarrow 1^{+} v\right) \mathrm{W}^{-}\left(\rightarrow 1^{-} v\right) \tilde{\chi}_{1}^{0} \tilde{\chi}_{1}^{0}$ and $\tilde{\chi}_{1}^{+} \tilde{\chi}_{2}^{0} \rightarrow$ $\mathrm{W}^{+}\left(\rightarrow \mathrm{qq}^{\prime}\right) \mathrm{Z}^{0}\left(\rightarrow 1^{-} 1^{+}\right) \tilde{\chi}_{1}^{0} \widetilde{\chi}_{1}^{0}$ are being developed [76]. In scenario (a) for massless $\widetilde{\chi}_{1}^{0}$, the former excludes chargino masses in the range $100<m_{\tilde{\chi}_{1}^{ \pm}}<160 \mathrm{GeV}$ while the latter extends further the $\tilde{\chi}_{1}^{ \pm} / \widetilde{\chi}_{2}^{0}$ mass limit to $410 \mathrm{GeV}$; see Fig. 26. Other modes like $\widetilde{\chi}_{1}^{+} \widetilde{\chi}_{1}^{0} \rightarrow \mathrm{W}^{+}\left(\rightarrow 1^{+} v\right) \widetilde{\chi}_{1}^{0} \widetilde{\chi}_{1}^{0}$ and $\tilde{\chi}_{2}^{0} \widetilde{\chi}_{1}^{0} \rightarrow Z^{0}\left(\rightarrow 1^{+} 1^{-}\right) \widetilde{\chi}_{1}^{0} \widetilde{\chi}_{1}^{0}$ are not yet explored due to the very low cross section and overwhelming inclusive $\mathrm{W}$ and $\mathrm{Z}$ cross section. Finally, even in the most favorable scenario (a), $\widetilde{\chi}_{1}^{0} \widetilde{\chi}_{1}^{0}$ and $\widetilde{\chi}_{2}^{0} \widetilde{\chi}_{2}^{0}$ productions are heavily suppressed at production level一 $\mathrm{O}(1 \mathrm{fb})$ for $100 \mathrm{GeV} \tilde{\chi}$ mass - and cannot be searched for at LHC even in dedicated monojet analyses $[90,91]$.

\subsubsection{SUSY models with $\widetilde{G}$ as LSP}

As for the strong production, new final states and search possibilities can emerge when the gravitino is the LSP. In scenario (a), the final state will contain two additional photons, reducing drastically the background from gauge bosons and therefore increasing the reach in mass. Reinterpreting the two 2-photons $+E_{\mathrm{T}}^{\mathrm{miss}}$ analysis described in Sect. 4.2, and considering $\tilde{\chi}_{1}^{ \pm} \tilde{\chi}_{1}^{0}$ production which have the highest cross section, exclude $m_{\tilde{\chi}_{1}^{ \pm}}<500 \mathrm{GeV}$ independently of the $\tilde{\chi}_{1}^{0}$ mass [77]. Similarly scenario (b) implies $\tilde{\chi}_{1}^{ \pm} \rightarrow \mathrm{W}^{+} \widetilde{\mathrm{G}}$ and $\tilde{\chi}_{1}^{0} \rightarrow \gamma / Z \widetilde{G}$ and it could be searched for in the 1-lepton $+1-$ photon channel, but no publication exists yet. Finally, scenario (c) is accessible thanks to the decay $\widetilde{\chi}_{1}^{0} \rightarrow \gamma / \mathrm{Z} / \mathrm{h}^{0} \widetilde{\mathrm{G}}$ where the branching ratios to $\gamma, \mathrm{Z}$, and $\mathrm{h}^{0}$ depend primarily on $\tan \beta$ and on the mass difference between $\widetilde{\chi}_{1}^{0}$ and $\widetilde{\mathrm{G}}$. In the case of Z-rich higgsino (low $\tan \beta$ value and positive $\mu$ ), final states with 4-leptons $+E_{\mathrm{T}}^{\text {miss }}$ or 2-leptons +2 -jets will provide interesting sensitivity, as shown in Fig. 27 for the 2011 LHC data [92]. Mixed $\mathrm{Z} / \mathrm{h}^{0}$ (higher $\tan \beta$ value) scenarios can be covered by 2 -leptons $+2 \mathrm{~b}+E_{\mathrm{T}}^{\text {miss }}$ final states. $\mathrm{h}^{0}$-rich higgsino scenarios (low $\tan \beta$ value and negative $\mu$ ) can be covered by $4 \mathrm{~b}+E_{\mathrm{T}}^{\text {miss }}$.

More complicated situations can occur beyond the three scenarios discussed in Fig. 2. For example, if $M_{1}$ and $\mu$ are

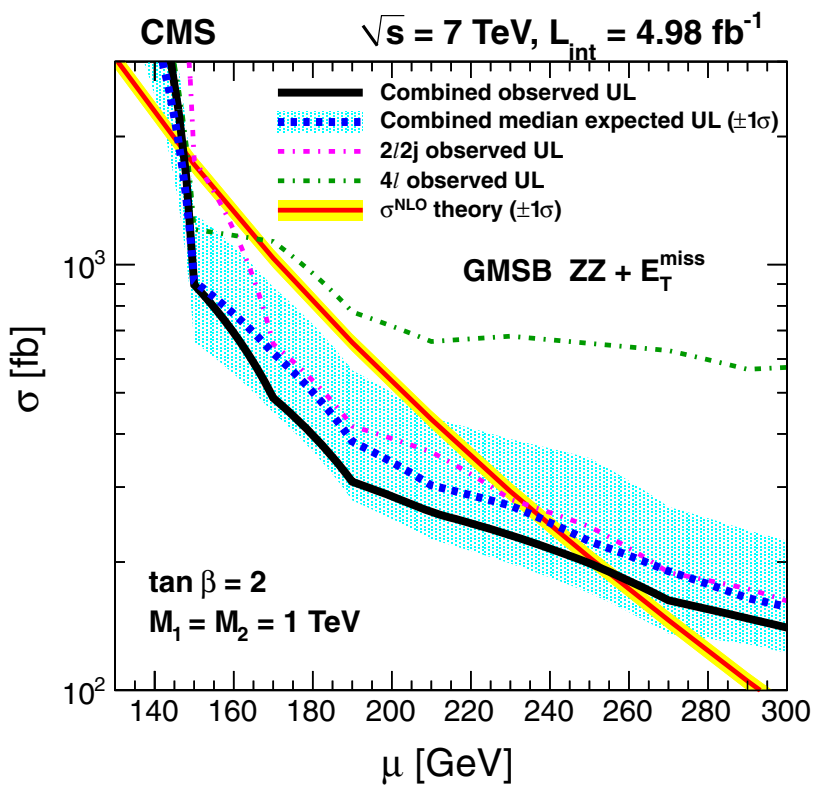

Fig. 27 Exclusion limits at $95 \% \mathrm{CL}$ on the production cross section of higgsino-like EWKinos $\widetilde{\chi}_{1}^{0}, \widetilde{\chi}_{2}^{0}, \widetilde{\chi}_{1}^{ \pm}$. The decay of the NLSP $\widetilde{\chi}_{1}^{0} \rightarrow$ $\mathrm{Z}^{0(*)} \widetilde{\mathrm{G}}$ is forced and all other SUSY particles are decoupled [92]

approximately equal and the NLSP is a bino/higgsino admixture, large branching ratios to photons and Higgs bosons are generated. The $1 \mathrm{~b}+$ photon $+E_{\mathrm{T}}^{\text {miss }}$ final state, described in Sect. 4.2, can exclude $\widetilde{\chi}_{1}^{0}$ masses between 200 and $400 \mathrm{GeV}$, see Fig. 18.

In conclusion, EWKino searches provide presently much weaker constraints on the natural SUSY scenario than the strong production searches. Limits are still model-dependent and rely on many assumptions. More results are still expected in the near future, and ultimately the limits should be set in the pMSSM to understand better how complete the current searches are. Given that, it is fair to say that a complete exploration of this sector is still to come and will greatly benefit from the high luminosity program of LHC.

\subsection{Sleptons}

Sleptons $(\widetilde{\mathrm{e}}, \tilde{\mu}, \widetilde{\tau}$, and $\widetilde{v}$ ) are governed by five parameters: masses of the left-handed and right-handed $\widetilde{e} / \tilde{\mu}$, which are assumed to be mass degenerate in the MSSM, masses of the left-handed and right-handed staus and the stau mixing angle. $\widetilde{v}$ masses can be related to the charged slepton parameters. From naturalness arguments $\mathrm{O}(1 \mathrm{TeV})$ slepton masses are expected: the very low slepton production cross section, see Fig. 4, will therefore prevent their discovery. However, searching for $\mathrm{O}(500 \mathrm{GeV})$ sleptons could be achievable with high luminosity. The reason is that these very low cross sections with respect to EWKino production, are largely recovered by the more favorable branching ratio $\mathrm{BR}\left(\widetilde{1}^{+} \rightarrow 1^{+} \widetilde{\chi}_{1}^{0}\right)=100 \%$ compared to leptonic branching 


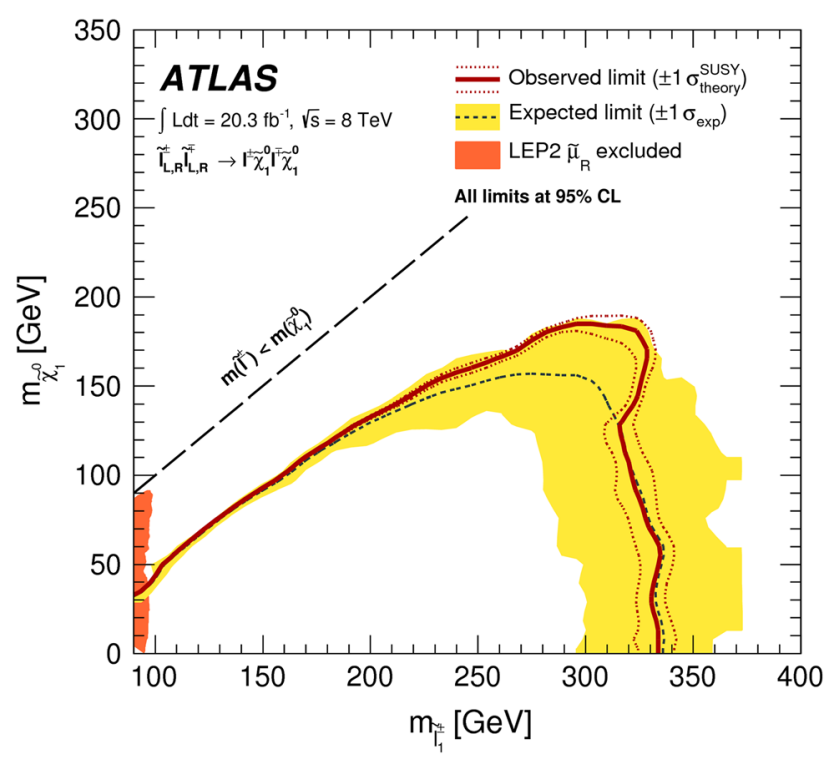

Fig. 28 Exclusion limits at $95 \% \mathrm{CL}$ on the slepton-slepton production in the slepton-LSP mass plane. The slepton decay $\widetilde{1} \rightarrow 1 \widetilde{\chi}_{1}^{0}$ is forced and all other SUSY particles are decoupled [93]

ratios of $\mathrm{W}$ and $\mathrm{Z}$. Lepton searches rely almost entirely on the very powerful discriminant variables like $m_{\mathrm{T} 2}$ or $m_{\mathrm{CT}}$ and electron(s) or muon(s) in the final states (presently no sensitivity is obtained for staus). Like in the direct bottom squark search (Fig. 22), the edge of slepton events with $m_{\tilde{l}}-m_{\widetilde{\chi}_{1}^{0}}>\mathrm{O}(100 \mathrm{GeV})$ appears far above the top and WW background ones.

The most promising final state is 2-lepton $+E_{\mathrm{T}}^{\mathrm{miss}}$, coming from $\tilde{1}^{+} \tilde{1}^{-} \rightarrow 1 \bar{l} \widetilde{\chi}_{1}^{0} \widetilde{\chi}_{1}^{0}$. Mass degenerate left- and righthanded selectrons and smuons are excluded below $325 \mathrm{GeV}$ masses for massless neutralinos, largely exceeding the LEP limit for right-handed smuons as shown in Fig. 28 [93]. Upper limits for left- and right-handed slepton masses are also obtained and give $250 \mathrm{GeV}$ and $300 \mathrm{GeV}$ for massless neutralinos.

Direct slepton production can also be accessed when the gravitino is the LSP. Various final states can be considered depending on the nature of the NSLP generating final states very similar to the EWKino searches. A particular interesting one corresponds to the NSLP slepton scenario where righthanded $\widetilde{e} / \widetilde{\mu}$ decay to an electron/muon and a right-handed $\tilde{\tau}_{\mathrm{R}}$ gives a tau and a gravitino. This generates a multi-lepton final state where the lepton multiplicity depends on the tau decay [81]. $\widetilde{\tau}_{\mathrm{R}}$ masses can then be excluded up to $200 \mathrm{GeV}$.

To be complete, a very favorable situation arises if the sleptons are interleaved between $\widetilde{\chi}_{2}^{0} / \widetilde{\chi}_{1}^{ \pm}$and $\widetilde{\chi}_{1}^{0}$ in scenario (a). Two cases can be envisaged: assume that all sleptons are mass degenerate (a1) and consider that only $\tilde{\tau}$ and $\widetilde{v_{\tau}}$ are light (a2). In both cases, the EWKinos will couple to the sleptons generating 2-leptons $+E_{\mathrm{T}}^{\text {miss }}$ and 3-lepton $+E_{\mathrm{T}}^{\text {miss }}$ final states with higher $\sigma \times$ BR than in the direct slepton or direct EWKino case discussed previously in this section. If the slepton masses are exactly in between $\widetilde{\chi}_{2}^{0} / \widetilde{\chi}_{1}^{ \pm}$and $\widetilde{\chi}_{1}^{0}$, mass limits reached in Fig. 26 are increased by a factor 2 to 3 for scenario (a1) and remain similar for scenario (a2) [89].

\section{Escape routes: $R$-parity violation, long-lived particle searches and beyond MSSM signatures}

Given the absence of signal from plain vanilla MSSM signatures, it is of paramount importance to look at scenarios where $R$-parity is violated (Sect. 7.1) and/or sparticle decays are not prompt (Sect. 7.2). In both cases, the stringent limits discussed in Sects. 4, 5 and 6 generally do not apply. Pushing further this idea, sensitivity to signatures appearing in scenarios beyond MSSM are also discussed in Sect. 7.3.

\section{$7.1 R$-parity violation searches}

$R$-parity conservation implies pair production of the superpartners and requires the lightest superpartners to be stable, leading to typical missing transverse energy signatures in the detector. If $R$-parity is not conserved these constraints do not exist anymore and dedicated searches need to be performed.

In RPV scenarios, the current limits of the proton decay can be met if only either $B$ or $L$ (and not both) is violated and the violation is sufficiently small [94]. Such models can also accommodate non-zero neutrino masses and neutrino oscillations. The RPV superpotential $W_{\mathrm{RPV}}$ includes three trilinear terms parameterized by the 48 Yukawa couplings $\lambda_{i j k}, \lambda_{i j k}^{\prime}, \lambda_{i j k}^{\prime \prime}$

$W_{\mathrm{RPV}}=\frac{1}{2} \lambda_{i j k} L_{i} L_{j} \bar{E}_{k}+\lambda_{i j k}^{\prime} L_{i} Q_{j} \bar{D}_{k}+\lambda_{i j k}^{\prime \prime} \bar{U}_{i} \bar{D}_{j} \bar{D}_{k}$,

where $i, j, k$ are generation indices, $L$ and $Q$ the $S U(2)_{L}$ doublet superfields of the leptons and quarks, and $\bar{E}, \bar{D}$ and $\bar{U}$ the $S U(2)_{L}$ singlet superfields of the charged leptons and the up- and down-type quarks.

The nature of the LSP - which is neutral and colorless in $R$-parity conserving models-might be different in RPV models and might be charged and/or carry color as well.

Searches for models with leptonic RPV interactions $\left(\lambda_{i j k} \neq 0\right.$ or $\left.\lambda_{i j k}^{\prime} \neq 0\right)$ are discussed in Sects. 7.1.1 and 7.1.2, respectively, while the quark RPV interactions $\left(\lambda_{i j k}^{\prime \prime} \neq 0\right)$ are reviewed in Sect. 7.1.3. Because of present constraints on RPV couplings, the values considered are generally in the range $\mathrm{O}\left(10^{-2}-10^{-5}\right)$. If the phase space for the LSP decay is very small $\left(\lambda<10^{-5}\right)$, it might also be long-lived. Such cases are covered in Sect. 7.2.

\subsubsection{Search for leptonic RPV interactions $\left(\lambda_{i j k} \neq 0\right)$}

With leptonic RPV interactions, multi-lepton final states are expected, which is particularly favorable at LHC where the 


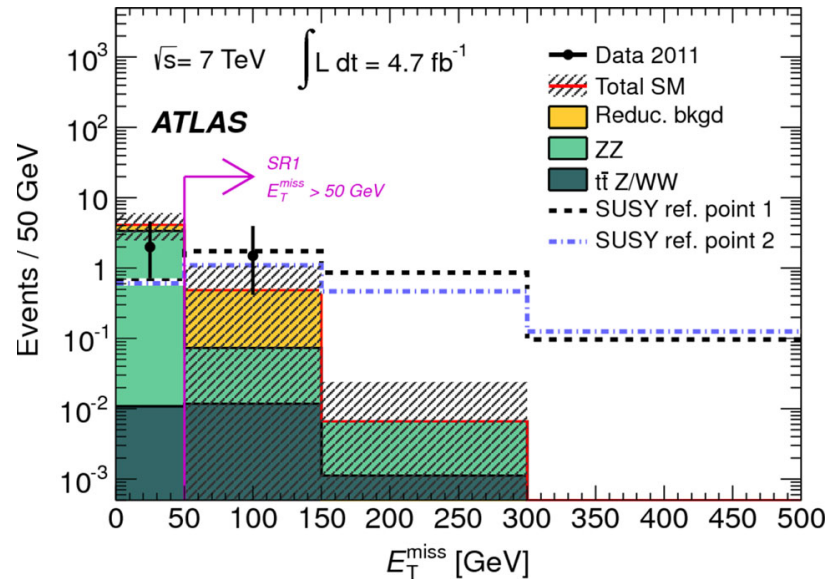

Fig. $29 E_{\mathrm{T}}^{\mathrm{miss}}$ distribution for events with at least four leptons and no Z-boson candidates. 'SUSY ref. point 1' is a simplified model point defined by $\mathrm{m}_{\tilde{\chi}_{1}^{ \pm}}=500 \mathrm{GeV}$ and $m_{\widetilde{\chi}_{1}^{0}}=300 \mathrm{GeV}$, while 'SUSY ref. point 2' is a MSUGRA/CMSSM model point defined by $m_{1 / 2}=$ $860 \mathrm{GeV}$ and $\tan \beta=37$ [95]

QCD background overwhelmingly dominates. The plethora of models considered is discussed in terms of the same order as for the RPC models, starting with strong production, then focusing on the third-generation, EWKino production and, finally, slepton production.

As a first example, a search with four or more leptons (electrons or muons) in the final state is discussed [95]. A non-zero coupling of $\lambda_{121}$ is chosen as a representative model. To veto low-energy resonances, the invariant mass of any opposite-sign same-flavor pair must be above $20 \mathrm{GeV}$ and outside a window around the Z-boson mass. Two signal regions according to different signal scenarios are defined. The first one requires $E_{\mathrm{T}}^{\text {miss }}>50 \mathrm{GeV}$ to be sensitive to models with missing energy originating from neutrinos. As an illustration, the $E_{\mathrm{T}}^{\text {miss }}$ distribution is displayed in Fig. 29. The other one is tuned to scenarios with a large multiplicity of high- $p_{\mathrm{T}}$ objects originating from heavier sparticles, by requiring $m_{\text {eff }}>300 \mathrm{GeV}$.

The strong production case is considered by looking at a full model, taken from Ref. [96], and tested in a MSUGRA/CMSSM parameter plane $\left(m_{1 / 2}, \tan \beta\right)$, for $m_{0}$, $A_{0}$ both zero, $\mu$ positive, and $\lambda_{121}=0.032$ at the unification scale. In this model, the $\widetilde{\tau}_{1}$ is the LSP and it decays through a virtual slepton or sneutrino as $\tilde{\tau}_{1} \rightarrow \tau \mathrm{e} \mu v_{\mathrm{e}}$ or $\tilde{\tau}_{1} \rightarrow \tau e e v_{\mu}$. Values of $m_{1 / 2}$ below $820 \mathrm{GeV}$ are excluded for $10<\tan \beta<40$. Note, however, that weak processes contribute to the SUSY pair production, dominating for $m_{1 / 2}>600 \mathrm{GeV}$. Therefore, a corresponding gluino mass below $1 \mathrm{TeV}$ is excluded in this model.

Multi-lepton final states can also arise when gravitino is the LSP and all right-handed sleptons are flavor degenerate - known as slepton co-NLSP scenario. Pair-produced gluinos and squarks eventually decay to the $\widetilde{\chi}_{1}^{0}$, which further decays

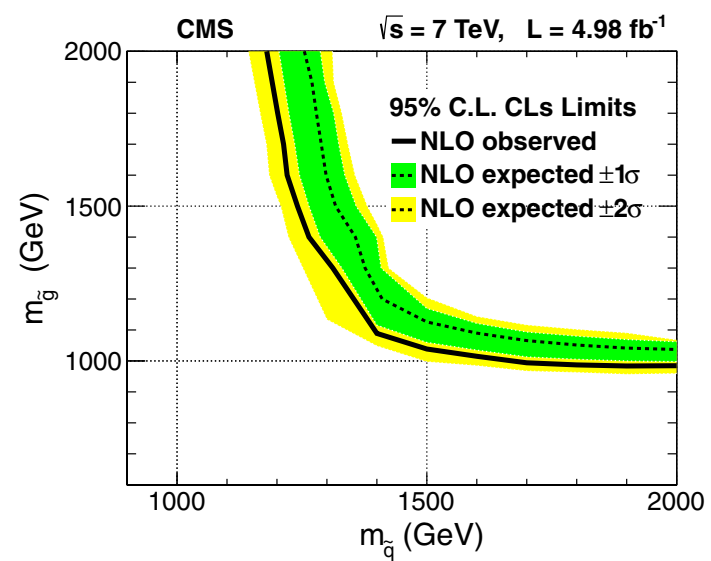

Fig. 30 Exclusion limits at $95 \% \mathrm{CL}$ in the squark and gluino mass plane for a GMSB RPV model with $\lambda_{123}=0.05$ [97]

to a slepton and a lepton, with the (right-handed) slepton decaying further to another lepton and the gravitino, yielding four leptons in the final state. Gluino masses below $1.2 \mathrm{TeV}$ and squark masses below $1 \mathrm{TeV}$ can be excluded with a $7 \mathrm{TeV}$ analysis [97], assuming an RPV coupling of $\lambda_{123}=0.05$, as shown in Fig. 30.

Leptonic RPV interactions can also arise in a search for direct top squark production [98], where each top squark decays to a top quark and an intermediate on- or off-shell bino which decays further through leptonic RPV interactions $\left(\lambda_{122}\right.$ or $\left.\lambda_{233}\right)$, via $\tilde{\chi}_{1}^{0 *} \rightarrow l_{i}+v_{j}+l_{k}$ or $\tilde{\chi}_{1}^{0 *} \rightarrow v_{i}+l_{j}+l_{k}$, where the indices $i, j, k$ refer to the ones in Eq. (3). The signature of direct top squark pair production with RPV decays is different from the one of RPC models, which implies a large amount of missing energy. In the RPV search, three or more isolated leptons (including hadronic $\tau$ candidates) and one or more b-tagged jets are required, but only low $E_{\mathrm{T}}^{\text {miss }}$. Instead $m_{\text {eff }}$ is used as discriminating variable.

The limits are extracted in the bino top squark mass plane, and found to be approximately independent of the bino mass. Top squark masses below $1020 \mathrm{GeV}$ can be excluded for bino masses of 200 to $1300 \mathrm{GeV}$ for a non-zero $\lambda_{122}$, and top squark masses below $820 \mathrm{GeV}$ for a non-zero $\lambda_{233}$.

The results of the 4-lepton $+E_{\mathrm{T}}^{\text {miss }}$ search [95], discussed at the beginning of this section, can also be interpreted in a simplified model where the lightest chargino and neutralino are the only sparticles with masses below the $\mathrm{TeV}$ scale. The pair-produced charginos decay each into a $\mathrm{W}$ boson and binolike $\widetilde{\chi}_{1}^{0}$ as in scenario (a) of Fig. 2. The LSP then decays through a virtual slepton or sneutrino as $\widetilde{\chi}_{1}^{0} \rightarrow \mathrm{e} \mu \nu_{\mathrm{e}}$ or $\widetilde{\chi}_{1}^{0} \rightarrow$ ee $v_{\mu}$ with a branching fraction of $50 \%$ each. The width of the $\widetilde{\chi}_{1}^{0}$ is fixed to $100 \mathrm{MeV}$ to ensure prompt decays. Choosing the best expected limit for each of the model points, with the $\sqrt{s}=7 \mathrm{TeV}$ data, chargino masses up to about $500 \mathrm{GeV}$ are excluded for LSP masses between 100 and $540 \mathrm{GeV}$ in the simplified model. 


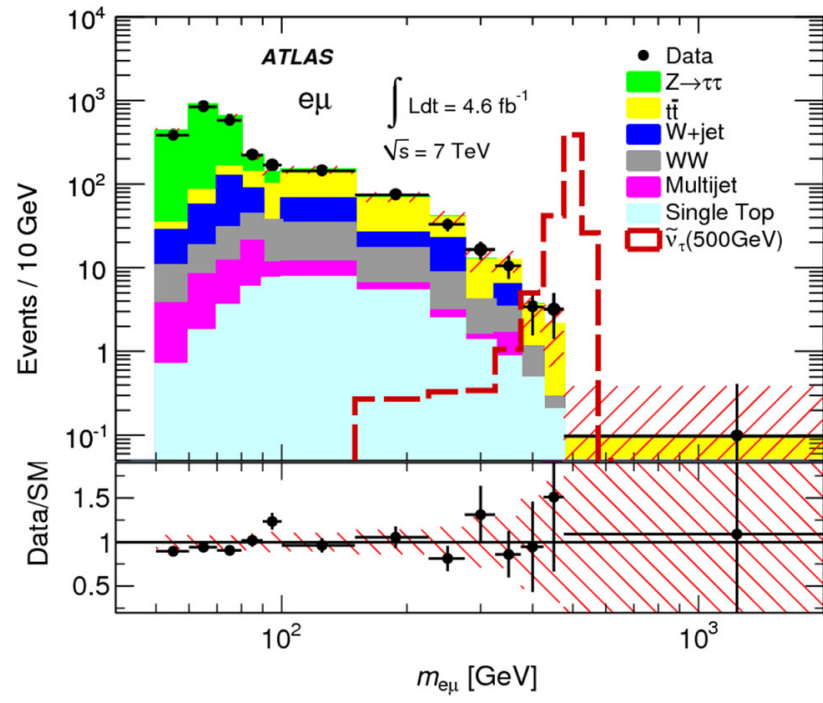

Fig. 31 Observed and predicted invariant e $\mu$-mass distribution. Signal simulations are shown for sneutrino mass of $500 \mathrm{GeV}, \lambda_{311}^{\prime}=0.11$, and $\lambda_{132}=0.07[99]$

In RPV SUSY, single sneutrinos can be produced via $\lambda_{311}^{\prime}$ coupling and then decay through $\lambda_{i j k}$ couplings to lepton pairs of different flavor. Searches for such scenarios have been performed in all possible combinations of differentflavor dilepton selections [99]. An example for a possible signal that is compatible with current exclusion limits on the strength of the RPV interactions from precision low-energy experiments [100], expectation, is given in Fig. 31 for the $\mathrm{e} \mu$ channel. Sneutrino masses of up to $1.6 \mathrm{TeV}$ are excluded in the e $\mu$ selection (for $\lambda_{311}^{\prime}=0.11$ and $\lambda_{132}=0.07$ ), where the mass resolution is better than in the channels including hadronically decaying tau leptons. The latter lead to sneutrino mass exclusion limits of the order of $1.1 \mathrm{TeV}$ for the same RPV interaction strength.

To summarize, as leptonic RPV interactions usually lead to signatures with many leptons, most scenarios are well covered with the current searches, and often result in sparticle mass limits that are stronger than those of RPC searches.

\subsubsection{Search for semi-leptonic $R P V$ interactions $\left(\lambda_{i j k}^{\prime} \neq 0\right)$}

Such signatures are specifically covered by the HERA experiments [101,102], which put stringent limits on the coupling between the first and second generation due to the nature of the unique $\mathrm{e}^{ \pm} \mathrm{p}$ accelerator. Therefore, LHC searches focus more on the third generation, as detailed below.

Signatures of models where the top squarks are light, while the other squarks and gluinos are decoupled, resemble those of third-generation leptoquarks. Trilinear RPV operators allow the lepton-number-violating decay $\tilde{\mathrm{t}}_{1} \rightarrow \tau \mathrm{b}$ with a coupling $\lambda_{333}^{\prime} \neq 0$, resulting in the same final state as from third-generation leptoquark decay, with similar kinematics.

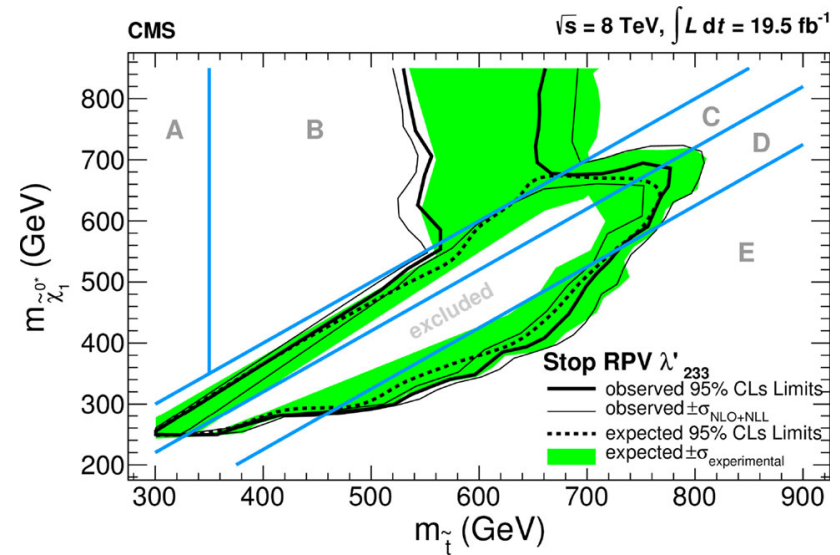

Fig. 32 Exclusion limits at $95 \%$ CL for RPV top squark decay in the top squark-LSP mass plane with non-zero $\lambda_{233}^{\prime}$ coupling. The region inside the curve is excluded. The different regions, A, B, C, D, and $\mathrm{E}$, mark different kinematic regions with different top squark decay products [98]

They can be tested by dedicated searches for $\tau$ and $\mathrm{b}$ quarks in the final state [103]. With $7 \mathrm{TeV}$ data, top squarks up to $525 \mathrm{GeV}$ are excluded assuming a simplified model with a branching ratio of $100 \%$ for $\widetilde{\mathfrak{t}}_{1} \rightarrow \tau \mathrm{b}$.

The top squark search [98], discussed in Sect. 7.1.1, can also be exploited for semileptonic RPV interactions $\left(\lambda_{233}^{\prime}\right)$, via $\widetilde{\chi}_{1}^{0 *} \rightarrow l_{i}+\mathrm{q}_{j}+\mathrm{q}_{k}$ or $\widetilde{\chi}_{1}^{0 *} \rightarrow v_{i}+\mathrm{q}_{j}+\mathrm{q}_{k}$, where the indices $i, j, k$ refer to the ones in Eq. (3). Limits are set for $\lambda_{233}^{\prime}$, where different kinematic regions lead to different allowed decays, ranging from two-body decays (for $m_{\mathrm{t}}+$ $m_{\widetilde{\chi}_{1}^{0}}<m_{\widetilde{\mathfrak{t}}_{1}}$ ) to four-body decays (e.g. for $m_{\widetilde{\chi}_{1}^{0}}>m_{\widetilde{\mathfrak{t}}_{1}}>$ $2 m_{\mathrm{t}}$ ). The resulting excluded region is shown in Fig. 32.

In summary, the signatures of semi-leptonic RPV interactions are similar to those expected in leptoquark decays. The LHC experiments can complement these searches with analyses including the third generation, where the most stringent limits up to now could be achieved.

\subsubsection{Search for quark RPV interactions $\left(\lambda_{i j k}^{\prime \prime} \neq 0\right)$}

The quark RPV interactions described by $\lambda_{i j k}^{\prime \prime}$ can be well tested with multi-jet resonance searches [104,105]. We here discuss an analysis searching for three-jet resonances [106], which tests two different RPV Yukawa couplings. While one search is inclusive, testing $\lambda_{122}^{\prime \prime}$, the other one requires at least one jet of each resonance decay to be b-tagged and is sensitive to $\lambda_{113}^{\prime \prime}$ and $\lambda_{223}^{\prime \prime}$. The jet-ensemble technique $[107,108]$ is used to combine the six highest- $p_{\mathrm{T}}$ jets into all possible unique triplets.

Limits are set on the gluino pair-production cross section times the branching fraction as a function of the gluino mass, as shown in Fig. 33. Gluinos with masses below $650 \mathrm{GeV}$ decaying to light-flavor jets can be excluded. Decays includ- 


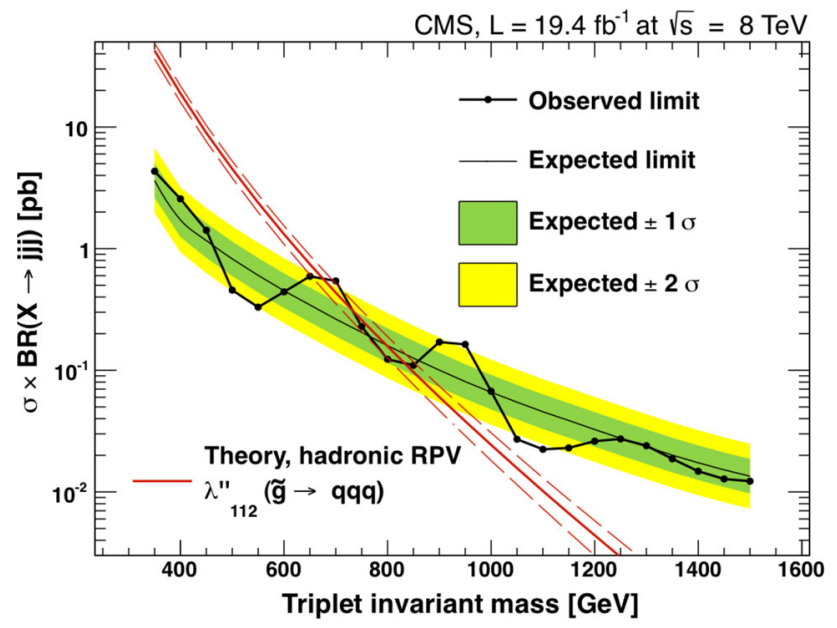

Fig. 33 Exclusion limits at $95 \% \mathrm{CL}$ on the production cross section of pair-produced gluinos for the inclusive RPV multi-jet search [106]

ing heavy-flavor jets can be excluded for even larger gluino masses, between 200 and $835 \mathrm{GeV}$.

Two other approaches exist. The first one counts the number of six-jets events above a given $p_{\mathrm{T}}$ threshold to search for high mass gluinos and the second one takes profit of the large boost of the low-mass gluinos [109]. With $7 \mathrm{TeV}$ data, gluino masses up to 666 and $255 \mathrm{GeV}$ can be excluded, respectively. The limit of the first approach is expected to reach $1 \mathrm{TeV}$ with $8 \mathrm{TeV}$ data.

Another scenario is given by a gluino decay to two quarks and one $\widetilde{\chi}_{1}^{0}$, which then further decays through a $\lambda_{i j k}^{\prime \prime}$ interaction to three quarks, leading to final states with ten jets when gluino pair production is assumed. A search for such scenarios is currently performed, but it has not yet been published.

The results of the same-sign dilepton search [74], discussed in Sect. 4.1.2, can also be interpreted in an RPV model, where gluinos are pair-produced and decay to three quarks via $\widetilde{\mathrm{g}} \rightarrow \operatorname{tbs}(\overline{\mathrm{t}} \overline{\mathrm{b}} \overline{\mathrm{s}})$, testing the $\lambda_{323}^{\prime \prime}$ coupling. In this decay $50 \%$ of the $\mathrm{W}$ bosons are expected to be same-sign, when both W bosons decay leptonically. As shown in Fig. 34, gluino masses up to $860 \mathrm{GeV}$ can be excluded.

In summary, a plethora of models with different RPV interactions exists, and those with the most striking signatures have been tested up to now, excluding a large phase space, but leaving holes for more complicated signatures still to be found with more data. This still leaves a large parameter space for not-yet-detectable RPV SUSY.

\subsection{Long-lived particle searches}

As no metastable particles are present in the Standard Model, long-lived particle searches are generally free of SM background. In turn, they require a deep understanding of the detector performance, which represents the only background,

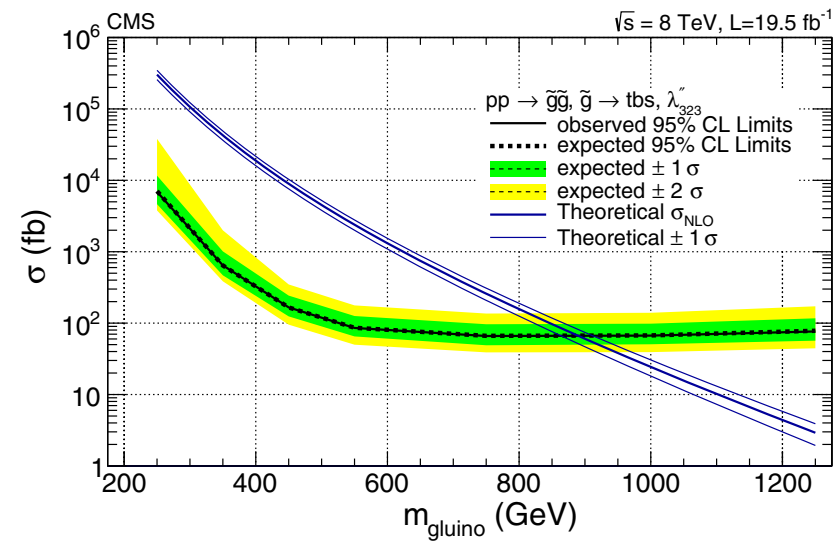

Fig. 34 Exclusion limits at $95 \% \mathrm{CL}$ on the production cross section of pair-produced gluinos for the same-sign dilepton analysis including heavy-flavor jets [74]

as discussed in Sect. 3.1.4. Metastable particles appear generally in the SUSY GUT theory framework [110-114]. They arise in three main situations: very low mass difference $(\leq \mathrm{O}(1 \mathrm{GeV}))$ between a SUSY particle and the LSP in RPC models, very weak $R$-parity violation, i.e. $\lambda, \lambda^{\prime}$ or $\lambda^{\prime \prime} \leq \mathrm{O}\left(10^{-5}\right)$, or very weak coupling to the gravitino in GMSB models. Depending on the SUSY mass spectrum, the metastable particle can be colored (squarks and gluinos) or not (sleptons, lightest chargino or neutralino). The experimental signatures probed at LHC are now reviewed by going from the left to the right of Fig. 6.

Non-pointing photons arise in GMSB models where the NLSP is the lightest neutralino with bino-like flavor, i.e. scenario (a) of Fig. 2. If the coupling strength with the gravitino is weak, the $\widetilde{\chi}_{1}^{0}$ lifetime is in the range $\mathrm{O}(0.1-100 \mathrm{~ns})$ accessible by the experiments, provided the $\widetilde{\chi}_{1}^{0}$ mass is close to the EW scale. In the EM calorimeter, non-pointing photons exhibit a singular geometric shape for the energy deposit and a late arrival. Results of the search are shown in Fig. 35 in the $\widetilde{\chi}_{1}^{0}$ lifetime-mass plane. ${ }^{12}$ The stronger limits obtained by ATLAS [116] for long lifetimes are explained by the standalone pointing capability of its calorimeter, whereas at short lifetimes CMS exploits better the correlation between $E_{\mathrm{T}}^{\text {miss }}$ and photon energy [117]. Non-pointing photons are not the only possibility in GMSB models. NLSP $\widetilde{\chi}_{1}^{0}$ will give nonpointing $\mathrm{Z}$ or Higgs. Slepton or squark/gluino NLSPs will give non-pointing leptons or jets. However, in all of these cases, no public results exist yet.

Reconstructing a displaced vertex with high mass and many tracks is undoubtedly a striking sign of new physics, as shown in Fig. 36. SUSY models with $\widetilde{\chi}_{1}^{0}$ LSP and very small RPV couplings can provide a plethora of possibilities: with leptons (electrons, muons or taus) attached to the

\footnotetext{
12 Weaker limits from converted photons reconstructed by the tracker as an electron-positron pair are not shown [115].
} 


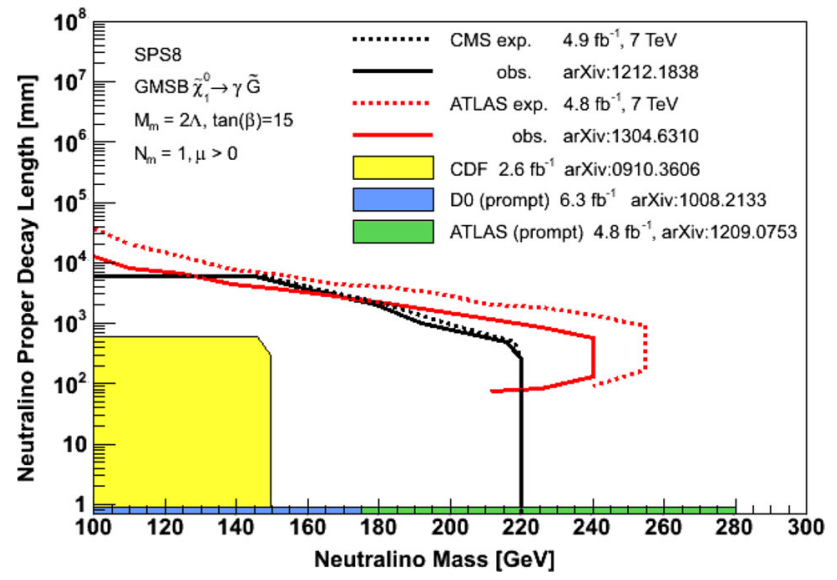

Fig. 35 Exclusion limits for non-pointing photon searches at $95 \% \mathrm{CL}$ in the mass-lifetime plane of the $\widetilde{\chi}_{1}^{0}$, assumed to be bino-like NLSP

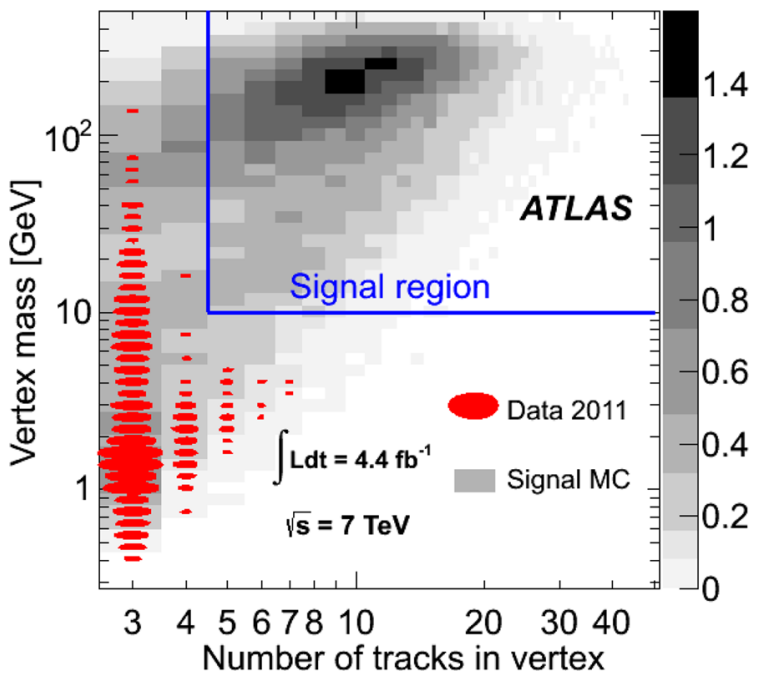

Fig. 36 Mass-track multiplicity plane of the displaced vertex selected by the ATLAS search. One muon is also asked to point to the displaced vertex. The SUSY model considered has $700 \mathrm{GeV}$ squarks, $500 \mathrm{GeV}$ $\tilde{\chi}_{1}^{0}$ and $\lambda_{2 i j}^{\prime}=0.3 \times 10^{-5}[118]$

displaced vertex or without leptons. While the latter case requires tracker and calorimeter information (cluster size, track multiplicity pointing to the cluster, etc.), the former relies on a dedicated tracking algorithm for non-standard displaced-vertex reconstruction. To date the only publicly available search interpreted in SUSY models is the one from ATLAS [118]. SUSY models with the simplified decay chain $\widetilde{\mathrm{q}} / \widetilde{\mathrm{g}} \rightarrow \mathrm{q} / \mathrm{q} \overline{\mathrm{q}}+\widetilde{\chi}_{1}^{0} \rightarrow \mathrm{q} / \mathrm{q} \overline{\mathrm{q}}+\mu \mathrm{q}_{i} \mathrm{q}_{j}$ are excluded for $\widetilde{\chi}_{1}^{0}$ lifetimes below $1 \mathrm{~m}$ and squark (gluino) masses below $0.7(0.9) \mathrm{TeV}$. Other searches looking for long-lived neutral bosons decaying in two leptons [119] could be useful to reject some SUSY models- though it is not yet done. In this case the displaced vertex is required to be at a distance of more than five standard deviations from the primary vertex in the transverse plane. Similar study is ongoing with two jets.

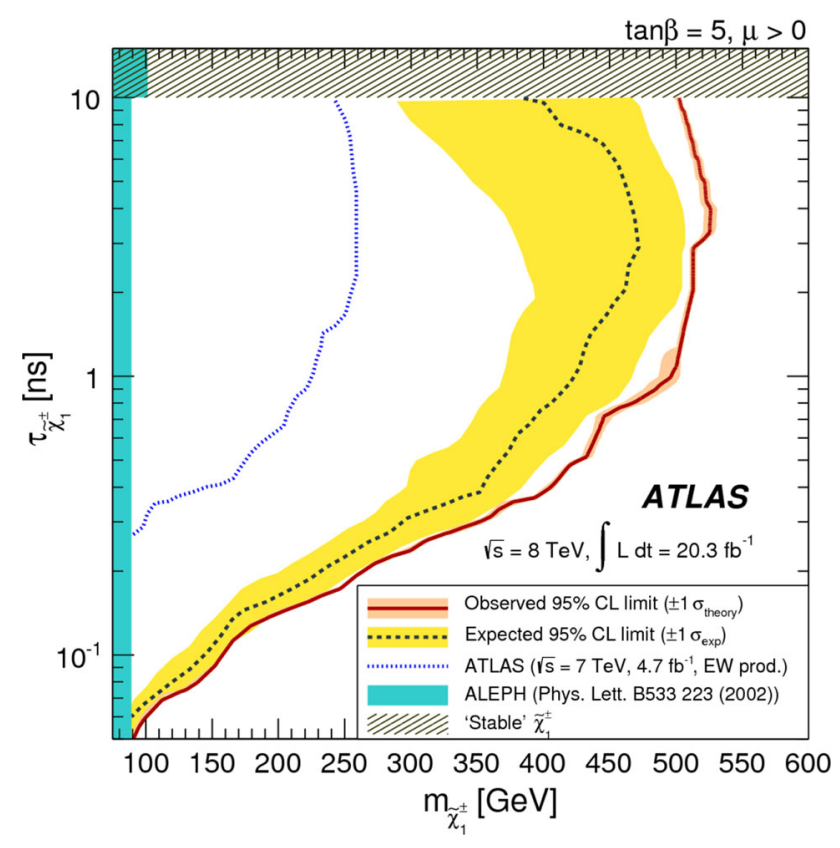

Fig. 37 Exclusion limit at $95 \%$ CL for disappearing track searches in mass-lifetime plane of the $\tilde{\chi}_{1}^{ \pm}[120]$

AMSB $[62,63]$ provides a well motivated case for metastable charginos, since $\tilde{\chi}_{1}^{ \pm}$and $\tilde{\chi}_{1}^{0}$ are almost degenerate and $m_{\widetilde{\chi}_{1}^{ \pm}}-m_{\widetilde{\chi}_{1}^{0}} \geq 140 \mathrm{MeV}$. The situation is similar to scenario (b) of Fig. 2. The chargino therefore decays after $\mathrm{O}(10 \mathrm{~cm})$ to undetectable particles, a soft pion and the LSP. This will cause the chargino track to 'disappear'. When produced directly $\left(\tilde{\chi}_{1}^{+} \tilde{\chi}_{1}^{-}, \tilde{\chi}_{1}^{ \pm} \tilde{\chi}_{1}^{0}\right)$ with an additional jet from initial-state radiation to trigger the event, one (or two) tracks may have no/few associated hits in the outer region of the tracking system. The continuous TRT tracking of the outer part of the ATLAS inner detector gives sensitivity to this signature. With the additional requirement of a high-energetic isolated track, regions beyond the LEP limits can be excluded in the lifetime-mass plane of the chargino, as shown in Fig. 37 [120]. Although originally motivated by AMSB, this result is largely model independent and fits also predictions in many 'unnatural' SUSY models [121-124], where the chargino and the LSP are the only accessible sparticles at LHC.

If the gluino and the LSP are almost mass degenerate, the gluino lifetime could be long enough for it to hadronize in $R$ hadrons ( $\widetilde{\mathrm{g} q} \overline{\mathrm{q}}, \widetilde{\mathrm{g}} \mathrm{qqq} \mathrm{q}^{\prime}$ ) or $R$-gluino balls ( $\left.\widetilde{\mathrm{g}} \mathrm{g}\right) .{ }^{13}$ These composite particles are detector-stable, highly ionizing, slowly moving (i.e. non-relativistic) and could change sign when they interact with the detector material. The signature can thus be a detector-stable charged particle, but also a charged particle turning neutral, or even a charged particle turning neutral and turning back charged. To fully explore all possibilities one needs to combine all possible detector mea-

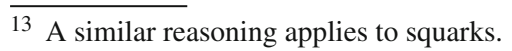




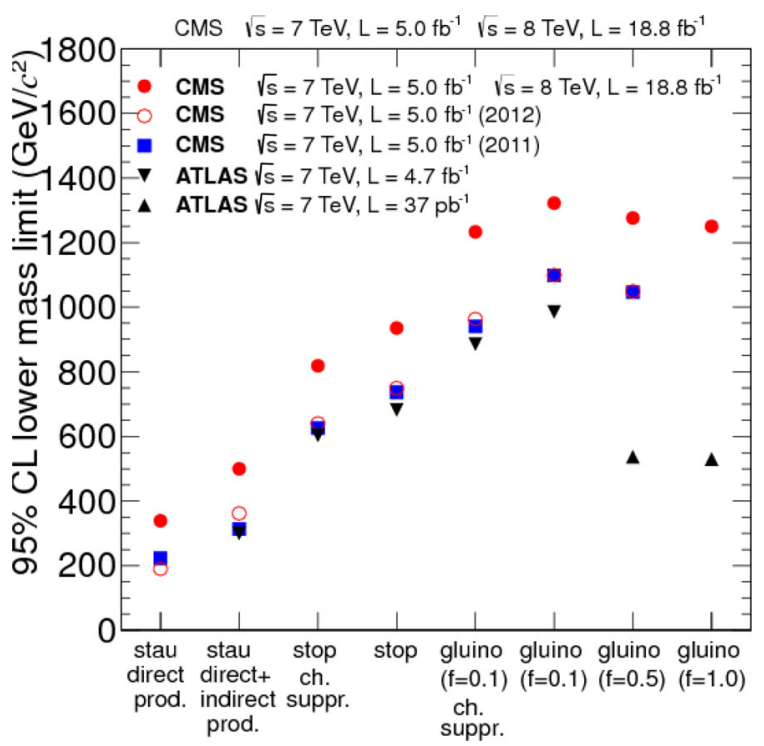

Fig. 38 Lower mass limit at $95 \% \mathrm{CL}$ on different scenarios for longlived particles [125]

surements: $\beta \gamma^{14}$ from the pixel detector by inverting the Bethe-Bloch function, and $\beta$ from the calorimeter and muon spectrometer by measuring the arrival time in these devices. Together with the measure of the particle momentum $p$ in the tracker, or in the muon spectrometer, the composite particle mass $m=p /(\beta \gamma)$ can be inferred.

Events are selected by dedicated slow muon or $E_{\mathrm{T}}^{\text {miss }}$ triggers - the latter case is justified by the modest calorimeter energy depositions of the $R$-hadrons combined with highenergetic jets from initial-state radiation. The background is evaluated by building templates for $p, \beta$, and $\beta \gamma$ in signal depopulated regions (like in Fig. 36, signal regions are generally background free). Non-colored particles can also be detector-stable and behave like heavy muons. Therefore, the analysis techniques are similar to the ones used for colored particles. However, in that case, the best performing signal regions are the ones requiring two detector-stable slepton candidates.

The current mass limits from detector-stable particles are presented in Fig. 38 [125]—note that the ATLAS results are still those from the $7 \mathrm{TeV}$ run [30]. Because no Standard Model backgrounds exist, the limits obtained are generally higher than in the prompt-decay case. This is especially true for top and bottom squarks and staus-where no limits on the direct production exist in the RPC prompt case. The gluino masses below 1.2 TeV are excluded independent of the hypotheses made for the interaction of the $R$-hadrons and $R$-gluino balls.

A fraction of these slow-moving particles may come to rest within the detector volume and only decay later as

$\overline{14}$ The variable $\beta$ is the particle velocity and $\gamma$ is the Lorentz boost.

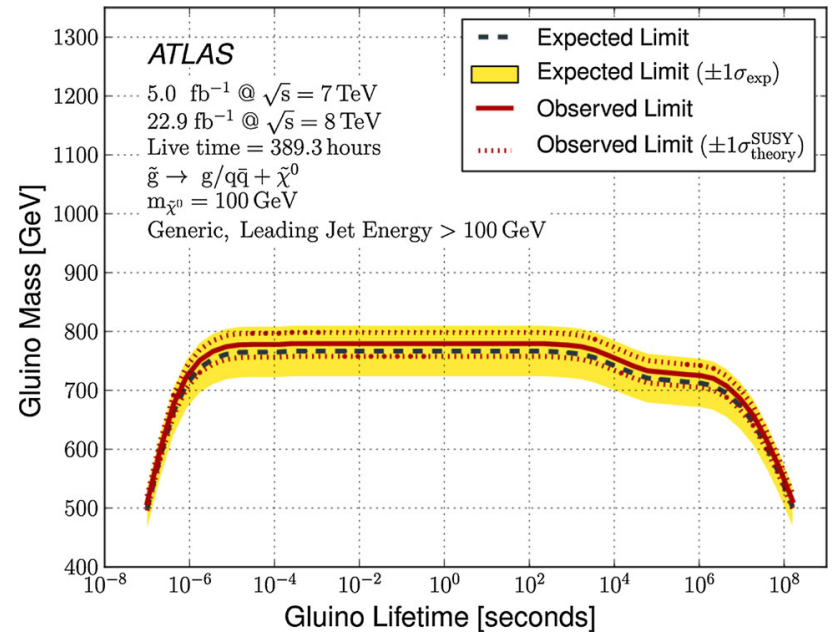

Fig. 39 Exclusion limit at $95 \%$ CL for the stopped gluino searches in mass-lifetime plane of the gluino [126]

$\widetilde{\mathrm{g}} \rightarrow \mathrm{q} \overline{\mathrm{q}} \widetilde{\chi}_{1}^{0}, g \widetilde{\chi}_{1}^{0}$. A particular case is given when this happens in the calorimeter. The signature consists of a highenergetic jet(s) in absence of collisions. The main background is then caused by calorimeter noise bursts, cosmic rays with high energy deposit or beam halo-the leading background. Gluinos below $850 \mathrm{GeV}$ are excluded for a gluino lifetime between $10 \mu$ s and 15 min; see Fig. 39 [126]. This signature is generally present in unnatural SUSY models, where the gluino and the LSP are the only accessible sparticles at the LHC.

\subsection{Beyond MSSM}

The MSSM has firmly been established since 30 years and serves as a basis for most of the SUSY searches at the LHC. However, many possible extensions exist, as shown in Fig. 40. With mild departure from MSSM parsimony, they could explain the current absence of SUSY signals at LHC. In addition, they generally predict new signatures that could be searched for at LHC. We briefly review here the status of these beyond MSSM searches.

The first category of models adds a gauge-singlet superfield to the MSSM. More specifically in the NMSSM [128], two additional Higgs bosons and one neutralino (singlino, $\widetilde{S}$ ) are added to the MSSM. The extra Higgs are searched for as a peak around $10 \mathrm{GeV}$ in the invariant di-muon mass [129]. The naturalness constraints from the $126 \mathrm{GeV}$ Higgs mass are relaxed and singlino-like LSPs with very small couplings are possible-changing the phenomenology of the SUSY EW sector. Because of this addition, these models predict final states with multi-leptons and $E_{\mathrm{T}}^{\text {miss }}$. Even if no dedicated searches exist yet at LHC, reinterpretations of present EWKino searches, presented in Sect. 6, have already started to constrain NMSSM models [130]. Apart from NMSSM, 


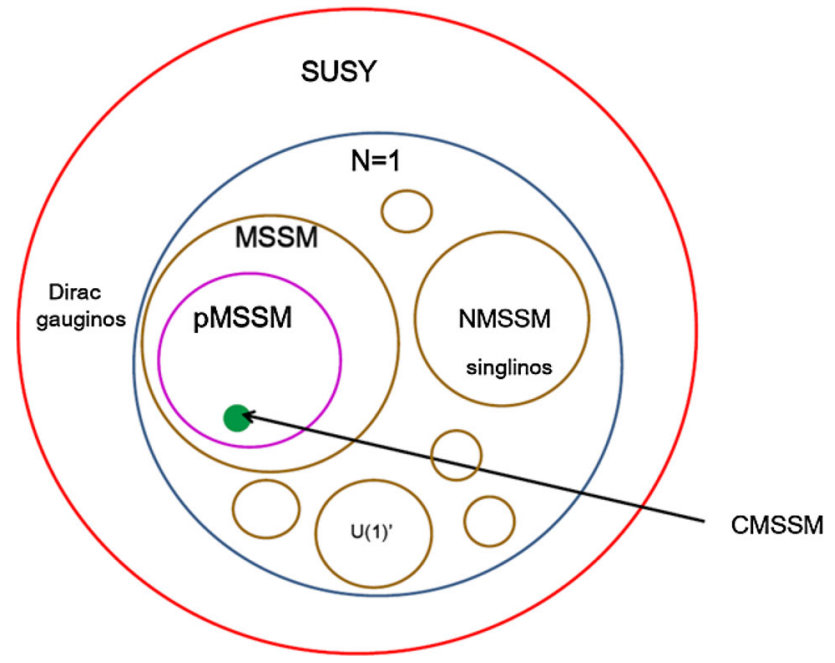

Fig. 40 SUSY phase space and associated theories [127]

another popular model is stealth SUSY [131], where the invisible singlet and singlino are mass degenerate and light, which reduces drastically the amount of $E_{\mathrm{T}}^{\text {miss }}$-all SUSY cascade decays end like $\widetilde{\mathrm{S}} \rightarrow \mathrm{S}(\rightarrow \mathrm{jj}) \widetilde{\mathrm{G}}$ with a poorly boosted $\widetilde{\mathrm{G}}$. Experimental signatures comprise a low $E_{\mathrm{T}}^{\text {miss }}$, displaced vertex and high-multiplicity final states including photons and a high number of b-jets. CMS already places a limit on stealth scenarios where S decays via a photon [132]. Limits of $1.5 \mathrm{TeV}$ on squarks initiating cascade decays are obtained. More refined searches are currently going on.

The second category of models postulates the gluino to be of Dirac type instead of Majorana as in the MSSM. ${ }^{15}$ This happens in theories which extend the $R$-parity concept to a continuous symmetry (MRSSM [133]), hybrid $\mathrm{N}=1 / \mathrm{N}=2$ model [134] and Supersoft SUSY (SSSM [135]). For the two first models, a new particle (the sgluon) completes the MSSM multiplet composed of gluons and gluinos. In all models, the constraint on the gluino mass in the natural spectrum is relaxed since the radiative corrections are truncated. Because of that, gluino-gluino cross sections are expected to be lower than in the MSSM, weakening the current constraint. The sgluon provides also new signatures to search for. Above a mass of $350 \mathrm{GeV}$, sgluons dominantly decay in two tops. They can for example be searched for by asking two same-sign leptons (no public results are presently available). At lower mass, sgluons decay in two gluons, giving a pair of two-jet resonances with equal mass. A unique pairing of the four highest energetic jets is achieved for each event by minimizing the pairwise separation. A peak in the dijet mass distribution is then searched for, while the shape and the normalization of the multi-jet background are esti-

\footnotetext{
${ }^{15}$ It can also be the case for other gauginos but only the gluino is considered since LHC limits are generally quite strong on the gluino mass.
}

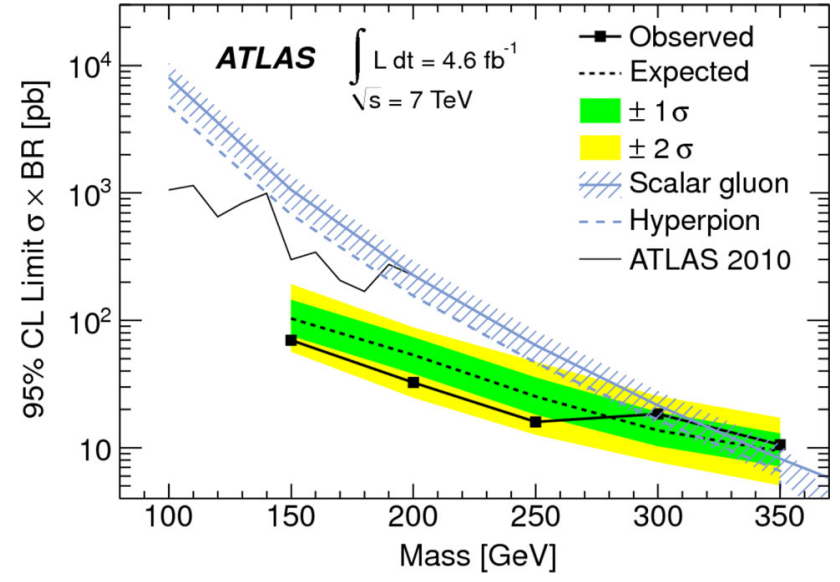

Fig. 41 Exclusion limits at $95 \% \mathrm{CL}$ on the sgluon pair production cross section as a function of the sgluon mass [136]

mated by a data-driven method. Sgluons are excluded for masses below $300 \mathrm{GeV}$; see Fig. 41 [136]. It is interesting to note that this signature, unique in BSM models at LHC, is limited at low mass by the multi-jet energy trigger threshold and therefore strong limits are already obtained with the first run at $7 \mathrm{TeV}$ in 2010.

\section{SUSY prospects at LHC beyond the first run}

The LHC program is approved until 2022. In this program, the center-of-mass energy will be $13 \mathrm{TeV}$ at the restart and it should reach gradually $14 \mathrm{TeV}$. Expected luminosities are $\sim 100 \mathrm{fb}^{-1}$ at the end of 2017 and $\sim 300 \mathrm{fb}^{-1}$ in 2022. A possible extension, called High-Luminosity-LHC (HLLHC), is planned from 2024 to 2030-2035 (Run 3) and could deliver ultimately $\sim 3000 \mathrm{fb}^{-1}$. The average number of pileup events per bunch crossing is expected to rise up to 140 in 2030. Expected discovery sensitivities and exclusion limits have recently been extracted by ATLAS and CMS for benchmark processes of plain vanilla MSSM, i.e. $R$-parity conservation and $\widetilde{\chi}_{1}^{0} \operatorname{LSP}[137,138]$.

With the increase in beam energy in 2015, the strong SUSY cross sections are greatly enhanced, opening a new phase space to explore with already a low integrated luminosity recorded $\left(1-10 \mathrm{fb}^{-1}\right)$. A few examples are given here for sparticles at the energy frontier. The cross section for gluinogluino production (with squarks decoupled) is enhanced by more than a factor of 20 for gluino masses of around $1.3 \mathrm{TeV}$. The cross section for the pair production of squarks of the first/second generation as well as for third-generation squarks rises by about a factor of 10 for squark masses of $800 \mathrm{GeV}$. With $300 \mathrm{fb}^{-1}$, mass degenerate squarks (of the first and second generation) and gluinos of up to $2.7 \mathrm{TeV}$ could be discovered with $5 \sigma$ significance. For higher gluino (squarks) 
masses, e.g. $3.5 \mathrm{TeV}$, squark (gluino) masses could be discovered up to 2.5 (2) TeV. With the HL-LHC, a gain of about $300 \mathrm{GeV}$ in the mass range is expected. Finally, the top squark could be discovered up to masses of about 800 (1000) GeV with $300(3000) \mathrm{fb}^{-1}$, assuming a $100 \%$ branching ratio to a top quark and a $\widetilde{\chi}_{1}^{0}$.

Searches for electroweak particles like neutralinos and charginos benefit more from the large expected integrated luminosity than for the increase in $\sqrt{s}$ due to their low cross sections. By the end of Run 3, neutralinos $\left(\widetilde{\chi}_{2}^{0}\right)$ and charginos $\left(\widetilde{\chi}_{1}^{ \pm}\right)$decaying to $\mathrm{Z} \widetilde{\chi}_{1}^{0}$ and $\mathrm{W} \widetilde{\chi}_{1}^{0}$, respectively, as in scenario (a) of Fig. 2, can be discovered up to a mass of $700 \mathrm{GeV}$ for $\widetilde{\chi}_{1}^{0}$ masses of up to $200 \mathrm{GeV}$ with a $5 \sigma$ significance. In the case of no signal, the exclusion limits are about 200 to $300 \mathrm{GeV}$ higher. Some sensitivity is also expected in the compressed and more natural scenario (c) of Fig. 2 where the $\tilde{\chi}_{1}^{0}$ is higgsino-like.

In summary, the full harvest of the LHC, including the HLLHC, could explore the largest part of the most interesting weak-scale SUSY phase space, which will remain a hot topic to be tested for at least the next two decades.

\section{Conclusions}

ATLAS and CMS, the two general-purpose LHC experiments, have developed a coherent and ambitious program to search for new particles at the energy frontier, $O(0.1-1 \mathrm{TeV})$ with $25 \mathrm{fb}^{-1}$ of proton-proton collision data with center-ofmass energies of 7 and $8 \mathrm{TeV}$. These efforts were successful: a Higgs boson of $126 \mathrm{GeV}$ mass was discovered after two years of running, closing the list of Standard Model particles to be found.

This discovery fits with expectations from the minimal realization of $\mathrm{N}=1$ SUSY, a.k.a. weak-scale SUSY or MSSM. However, this model also predicts new particles at the energy frontier that could solve the gauge hierarchy problem of the Standard Model, i.e. the quadratic divergence of the Higgs mass at higher energy. Assuming that $R$-parity is conserved, the plain vanilla SUSY solution predicts a 'natural' or low fine-tuning mass spectrum composed of gluino masses around $1 \mathrm{TeV}$, top squark and left-handed bottom squark masses around $500 \mathrm{GeV}$, and chargino/neutralino masses below $500 \mathrm{GeV}$. All other SUSY particles could be of much higher mass. In this framework, the two favored LSP candidates could be the lightest neutralino or the gravitino. ATLAS and CMS experiment have probed, by direct searches, the uncharted heart of the MSSM spectrum, attracting high attention from the community. To date, these dedicated searches, mainly based on the presence of multi-jets and $E_{\mathrm{T}}^{\text {miss }}$ (but not only these), have not revealed any sign of new physics.
Not all results from Run 1 are currently available but the following general conclusions could be drawn: the gluino mass, governed by only one SUSY parameter, $M_{3}$, could be excluded below $1 \mathrm{TeV}$ irrespective of the SUSY mass spectrum in between the gluino and the LSP, and the nature of the LSP. This conclusion applies well for open SUSY spectra, i.e. a mass difference between gluino and the LSP above $\mathrm{O}(500 \mathrm{GeV})$. But the excluded gluino mass region should be lowered to $600 \mathrm{GeV}$ when considering more and more compressed spectra, because the jet and $E_{\mathrm{T}}^{\text {miss }}$ softening decreases the acceptance - the presence of isolated lepton(s) can partially correct for that.

Constraints on squarks of the first and second generation are generally softer and less general, since mass degeneracy between families is often assumed. A strong focus was put on the third squark generation (top and bottom squark) because of their particular position in the natural spectrum. When top and bottom squarks are directly produced, final states are generally less complex than for the gluino and squarks of the first and second generation and composed of multiple b-jets and lepton(s). Dedicated searches dramatically shrink the allowed window, but they are (presently) unsuccessful. As an illustration, holes in the top squark searches are presently located near the top mass funnel, $m_{\mathrm{t}}=m_{\mathrm{t}}+m_{\widetilde{\chi}_{1}^{0}}$, for $m_{\widetilde{\chi}_{1}^{0}}>100 \mathrm{GeV}$, at low mass difference between top squark and $\tilde{\chi}_{1}^{0}$ or in very intricate top squark decay chains.

The weak SUSY sector (charginos, neutralinos, and sleptons) is also probed extensively at LHC. Because of the lower cross section, the Standard Model background is only reducible considering multi-lepton final states. These leptons are provided by the leptonic decay of the W, Z, Higgs and/or sleptons. Compared with the other sectors no strong general conclusions are drawn (yet) because of the high number of possible final states and the complexity of the sector governed by around 10 parameters. Nonetheless, constraints are generally always going beyond the LEP ones, and biting the natural spectrum in many cases.

Therefore it is fair to say that even if not all $8 \mathrm{TeV}$ results are currently available, plain vanilla MSSM is under high pressure. More definitive conclusions will come when all these results will be interpreted with a full scan of the main 19-20 MSSM parameters (some assumptions are made on the other 105-19 parameters), which will happen in 2014. Meanwhile, more focus has been put on searches for longlived particles, $R$-parity violating scenarios and new theoretical ideas (Stealth SUSY, Dirac gauginos, extra gauge-singlet superfield) that provide many striking signatures, generally background free and relying more on detector performance. Such scenarios are in most cases compatible with the absence of experimental evidence for plain vanilla MSSM. A huge number of possibilities exist and the most important ones have been covered (or are worked on), with presently no sign 
of SUSY. It is interesting to note that the gluino mass is constrained to be above $1 \mathrm{TeV}$ in the models that have been considered.

All these direct searches for new particles have been complemented by indirect searches, which we briefly mention for completeness. At LHC, the main improvement comes from the measurement of the $\mathrm{B}_{\mathrm{s}}^{0} \rightarrow \mu^{+} \mu^{-}$branching ratio, where the leading SUSY contributions involve SUSY Higgses $\left(\mathrm{A}^{0}\right.$ and $\left.\mathrm{H}^{0}\right)$ in penguin diagrams. Good agreement with the Standard Model was found [139,140], and no irreducible limit exists on SUSY models. Other indirect evidence for new physics could be found when searching for the flavorchanging decay of the top quark like $\mathrm{t} \rightarrow \mathrm{cH}$, which is very strongly suppressed in the Standard Model BR $=\mathrm{O}\left(10^{-15}\right)$. Contributions from SUSY Higgses in virtual loops of the decay amplitude can enhance the cross section significantly, by factors up to nine orders of magnitude. Nevertheless, up to now, no evidence for this decay has been found when considering $\mathrm{H} \rightarrow \mathrm{WW}, \mathrm{ZZ}, \tau \tau$ leptonic decay [69] and $\mathrm{H} \rightarrow \gamma \gamma$ decay [141].

A new phase of exploration will be opened in 2015 with the restart of the LHC at higher energy. But the present situation after the first run could well fit an Ernest Rutherford quote: 'An alleged scientific discovery has no merit unless it can be explained to a barmaid'. We can now happily discuss with the barmaid of the SM bar, but we could not yet find the door of the SUSY bar!

Acknowledgments We would like to thank our colleagues in CMS and ATLAS who helped us producing the combined plots.

Open Access This article is distributed under the terms of the Creative Commons Attribution License which permits any use, distribution, and reproduction in any medium, provided the original author(s) and the source are credited.

Funded by $\mathrm{SCOAP}^{3}$ / License Version CC BY 4.0.

\section{References}

1. L. Evans, P. Briant, JINST 3, S08001 (2008)

2. ATLAS Collaboration, JINST 3, S08003 (2008)

3. CMS Collaboration, JINST 3, S08004 (2008)

4. ATLAS Collaboration, Phys. Lett. B 716, 1 (2012)

5. CMS Collaboration, Phys. Lett. B 716, 30 (2012)

6. P. Ramond, SUSY: the early years (1966-1976). Eur. Phys. J. C 74, 2698 (2014). doi:10.1140/epjc/s10052-013-2698-x. arXiv: 1401.5977

7. P. Fayet, SUSY: From 1976 to the LEP years (2014)

8. S.P. Martin, A Supersymmetry Primer (2011). arXiv:hep-ph/ 9709356

9. A. Djouadi, Implications of the Higgs discovery for the MSSM. Eur. Phys. J. C 74, 2704 (2014). doi:10.1140/epjc/ s10052-013-2704-3. arXiv:1311.0720

10. S.P. Martin, P. Ramond, Phys. Rev. D 48, 5365 (1993)

11. G.G. Ross, SUSY: Quo Vadis?. Eur. Phys. J. C 74, 2699 (2014). doi:10.1140/epjc/s10052-013-2699-9

12. Y. Kats, P. Meade, M. Reece, D. Shih, JHEP 1202, 115 (2012)
13. L. Hall, Searches for SUSY at the LHC. LBL Workshop, 19-21 Oct 2011 (2011)

14. M. Kramer, A. Kulesza, R. Van der Leeuw, M. Mangano, S. Padhi, T. Plehn, X. Portell, Supersymmetry production cross sections in pp collisions at $\sqrt{s}=7 \mathrm{TeV}$ (2012). arXiv:1206.2892

15. W. Beenakker, R. Hopker, M. Spira, P.M. Zerwas, Nucl. Phys. B 492, 51 (1997)

16. A. Kulesza, L. Motyka, Phys. Rev. Lett. 102, 111802 (2009)

17. A. Kulesza, L. Motyka, Phys. Rev. D 80, 095004 (2009)

18. W. Beenakker, S. Brensing, M. Kramer, A. Kulesza, E. Laenen et al., JHEP 0912, 041 (2009)

19. W. Beenakker, S. Brensing, M. Kramer, A. Kulesza, E. Laenen et al., Int. J. Mod. Phys. A 26, 2637 (2011)

20. The Royal Swedish Academy of Sciences. Press release of the 2013 Physics Nobel Prize (2013)

21. D. Froidevaux, P. Sphicas, Ann. Rev. Nucl. Part. Sci. 56, 375 (2006)

22. CMS Collaboration, Commissioning of the particle-flow reconstruction in minimum-bias and jet events from pp collisions at 7 TeV. CMS-PAS-PFT-10-002 (2010)

23. M. Cacciari, G.P. Salam, G. Soyez, JHEP 0804, 063 (2008)

24. M. Cacciari, G.P. Salam, Phys. Lett. B 641, 57 (2006)

25. CMS Collaboration, JINST 6, P11002 (2011)

26. ATLAS Collaboration, Eur. Phys. J. C 73, 2304 (2013)

27. ATLAS Collaboration, Commissioning of the ATLAS highperformance b-tagging algorithms in the $7 \mathrm{TeV}$ collision data. ATLAS-CONF-2011-102 (2011)

28. CMS Collaboration, JINST 8, P04013 (2013)

29. CMS Collaboration, JINST 7, P01001 (2012)

30. ATLAS Collaboration, Phys. Lett. B 720, 277 (2013)

31. I. Hinchliffe, F.E. Paige, M. Shapiro, J. Soderqvist, W. Yao, Phys. Rev. D 55, 5520 (1997)

32. D. Tovey, Phys. Lett. B 498, 1 (2001)

33. ATLAS Collaboration, Phys. Rev. Lett. 106, 131802 (2011)

34. L. Randall, D. Tucker-Smith, Phys. Rev. Lett. 101, 221803 (2008)

35. C. Rogan, Kinematics for new dynamics at the LHC (2010). arXiv: 1006.2727

36. C. Lester, D. Summers, Phys. Lett. B 463, 99 (1999)

37. A. Barr, C. Lester, P. Stephens, J. Phys. G 29, 2343 (2003)

38. M. Burns, K. Kong, K.T. Matchev, M. Park, JHEP 0903, 143 (2009)

39. D. Tovey, JHEP 0804, 034 (2008)

40. G. Polesello, D. Tovey, JHEP 1003, 030 (2010)

41. A.J. Barr, T.J. Khoo, P. Konar, K. Kong, C.G. Lester, K.T. Matchev, M. Park, Phys. Rev. D 84, 095031 (2011)

42. ATLAS Collaboration, Phys. Rev. D 87, 012008 (2013)

43. M.L. Mangano, M. Moretti, F. Piccinini, R. Pittau, A.D. Polosa, JHEP 0307, 001 (2003)

44. T. Gleisberg, S. Hoeche, F. Krauss, M. Schonherr, S. Schumann et al., JHEP 0902, 007 (2009)

45. J. Alwall, M. Herquet, F. Maltoni, O. Mattelaer, T. Stelzer, JHEP 1106, 128 (2011)

46. S. Frixione, F. Stoeckli, P. Torrielli, B.R. Webber, C.D. White, The MCaNLO 4.0 Event Generator (2010). arXiv:1010.0819

47. P. Nason, JHEP 0411, 040 (2004)

48. S. Frixione, P. Nason, C. Oleari, JHEP 0711, 070 (2007)

49. S. Alioli, P. Nason, C. Oleari, E. Re, JHEP 1006, 043 (2010)

50. M. Bahr, S. Gieseke, M. Gigg, D. Grellscheid, K. Hamilton et al., Eur. Phys. J. C 58, 639 (2008)

51. T. Sjöstrand, S. Mrenna, P.Z. Skands, JHEP 05, 026 (2006)

52. GEANT4 Collaboration, Nucl. Instrum. Methods A 506, 250 (2003)

53. S. Abdullin, P. Azzi, F. Beaudette, P. Janot, A. Perrotta, in CHEP 2011. J. Phys.: Conference Series, vol. 331, p. 032049 (2010) 
54. ATLAS Collaboration, The simulation principle and performance of the ATLAS fast calorimeter simulation FastCaloSim. ATLPHYS-PUB-2010-013 (2010)

55. A. Read, J. Phys. G: Nucl. Part. Phys. 28, 2693 (2002)

56. A.H. Chamseddine, R.L. Arnowitt, P. Nath, Phys. Rev. Lett. 49, 970 (1982)

57. R. Barbieri, S. Ferrara, C.A. Savoy, Phys. Lett. B 119, 343 (1982)

58. L.J. Hall, J.D. Lykken, S. Weinberg, Phys. Rev. D 27, 2359 (1983)

59. R. Arnowitt, P. Nath, Phys. Rev. Lett. 69, 725 (1992)

60. G.L. Kane, C.F. Kolda, L. Roszkowski, J.D. Wells, Phys. Rev. D 49, 6173 (1994)

61. M. Dine, A.E. Nelson, Y. Nir, Y. Shirman, Phys. Rev. D 53, 2658 (1996)

62. L. Randall, R. Sundrum, Nucl. Phys. B 557, 79 (1999)

63. G.F. Giudice, M.A. Luty, H. Murayama, R. Rattazzi, JHEP 9812 , 027 (1998)

64. N. Arkani-Hamed, P. Schuster, N. Toro, J. Thaler, L.-T. Wang et al., MARMOSET: The path from LHC data to the new standard model via on-shell effective theories (2007). arXiv:hep-ph/0703088

65. J. Alwall, P. Schuster, N. Toro, Phys. Rev. D 79, 075020 (2009)

66. D. Alves et al., J. Phys. G 39, 105005 (2012)

67. A. Djouadi et al., The minimal supersymmetric standard model: group summary report (1998). arXiv:hep-ph/9901246

68. CMS Collaboration, Phys. Lett. B 725, 243 (2013)

69. CMS Collaboration, Search for new physics in multijets and missing momentum final state in proton-proton collisions at $\sqrt{s}=8$ TeV. JHEP (2014) (Accepted). arXiv:1402.4770

70. ATLAS Collaboration, JHEP 1310, 130 (2013)

71. CMS Collaboration, Eur. Phys. J. C 73, 2568 (2013)

72. ATLAS Collaboration, Phys. Rev. D 86, 092002 (2012)

73. CMS Collaboration, Phys. Lett. B. (2013) (Submitted). arXiv: 1311.4937

74. CMS Collaboration, JHEP 1401, 163 (2014). arXiv:1311.6736

75. A. Arvanitaki, M. Baryakhtar, X. Huang, K. Van Tilburg, G. Villadoro, JHEP 1403, 022 (2014). arXiv: 1309.3568

76. ATLAS Collaboration, Phys. Lett. B 718, 411 (2012)

77. CMS Collaboration, JHEP 1303, 111 (2013)

78. ATLAS Collaboration, Phys. Lett. B 719, 261 (2013)

79. ATLAS Collaboration, Eur. Phys. J. C 72, 2215 (2012)

80. CMS Collaboration, Eur. Phys. J. C 73, 2493 (2013)

81. CMS Collaboration, Search for anomalous production of events with three or more leptons in pp collisions at $\sqrt{s}=8 \mathrm{TeV}$. Phys. Rev. D (2014) (Submitted). arXiv:1404.5801

82. ATLAS Collaboration, Search for direct top squark pair production in events with a $\mathrm{Z}$ boson, b-jets and missing transverse momentum in pp collisions at $\sqrt{s}=8 \mathrm{TeV}$ with the ATLAS detector. Eur. Phys. J. C (2014) (Submitted). arXiv:1403.5222

83. CMS Collaboration, Eur. Phys. J. C 73, 2677 (2013)

84. Y. Bai, H. Cheng, J. Gallicchio, J. Gu, JHEP 1207, 110 (2012)

85. ATLAS Collaboration, Search for direct top-squark pair production in final states with two leptons in pp collisions at $\sqrt{s}=8$ $\mathrm{TeV}$ with the ATLAS detector. Eur. Phys. J. C (2014) (Submitted). arXiv: 1403.4853

86. ATLAS Collaboration, JHEP 1310, 189 (2013)

87. CMS Collaboration, Phys. Rev. Lett. 112, 161802 (2014). arXiv: 1312.3310

88. ATLAS Collaboration, Phys. Rev. D 89, 032002 (2014). arXiv: 1312.1956

89. ATLAS Collaboration. JHEP 1404, 169 (2014). arXiv:1402.7029

90. CMS Collaboration, JHEP 1209, 094 (2012)

91. ATLAS Collaboration, JHEP 1304, 075 (2013)

92. CMS Collaboration, JHEP 1211, 147 (2012)
93. ATLAS Collaboration, Search for direct production of charginos, neutralinos and sleptons in final states with two leptons and missing transverse momentum in pp collisions at $\sqrt{s}=8$ $\mathrm{TeV}$ with the ATLAS detector JHEP (2013). (Submitted). arXiv: 1403.5294

94. R. Barbier, C. Berat, M. Besancon, M. Chemtob, A. Deandrea et al., Phys. Rep. 420, 1 (2005)

95. ATLAS Collaboration, JHEP 1212, 124 (2012)

96. K. Desch, S. Fleischmann, P. Wienemann, H. Dreiner, S. Grab, Phys. Rev. D 83, 015013 (2011)

97. CMS Collaboration, JHEP 1206, 169 (2012)

98. CMS Collaboration, Phys. Rev. Lett. 111, 221801 (2013)

99. ATLAS Collaboration, Phys. Lett. B 723, 15 (2013)

100. M. Chemtob, Prog. Part. Nucl. Phys. 54, 71 (2005)

101. ZEUS Collaboration, Phys. Rev. D 86, 012005 (2012). arXiv: 1205.5179

102. H1 Collaboration, Phys. Lett. B 704, 388 (2011). arXiv: 1107.3716

103. CMS Collaboration, Phys. Rev. Lett. 110, 081801 (2013)

104. R.S. Chivukula, M. Golden, E.H. Simmons, Phys. Lett. B 257, $403(1991)$

105. R.S. Chivukula, M. Golden, E.H. Simmons, Nucl. Phys. B 363, 83 (1991)

106. CMS Collaboration, Phys. Lett. B 730, 193 (2014). arXiv: 1311.1799

107. CDF Collaboration, Phys. Rev. Lett. 107, 042001 (2011)

108. CMS Collaboration, Phys. Rev. Lett. 107, 101801 (2011)

109. ATLAS Collaboration, JHEP 1212, 086 (2012)

110. S. Raby, Phys. Rev. D 56, 2852 (1997)

111. H. Baer, K. Cheung, J.F. Gunion, Phys. Rev. D 59, 075002 (1999). arXiv:hep-ph/9806361

112. A. Mafi, S. Raby, Phys. Rev. D 63, 055010 (2001)

113. S. Raby, Phys. Lett. B 422, 158 (1998)

114. A. Mafi, S. Raby, Phys. Rev. D 62, 035003 (2000)

115. CMS Collaboration, JHEP 1211, 172 (2012)

116. ATLAS Collaboration, Phys. Rev. D 88, 012001 (2013)

117. CMS Collaboration, Phys. Lett. B 722, 273 (2013)

118. ATLAS Collaboration, Phys. Lett. B 719, 280 (2013)

119. CMS Collaboration, JHEP 1302, 085 (2013)

120. ATLAS Collaboration, Phys. Rev. D 88, 112006 (2013)

121. L.J. Hall, Y. Nomura, S. Shirai, JHEP 1301, 036 (2013)

122. A. Arvanitaki, N. Craig, S. Dimopoulos, G. Villadoro, JHEP 1302, $126(2013)$

123. N. Arkani-Hamed, A. Gupta, D.E. Kaplan, N. Weiner, T. Zorawski, Simply Unnatural Supersymmetry (2012). arXiv:1212.6971

124. M. Ibe, S. Matsumoto, T.T. Yanagida, Phys. Rev. D 85, 095011 (2012)

125. CMS Collaboration, JHEP 1307, 122 (2013)

126. ATLAS Collaboration, Phys. Rev. D 88, 112003 (2013)

127. T. Rizzo, The BSM Zoo. 40th SLAC Summer Institute (2012)

128. U. Ellwanger, C. Hugonie, A.M. Teixeira, Phys. Rept. 496, 1 (2010)

129. CMS Collaboration, Phys. Rev. Lett. 109, 121801 (2012)

130. U. Ellwanger, JHEP 1311, 108 (2013)

131. J. Fan, M. Reece, T. Ruderman, JHEP 1111, 012 (2012)

132. CMS Collaboration, Phys. Lett. B 719, 42 (2013)

133. G.D. Kribs, E. Poppitz, N. Weiner, Phys. Rev. D 78, 055010 (2008)

134. S.Y. Choi, M. Drees, A. Freitas, P.M. Zerwas, Phys. Rev. D 78, 095007 (2008)

135. G.D. Kribs, A. Martin, Phys. Rev. D 85, 115014 (2012)

136. ATLAS Collaboration, Eur. Phys. J. C 73, 2263 (2013)

137. Y. Gershtein, M. Luty, M. Narain, L.T. Wang, D. Whiteson et al., New Particles Working Group Report of the Snowmass 2013 Community Summer Study (2013). arXiv:1311.0299 
138. D. Abbaneo et al., ECFA High Luminosity LHC Experiments Workshop: Physics and Technology Challenges. ECFA-13-284 (2013)

139. LHCb Collaboration, Phys. Rev. Lett. 110, 021801 (2013)
140. CMS Collaboration, Phys. Rev. Lett. 111, 101804 (2013)

141. ATLAS Collaboration, Search for top quark decays $\mathrm{t} \rightarrow \mathrm{q} H$ with $\mathrm{H} \rightarrow \gamma \gamma$ using the ATLAS detector, JHEP (2014) (Submitted). arXiv: 1403.6293 